

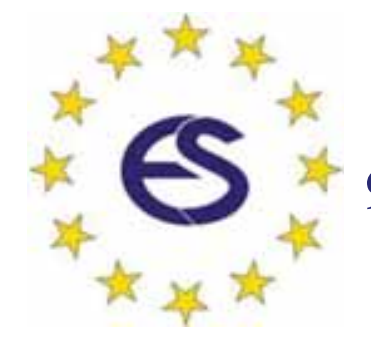

\section{EUROSIM 2019 \\ $9^{\text {th }}$ EUROSIM Congress on Modelling and Simulation \\ La Rioja, Logroño, Spain, July 2019}
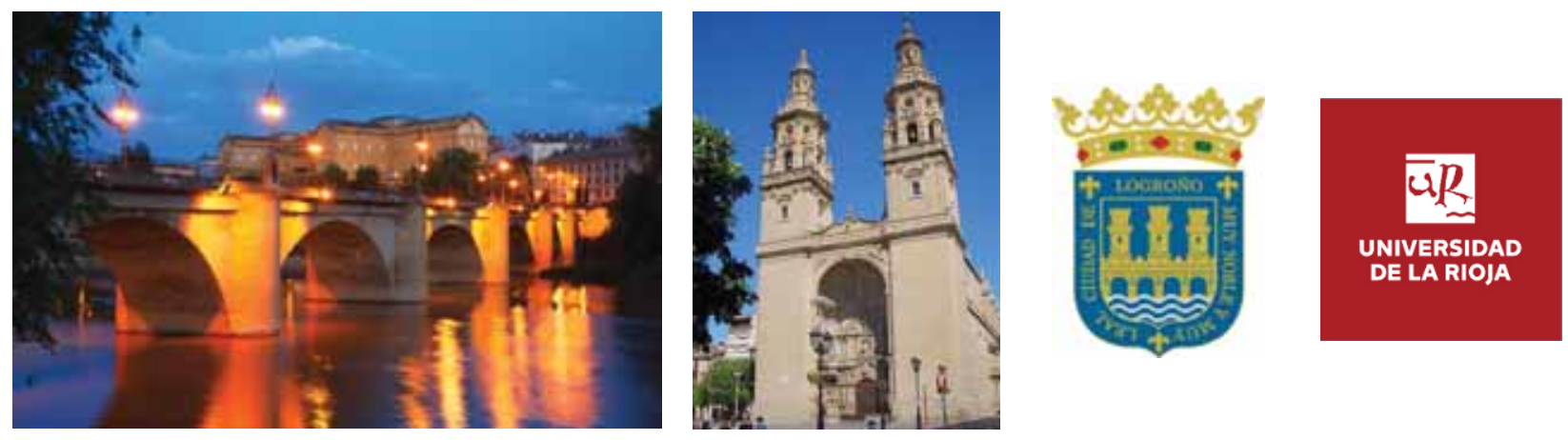

EUROSIM Congresses are the most important modelling and simulation events in Europe. For EUROSIM 2019, we are soliciting original submissions describing novel research and developments in the following (and related) areas of interest: Continuous, discrete (event) and hybrid modelling, simulation, identification and optimization approaches. Two basic contribution motivations are expected: M\&S Methods and Technologies and M\&S Applications. Contributions from both technical and non-technical areas are welcome.

Congress Topics The EUROSIM 2019 Congress will include invited talks, parallel, special and poster sessions, exhibition and versatile technical and social tours. The Congress topics of interest include, but are not limited to:

Intelligent Systems and Applications

Hybrid and Soft Computing

Data \& Semantic Mining

Neural Networks, Fuzzy Systems \&

Evolutionary Computation

Image, Speech \& Signal Processing

Systems Intelligence and

Intelligence Systems

Autonomous Systems

Energy and Power Systems

Mining and Metal Industry

Forest Industry

Buildings and Construction

Communication Systems

Circuits, Sensors and Devices

Security Modelling and Simulation
Bioinformatics, Medicine, Pharmacy and Bioengineering

Water and Wastewater Treatment,

Sludge Management and Biogas Production

Condition monitoring, Mechatronics and maintenance

Automotive applications

e-Science and e-Systems

Industry, Business, Management, Human Factors and Social Issues

Virtual Reality, Visualization, Computer Art and Games

Internet Modelling, Semantic Web and Ontologies

Computational Finance \& Economics
Simulation Methodologies and Tools

Parallel and Distributed

Architectures and Systems

Operations Research

Discrete Event Systems

Manufacturing and Workflows

Adaptive Dynamic Programming and Reinforcement Learning

Mobile/Ad hoc wireless networks, mobicast, sensor placement, target tracking

Control of Intelligent Systems

Robotics, Cybernetics, Control Engineering, \& Manufacturing

Transport, Logistics, Harbour, Shipping and Marine Simulation

Congress Venue / Social Events The Congress will be held in the City of Logroño, Capital of La Rioja, Northern Spain. The main venue and the exhibition site is the University of La Rioja (UR), located on a modern campus in Logroño, capital of La Rioja, where 7500 students are registered. The UR is the only University in this small, quiet region in Northern Spain. La Rioja is where the Monasteries of San Millán de la Cogolla, cradle of the first words written in the Spanish language, are situated, sites included in UNESCO's World Heritage List in 1996. Of course, social events will reflect this heritage - and the famous wines in la Rioja.

Congress Team: The Congress is organised by CAE CAE-SMSG, the Spanish simulation society, and Universidad de la Rioja.

Info: Emilio Jiménez, EUROSIM President, emilio.jimenez@unirioja.es Juan Ignacio Latorre, juanignacio.latorre@unavarra.es 


\section{Editorial}

Dear Readers - Is the title page of this issue - two animations from pathways of refugees and migrants in Europe - reality or fiction? 'Reality and fiction', answers this issue's contribution 'Pathways of Migrants and Refugees - a Simulation Approach', and the authors hope 'Models are in any case a simplification of reality, but they should help in better understanding of complex dynamics as migration movement, and the intention of this model is to show strategies to improve the situation of migrant population and resident populations under appropriate prerequisites'. This issue's contribution 'An Overview of Modelling and Simulation Methods for Complex Dynamic Systems' raises the question 'Are we happy with today's mathematical modelling and simulation approaches', and answers critically 'If yes, why we keep failing to solve the basic problems of our societies?'. A third contribution asks directly in its title 'Microscopic Pendulum Modelling: Absurd Idea or Innovative View on Old Problems?' and astonishes with the imagination, that forward agents, backward agents, and lazy agents sit on the pendulum and let it swing.

Is SNE 27(4) an issue with 'unusual' contributions? No, SNE 27(4) publishes contributions which underline the broadness of modelling and simulation, and which put emphasis also on methodology in modelling and simulation - SNE's together sharpened and broadened publication strategy. The readers will find methodology for applications and in applications as well in the articles 'A Framework for the Metamodeling of Multi-variant Systems and Reactive Simulation Model Generation and Execution', 'Planning Future Health: Developing Big Data and System Modelling Pipelines for Health System Research' and in the short note 'How Modelling and Simulation Can Benefit the Most from Machine Learning'. The contribution 'Spectral Analysis of Unevenly Spaced Data: Models and Application in Heart Rate Variability' seems to contrast to the other contributions, but it is as well of methodical nature.

I would like to thank all authors for their contributions to SNE 27(4), especially also for the open and partly unusual formulations, which hopefully initiate new discussions - and other'unusual' contributions. And last but not least thanks to the SNE Editorial Office for layout, typesetting, preparations for printing, with compliments for the 'unusual' proof reading challenge.

Felix Breitenecker, SNE Editor-in-Chief, eic@sne-journal.org; felix.breitenecker@tuwien.ac.at

\section{Contents SNE 27(4)}

Online SNE 27(4), DOI 10.11128/ sne.27.4.1039, ARGESIM Publ. Print SNE 27(4) ISBN 978-3-903024-62-5,

Print-on-Demand, TU Verlag Vienna, www.tuverlag.at

A Framework for the Metamodeling of Multi-variant Systems and Reactive Simulation Model Generation and Execution H. Folkerts, .T Pawletta, C. Deatcu, U. Durak

Microscopic Pendulum Modelling: Absurd Idea or Innovative View on Old Problems? M. Bicher, N. Popper

Spectral Analysis of Unevenly Spaced Data: Models and Application in Heart Rate Variability. M. Bachler

Pathways of Migrants and Refugees - a Simulation

Approach.

F. Breitenecker, T. Vobruba, A. Körner, N. Popper

Planning Future Health: Developing Big Data and System

Modelling Pipelines for Health System Research.

N. Popper, F. Endel, R. Mayer, M. Bicher, B. Glock .... 203

How Modelling and Simulation Can Benefit the Most from Machine Learning. D. Brunmeir, M. Wastian

An Overview of Modelling and Simulation Methods

for Complex Dynamic Systems. P. Groumpos

EUROSIM Societies Short Info N1 - N8 News EUROSIM / EUROSIM Societies N9 - N14

\section{SNE Contact \& Info}

$\rightarrow$ www.sne-journal.org

奉=” office@sne-journal.org, eic@sne-journal.org

$\bowtie$ SNE Editorial Office, Andreas Körner

ARGESIM / M ath. M odelling \& Simulation Group,

Vienna Univ. of Technology / 101,

Wiedner Haupstrasse 8-10, 1040 Vienna , Austria

\section{SNE Simulation Notes Europe}

SNE Online ISSN 2306-0271, SNE Print ISSN 2305-9974

WEB: $\rightarrow$ www.sne-journal.org, DOI prefix 10.11128/sne

Scope: Developments and trends in modelling and simulation in various areas and in application and theory; comparative studies and benchmarks (documentation of ARGESIM Benchmarks on modelling approaches and simulation implementations); modelling and simulation in and for education, simulation-based e-learning; society information and membership information for EUROSIM members (Federation of European Simulation Societies and Groups).

Editor-in-Chief: Felix Breitenecker, TU Wien, Int. f. Analysis and Scientific Computing, Math. M odelling and Simulation Group 莑: Felix.Breitenecker@tuwien.ac.at, 夆= eic@sne-journal.org Layout / Administration: A. Körner, A. Mathe, J. Tanzler, R. Leskovar, et al.; $\equiv$ ": office@sne-journal.org

Print SNE and Print-on-Demand: Grafisches Zentrum and TU-Verlag, TU Wien, Wiedner Hauptstrasse 8-10, 1040, Vienna, Austria www.tuverlag.at

Publisher: ARGESIM ARBEITSGem EINSCHAft Sim ULATION NeWS C/O M ath. Modelling and Simulation Group, TU Wien/101, Wiedner Hauptstrasse 8-10, 1040 Vienna, Austria; www.argesim.org, $\neq=$ info@ @argesim.org on behalf of ASIM www.asim-gi.org and EUROSIM $\rightarrow$ www.eurosim.info (c) ARGESIM / EUROSIM / ASIM 2017 


\section{SNE - Aims and Scope}

Simulation Notes Europe (SNE) provides an international, high-quality forum for presentation of new ideas and approaches in simulation - from modelling to experiment analysis, from implementation to verification, from validation to identification, from numerics to visualisation - in context of the simulation process.

SNE seeks to serve scientists, researchers, developers and users of the simulation process across a variety of theoretical and applied fields in pursuit of novel ideas in simulation and to enable the exchange of experience and knowledge through descriptions of specific applications. SNE follows the recent developments and trends of modelling and simulation in new and/or joining application areas, as complex systems and big data. SNE puts special emphasis on the overall view in simulation, and on comparative investigations, as benchmarks and comparisons in methodology and application. For this purpose, SNE documents the ARGESIM Benchmarks on Modelling Approaches and Simulation Implementations with publication of definitions, solutions and discussions. SNE welcomes also contributions in education in/for/with simulation.

A News Section in SNE provides information for EUROSIM Simulation Societies and Simulation Groups.

SNE, primarily an electronic journal, follows an open access strategy, with free download in basic layout. SNE is the official membership journal of EUROSIM, the Federation of European Simulation Societies and Simulation Groups www.eurosim.info. Members of EUROSIM societies are entitled to download SNE in an elaborate and extended layout, and to access additional sources of benchmark publications, model sources, etc. Print SNE is available for specific groups of EUROSIM societies, and starting with Volume 27 (2017) as printon-demand from TU Verlag, TU Wien. SNE is DOI indexed by CrossRef, identified by DOI prefix 10.11128, assigned to the SNE publisher ARGESIM (www.argesim.org).

Author's Info. Individual submissions of scientific papers are welcome, as well as post-conference publications of contributions from conferences of EUROSIM societies. SNE welcomes special issues, either dedicated to special areas and/or new developments, or on occasion of events as conferences and workshops with special emphasis.

Authors are invited to submit contributions which have not been published and have not being considered for publication elsewhere to the SNE Editorial Office.

SNE distinguishes different types of contributions (Notes), i.e.

- TN Technical Note, 6-10 p. - EN Education Note -6-8 p.

- PN Project Note 6-8 p. - SN Short Note, max. 6 p.

- SW Software Note, 4-6 p. - BN Benchmark Note, 4-8 p.

- ON Overview Note $O N$ - only upon invitation, up to $14 \mathrm{p}$.

Further info and templates (doc, tex) at SNE‘s website. uww.sne-joumal.org

\section{SNE Editorial Board}

SNE - Simulation Notes Europe is advised and supervised by an international scientific editorial board. This board is taking care on peer reviewing of submission to SNE. At present, the board is increasing (see website www.sne-journal.org):

David Al-Dabass, david.al-dabass@ntu.ac.uk, Nottingham Trent University, UK

Felix Breitenecker, Felix.Breitenecker@tuwien.ac.at Vienna Univ. of Technology, Austria, Editor-in-chief

Maja Atanasijevic-Kunc,maja.atanasijevic@fe.uni-lj.si Univ. of Ljubljana, Lab. Modelling \& Control, Slovenia

Aleš Belič, ales.belic@sandoz.com Sandoz / National Inst. f. Chemistry, Slovenia

Peter Breedveld, P.C.Breedveld@el.utwente.nl University of Twenty, Netherlands

Agostino Bruzzone,agostino@itim.unige.it Universita degli Studi di Genova, Italy

Francois Cellier,fcellier@inf.ethz.ch ETH Zurich, Switzerland

Vlatko Čerić,vceric@efzg.hr Univ. Zagreb, Croatia

Russell Cheng,rchc@maths.soton.ac.uk University of Southampton, UK

Roberto Cianci,cianci@dime.unige.it, Math. Eng. and Simulation, Univ. Genova, Italy

Eric Dahlquist,erik.dahlquist@mdh.se, Mälardalen Univ., Sweden

Horst Ecker,Horst.Ecker@tuwien.ac.at Vienna Univ. of Technology, Inst. f. Mechanics, Austria

Vadim Engelson, vadim.engelson@mathcore.com MathCore Engineering, Linköping, Sweden

Edmond Hajrizi, ehajrizi@ubt-uni.net University for Business and Technology, Pristina, Kosovo

András Jávor, javor@eik.bme.hu, Budapest Univ. of Technology and Economics, Hungary

EskoJuuso,esko.juuso@oulu.fi Univ. Oulu, Dept. Process/Environmental Eng., Finland

Kaj Juslin, kaj.juslin@vtt.fi VTT Technical Research Centre of Finland, Finland

Andreas Körner, andreas.koerner@tuwien.ac.at Technical Univ. Vienna, E-Learning Dpt., Vienna, Austria

Francesco Longo,f.longo@unical.it Univ. of Calabria, Mechanical Department, Italy

Yuri Merkuryev, merkur@itl.rtu.lv, Riga Technical Univ.

David Murray-Smith,d.murray-smith@elec.gla.ac.uk University of Glasgow, Fac. Electrical Engineering, UK

Gasper Music,gasper.music@fe.uni-lj.si Univ. of Ljubljana, Fac. Electrical Engineering, Slovenia

Thorsten Pawletta,pawel@mb.hs-wismar.de Univ. Wismar, Dept. Comp. Engineering, Wismar, Germany

Niki Popper,niki.popper@dwh.at dwh Simulation Services, Vienna, Austria

Kozeta Sevrani, kozeta.sevrani@unitir.edu.al Univ. Tirana, Inst.f. Statistics, Albania

Thomas Schriber,schriber@umich.edu University of Michigan, Business School, USA

Yuri Senichenkov, sneyb@dcn.infos.ru St. Petersburg Technical University, Russia

Oliver Ullrich,oullrich@cs.1u.edu Florida International University, USA

Siegfried Wassertheurer, Siegfried.Wassertheurer@ait.ac.at AIT Austrian Inst. of Technology, Vienna, Austria

Sigrid Wenzel, S.Wenzel@uni-kassel.de Univ. Kassel, Inst. f. Production Technique, Germany 


\title{
Variability Modeling for Engineering Applications
}

\author{
Hendrik Folkerts ${ }^{1 *}$, Thorsten Pawletta ${ }^{1}$, Christina Deatcu ${ }^{1}$, Umut Durak ${ }^{2}$ \\ ${ }^{1}$ Research Group Computational Engineering and Automation, University of Applied Sciences Wismar \\ Philipp-M üller-Straße 14, 23966 Wismar, Germany; *hendrik.folkerts@cea-wismar.de \\ ${ }^{2}$ German Aerospace Center, Lilienthalplatz 7, 38108 Braunschweig, Germany
}

SNE 27(4), 2017, 167 - 176, DOI: 10.11128/sne.27.tn.10391 Received: December 10, 2017 (Selected ASIM GMMS/STS 2018 Conf. Publ.), Accepted: December 20, 2017 SNE - Simulation Notes Europe, ARGESIM Publisher Vienna, ISSN Print 2305-9974, Online 2306-0271, www.sne-journal.org

Abstract. The System Entity Structure (SES) is a high level approach for variability modeling, particularly in simulation engineering. The SES is under continuous development. In this context, an enhanced framework is introduced that supports dynamic variability evolution using the SES approach and connects the SES to a model base (MB). Using this framework allows building executable models. However, the main focus of this paper is to show how to use the SES to model complex engineering system configurations using a test bench for valves. Specifying the SES, it is clarified, that the system configuration can be decomposed into generalized design patterns. The design patterns were identified during the development of our MATLAB-based SES toolbox for construction and pruning of SES trees and were employed for testing and verification of the respective functionality.

\section{Introduction}

Variability modeling is an approach to describe more than one system configuration derived from one underlying configuration. The basic system configuration is often refered to as product line [1]. According to [2] the most popular technique of modeling commonality and variability of products in software engineering is feature modeling. Feature models were introduced by Kang in [3], were subsequently extended, and used in various ways.

An important further development of variability modeling has been the notion of variability in time, known as binding time in product line engineering [4]. Hence, variability can now be realized from design time to runtime.
An approach to variability modeling in simulation engineering is the System Entity Structure (SES) introduced by Zeigler in [5]. The objective was to describe a set of system configurations for a family of systems. In the early nineties the idea of combining the SES with a model base (MB) in order to generate an executable model led to the SES/MB approach [6].

An SES is represented by a tree structure, which describes a set of modular, hierarchical system structures, defines references to basic models in a MB and specifies various parameter settings for the referenced basic models. The classical SES/MB framework only allows static modeling.

To allow dynamic variability modeling and to keep a lean SES tree, the SES/MB framework was extended by several features, such as an interface to the SES and a procedural knowledge representation [7]. In the Research Group Computational Engineering and Automation (RG CEA) at the University of Applied Sciences Wismar a prototype of a SES/MB-based modeling and simulation infrastructure has been developed and implemented within MATLAB/Simulink.

In this paper a short introduction into the extended SES/MB framework is given. It is demonstrated, how the framework can be used to model and simulate a configurable test bench for valves. The main goal is to show that the whole SES can be constructed using elementary design patterns. These in turn can be classified into the categories of the feature relations known from feature models.

\section{Background}

According to [8], the SES is an ontology, a language with syntax and semantics to represent declarative knowledge. It is particularly suitable for describing system configurations for different application domains. 
An SES is represented by a directed tree structure. Objects are represented by nodes which are connected by edges. There are four node types with different properties describing the objects and their relations. Furthermore, there are axioms for defining the SES correctly. Since an SES describes a number of system configurations, the SES tree needs to be pruned to get one particular configuration, which is called Pruned Entity Structure (PES).

The classic SES theory was extended by several researchers over the last decades. Some of these extensions, which are introduced in [7, 9], are used in this paper.

\subsection{Node Types}

Among the four node types, there are two groups, the entity nodes and the descriptive nodes. Entity nodes describe objects of the real or the imaginary world. The root and the leaves of an SES tree are always entity nodes. Relations between the entity nodes are specified by descriptive nodes.

Descriptive nodes are the genus for aspect nodes, specialization nodes and multi-aspect nodes. Aspect nodes describe how entity nodes can be decomposed into partial entities whereas the taxonomy of an entity is described by specialization nodes. Multi-aspect nodes are a special case of an aspect node with all children being of the same kind.

Each node or edge can have attached variables, also called attributes. For entity nodes, the variables represent properties of the respective object whereas the variables at descriptive nodes specify relations between their parent node and children nodes or decisions for the pruning process. With the extended procedural knowledge representation as described in [7, 9], values of attached variables can be assigned dynamically.

\subsection{Axioms}

The semantics of the SES are defined by axioms. The types of the nodes have to follow the axiom alternating mode. Every entity node has to be followed by a descriptive node and vice versa. A strict hierarchy is needed. In every path of the tree, a name of a node may occur only once. If nodes in different paths have the same name, they need to have the same variables and isomorphic partial trees. This is called uniformity. Nodes on the same level of hierarchy, called sibling nodes, have to be valid brothers, meaning that sibling nodes must not have the same name.
The axiom of attached variables implies that a node must not have variables of the same name. The axiom of inheritance implies, that during pruning the parent and the child of a specialization combine their attributes. If parent and child have the same attributes, the parent's attributes are overwritten with the child's attributes and their values.

\subsection{Extended SES/MB Infrastructure}

The SES describing a set of system designs has been associated with the idea of model generation of modular, hierarchical systems from the very beginning (Zeigler, Praehofer, and Kim 2000) which led to the SES/MB approach. Each system design is defined by its system structure and parameter configuration in the SES. The core assets of all system variants are specified as a set of configurable basic models, which are organized in a Model Base (MB).

The classic SES/MB framework defines a set of transformation methods for generating executable simulation models, but automated model generation is not provided. To allow automated generation and execution of models, the SES/MB approach has been extended [7, 9, 10]. These extensions make the SES/MB approach more pragmatic for implementation and to be used in a simulation infrastructure.

Figure 1 depicts the extended SES/MB infrastructure consisting of the SES/MB framework, an Execution Unit, and an Experiment Control. Although the SES/MB approach and its extensions are usually considered in connection with the generation of simulation models, they are generally applicable to modularhierarchical structured software systems.

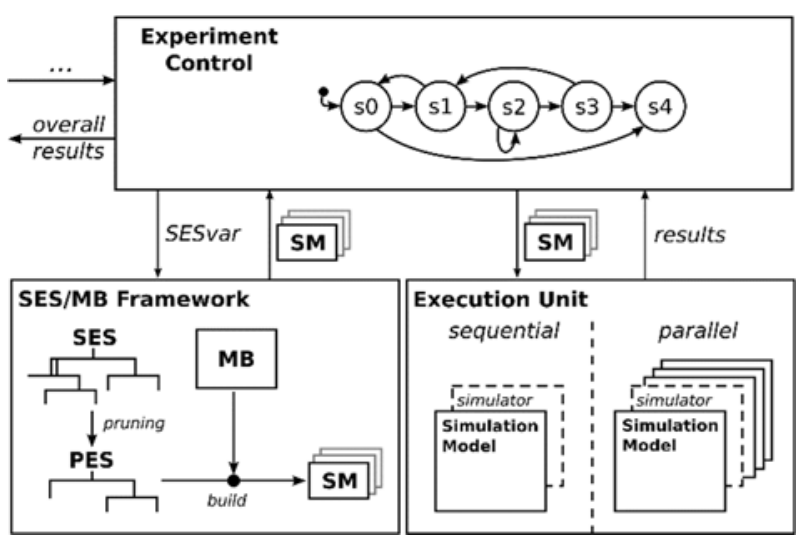

Figure 1: Extended SES/MB-based infrastructure. 


\section{Operations.}

On the SES, a merge operation is defined allowing two or more SES to be combined. This allows the quick reuse of a once defined SES. The essential operation on the SES is the pruning method. To extract one particular system structure and configuration, the SES needs to be trimmed to a PES. During the pruning process, decisions have to be taken at descriptive nodes. Therefore, rules need to be defined at aspect, multi-aspect and specialization nodes. The specialization rule (specrule) associated with a specialization node determines which child entity shall be selected. Aspect rules (aspectrule) associated with aspect or multi-aspect nodes on the same hierarchy level determine which of the siblings is to be chosen.

Furthermore, cross-tree relations can be expressed by selection constraints. Selection constraints can be used to select a certain entity based on decisions taken anywhere else in the SES tree. Next to the pruning method, another transformation method is the build method. With the help of the build method, an executable model can be built from a PES and basic models organized in an MB. The basic models are specific for a certain simulation software. Therefore, the build method needs to match the simulator used.

\section{Execution Unit and Experiment Control.}

For automated and reactive processing of SES models, an execution unit and an overall experiment control unit are added to the framework, as depicted in Figure 1. For automatic generation of different PES, leading to different simulation models, an interface to the SES is needed. This interface can be established using global variables of the SES, called SES Variables (SESvar), which can affect the decisions taken in descriptive nodes during pruning. Thus, a particular system configuration derived from an SES depends on the current settings of the SES variables. The value range of SES variables can be limited by defining semantic conditions, which are checked before pruning to exclude certain system configurations.

By assigning values to the SES variables, the experiment control determines the order and system configurations of executable simulation models (SM) to generate from the SES with the pruning and build operations. Thereby, different variants of the executable simulation models are generated. The experiment control then transmits the SM to the execution unit.
The execution unit links the generated simulation model to the simulator, executes a simulation run and, finally, sends the results back to the experiment control. The results, in turn, can influence the decision of experiment control on how to assign the SES variables next.

\section{Special Attributes.}

Combining basic models from the MB leads to the creation of coupled models. In order to describe the structure of the executable model, some nodes need to define couplings. Couplings are properties of descriptive nodes of the type aspect and multi-aspect and consist of pairs of entity names and port names. They describe causal or acausal relationships. Furthermore, for a multi-aspect node, a special variable, numRep, has to be defined representing the number of children to generate when pruning this node. To specify the basic model from the MB an entity node refers to, the mb-attribute is introduced. This special attribute is permitted just for leaf nodes. Finally, for some cases, it is necessary to define priorities among descriptive nodes on the same level of hierarchy in the priority attribute. All values of attributes can be defined by constants or set via SES variables or SES Functions.

SES Functions. The concept of SES Functions (SESfcn) has been introduced to specify complex variability within node attributes with minimal effort and to keep a lean SES tree. Typical examples include the definition of varying coupling relations, varying port numbers of systems or the definition of variable parameter configurations in attributes. During pruning, SES functions are evaluated, often with SES variables as input parameters. For effective coding of SES functions, the implicit attributes parent and children are introduced for each SES node. They encode the parent and children node names, respectively.

\subsection{Software Tools for the Extended SES/MB Infrastructure}

In the Research Group CEA, a prototype tool for the SES/MB infrastructure was developed, The SES Toolbox for Matlab/Simulink [10]. Currently, SES trees can be defined via a graphical user interface and a concrete variant can be extracted by pruning. The toolbox supports the modeler with plausibility tests during SES construction, graphical representation of the SES, automatic generation of HTML documentation, and other features. 
The pruning process can be started from the graphical user interface and, in addition, is implemented to function automatically. Automatic pruning is necessary when using an SES constructed with the toolbox together with the experiment control. Furthermore, there is a prototype Matlab function implementing a build method for the simulation software Simulink, including Sim Events and Simscape, the MatlabDEVS toolbox [11], and for Modelica models. The SES is linked to the appropriate $\mathrm{MB}$ with the special mb-attribute of the leaf entity nodes.

Another software tool based on Python3/PyQt5 is under development. The aim is to be more independent from a computing environment and to support a greater number of simulators for building executable simulation models.

\section{An Engineering Application Specified with the Extended SES/M B Framework}

For development and testing of the pruning algorithm used in the software tools, design patterns were found. Design patterns are rather small SES fragments with a certain behavior when pruned. They can be used to compose complex SES. The design patterns identified can be classified according to the feature relations in feature models. In the context of feature modeling, four kinds of features are used: (i) mandatory features (logical AND), (ii) alternative features (logical XOR), (iii) optional features and, (iv) OR-features (logical OR). In an SES mandatory and alternative features can be expressed in several ways [12].

In order to be able to describe optional features an extension of the SES is used, the NONE element [8]. A NONE element as a leaf entity node means that, if the NONE branch is selected, the entity is not included at all. OR features can only be expressed by the combination of an aspect node with two or more specialization nodes. Each of the specializations needs to have one NONE element as child.

In this section, an engineering example describing a test bench for valves is given, here called industrial plant (industrialPlant) for generalization. The SES of the entire plant structure and the corresponding process controls is depicted in Figure 2, but without a detailed specification of coupling attributes.
Since different valve types shall be tested, there is a need for a variability in structure for different test procedures. The example shall demonstrate how a complex SES tree can be composed of design patterns, how the SES can be pruned to extract one variant in the form of a PES, and how an executable model can be built from the PES. The feature relations, except for the OR relation, can be found in the SES. Due to complexity, the SES is split up into several SES, depicted in several figures, to increase clarity and to demonstrate modular SES-based modeling.

\subsection{Main SES}

The plant is a composition of the plant structure and the necessary process control strategies. Firstly, the plant structure (plantStructure) shall be explained. The plant consists of the plant parts for gas (gas), liquid (liquid), the electrical power supply (electricalEnergy) and finally, there are controllers to drive the plant. The gas and liquid entities are decomposed further whereas the electrical energy (electricalEnergy) is a merge point to the SES in Figure 6. Merge points are written in bold letters in Figure 2.

As described, merge points allow to combine two SES, which are specified seperately. A merge point is characterized by the same entity node name in both SES, which shall be combined. This implies, that both SES are defined completely and fulfill all axioms.

The mandatory relations described by the aspect node plantStructureDec represent the simplest design pattern for pruning, since the resulting PES is identical to the SES. We call it design pattern \#1. The design patterns are numbered according to the classification found in [12] and pruning is explained for them in the following. One resulting PES representing one test bench configuration is discussed in section 2.8.

Pressure vessels (pressureVessels) as well as compressors, valves, pumps and controllers are entities to subsume a varying number of identical children. They are the parents of a multiple aspect, so during pruning a number of their respective children is created. The entity controllers for example consists of a number of the entity controller. The number is defined in the variable numRep. This is another mandatory feature with regard to feature models. It is referred to as design pattern \#2. Pressure vessel (pressureVessel), compressor, valve, and pump are merge points to SES specified later. 


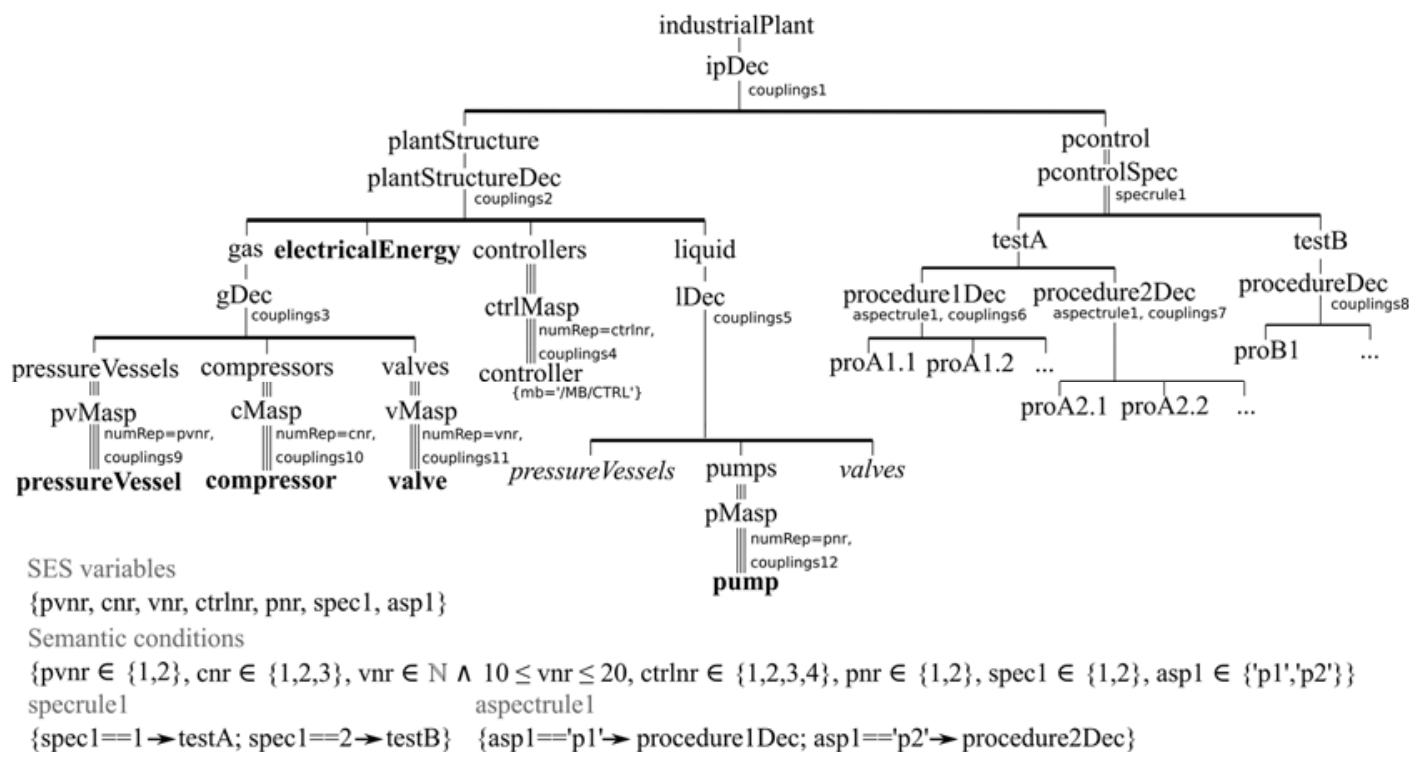

Figure 2: Main SES of the test bench for valves (without detailed coupling definitions).

As described before, one SES axiom urges nodes in different paths with the same name to have the same variables and isomorphic partial trees. Therefore the entities pressureVessels and valves in the branch for the liquid (written italic in Figure 2) do not need to be specified since they are specified for the partial tree defining the gas.

Before listing the sub SES for merge points, the process control part in the main SES shall be explained. In a derived variant of the SES, either testA or test $B$ can be part of the PES to specify a particular system configuration. This is an alternative selection, called design pattern \#4. Therefore, the entities test $A$ and test $B$ are specializations of the process control that is represented by the entity pcontrol. For decision, which test to execute, a specialization rule (specrule) has to be defined at the specialization node pcontrolSpec. During pruning the specrule is interpreted and the selected testA or testB entity is united with the parent entity pcontrol.

If testA is selected, there are two possible main procedures which can be taken alternatively. The decision is taken by evaluation of aspectrule1 at procedurelDec and procedure $2 \mathrm{Dec}$. This design pattern for an alternative selection is referred to as pattern $\mathbf{\# 5}$. The decision taken leads to a number of elementary procedures (e.g. proA1.1...proA1.n) specific for the selected superordinate main procedure. For testB there is only one main procedure leading to a number of elementary procedures as explained in design pattern \#1.
The SES in Figure 2 demonstrates the use of SES variables (SESvars) for dynamical generation of different system configurations. SESvars can contain any type of value. At nodes needing a decision during pruning, the corresponding attribute or rule can be defined based on an SESvar, so each value can be assigned dynamically. The value range of every SESvar should be restricted using semantic conditions. A semantic condition needs to evaluate to a logical value and can contain more than one logical expression. SESvars are assigned to the numRep attributes at the multi-aspect nodes. There are the SESvars spec and asp used in the specrule and the aspectrule respectively. If the SESvar spec equals 1 , test $A$ is taken, if spec equals 2 , test $B$ is selected during pruning. The SESvar asp can take one of two possible strings as value. The aspectrule defines that, if asp equals the string $\mathrm{p} 1$, procedurelDec is selected, and if asp equals the string $\mathrm{p} 2$, procedure $2 \mathrm{Dec}$ is taken.

The aspect and multi-aspect nodes need the special attribute couplings as seen in Figure 2. They describe the relations of entity nodes. An example for a coupling definition is given in section 2.9. In this context the usage of SES functions is illustrated as well.

\subsection{Sub SES: Pressure Vessel}

In Figure 3 the pressure vessel (pressureVessel) is specified. Every pressure vessel has the two attributes volume and maxPressure. Creating the PES depending on the specrule defined, either steel or aluminum is taken as material, which is united with the pressureVessel entity. 


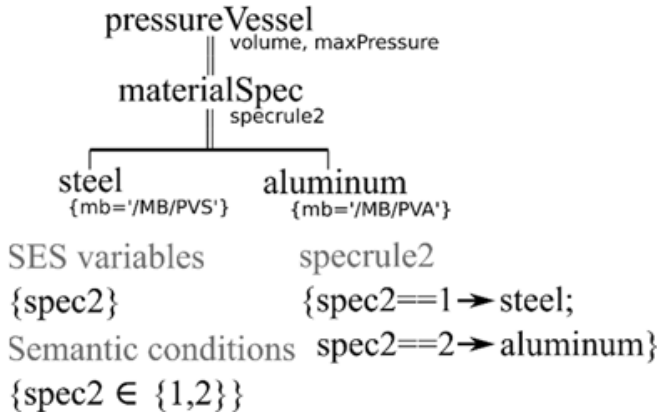

Figure 3: SES of a pressure vessel.

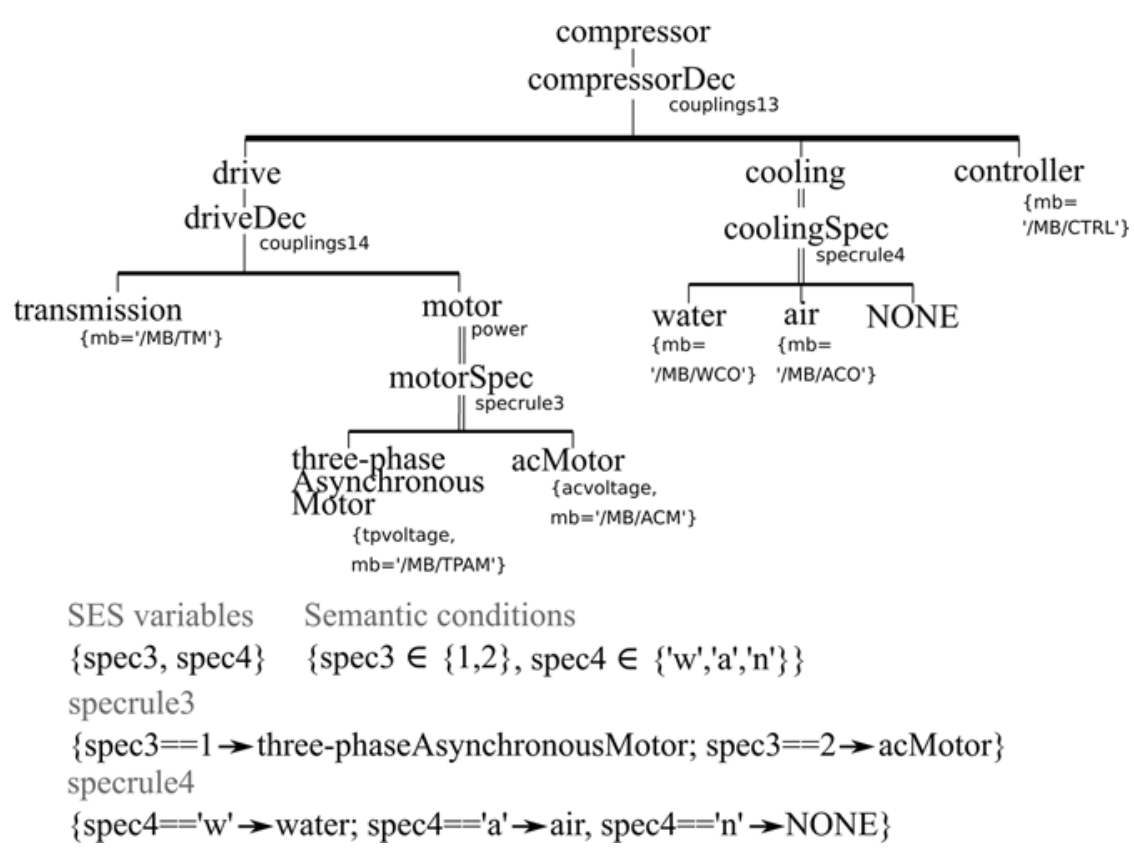

Figure 4: SES of a compressor.

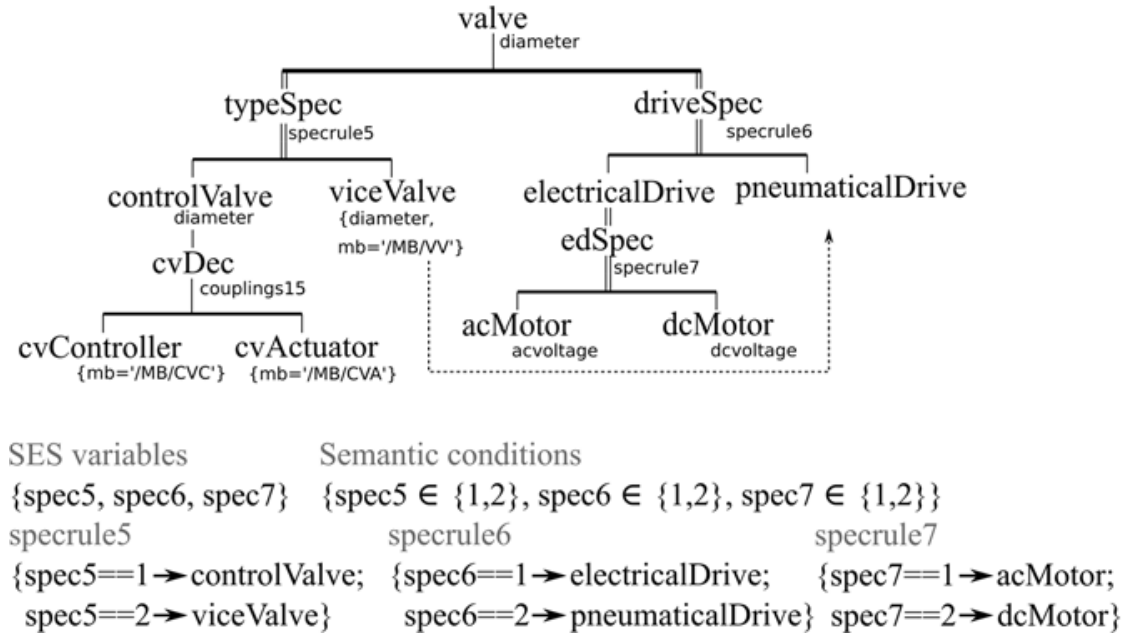

The mb-attribute of the leaf entities refers to a basic model in the MB, which is depicted in Figure 8. During pruning attributes are inherited to the parent, which is renamed and has three attributes afterwards. This is another example for the design pattern \#4 with the addition of attribute inheritance. As in the main SES, the decision at the specialization is taken based on the specrule and the current values of SES variables.

\subsection{Sub SES: Compressor}

The details of the compressor can be seen in Figure 4. A compressor is composed of the drive, the cooling and a controller. The drive is composed of the motor and a transmission. This is an SES construction as described in design pattern \#1. The motor can be specified as a three-phase asynchronous motor or an ac motor, which refer to a model in the MB with their mb-attribute. During pruning, this part is resolved as described for the pressure vessel in design pattern \#4.

The cooling is an optional element. Since it is a specialization with a NONE element, the pruning process resolves the cooling either to water cooling, air cooling or no cooling at all. The optional tree section is referred to as design pattern $\mathbf{\# 8 .}$

\subsection{Sub SES: Valve}

Valves are needed for the plant parts for gases as well as for liquids and are only defined in the partial SES defining the gas as described before. A valve is of a type and needs to have a drive. The specialization siblings type Spec and driveSpec in Figure 5 can be classified as mandatory feature and are referred to as design pattern \#3.

Figure 5: SES of a valve. 
The entity electricalDrive is specialized into acMotor and dcMotor according to design pattern \#4. During the pruning process all specializations are resolved, attributes are inherited and the entity valve is renamed with the selected type, drive and, if the electricalDrive is selected, the motor type. Two specialization nodes in one path as seen in this example is a combined design pattern referred to as design pattern \#10 and is showing attribute inheritance among several layers. In section 2.8 the inheritance of names and attributes is explained in detail. The controlvalve is composed of the cvController and the cvActuator. Seen from the top entity valve, this is another combined design pattern consisting of a specialization with a succeeding aspect. We call it design pattern \#11. It shows that during pruning the specialization typeSpec is resolved according to design pattern \#4 and the aspect $c v D e c$ is not changed like in design pattern \#1. The dotted line in Figure 5 is a selection constraint for cross-tree relations, which forces the use of a pneumaticalDrive, if a viceValve is selected. It may be noticed, that in Figure 5 not all leaf nodes have an mb-attribute. Either the leaf nodes in the branch of the typeSpec entity or the leaf nodes in the branch of the driveSpec are allowed to have mb-attributes. The other branch can only configure the basic model. In this example the mb-attribute is set in the typeSpec branch, while the configuration is set in the driveSpec branch. During pruning identical attributes are overwritten in parent nodes, so would be the mb-attribute if defined for both branches.

\subsection{Sub SES: Electrical Energy}

The SES describing the electrical energy is shown in Figure 6. The electrical energy (electricalEnergy) can be generated with multiple generators specified in the variable numRep or the electrical grid ( ridDec) is used.

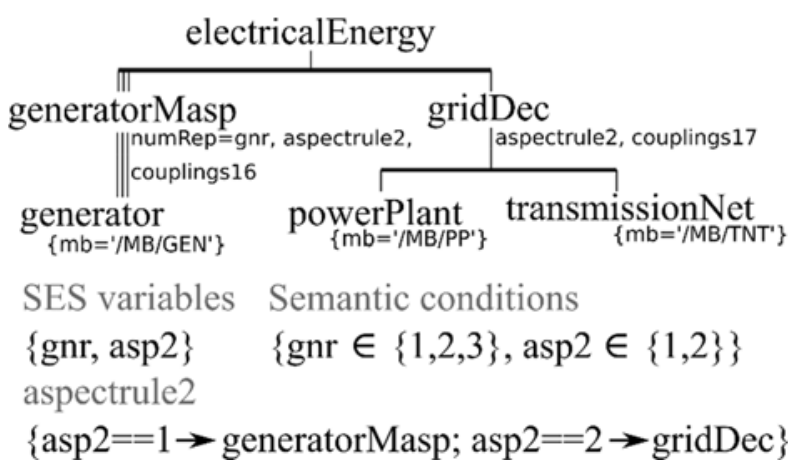

Figure 6: SES of the electrical energy
The grid is decomposed into a power plant (powerPlant) and the transmission net (transmissionNet). The decision, whether the generator or the grid is selected, is taken in aspectrule2. This alternative structure created of siblings of aspect and multi-aspect nodes is called design pattern \#7.

\subsection{Sub SES: Pump}

The pump can be built with the SES depicted in Figure 7. It is decomposed into the motor, the wheel and a controller just like design pattern \#1. The motor does not need to be specified since it is already defined in the SES of the compressor (see Figure 4).

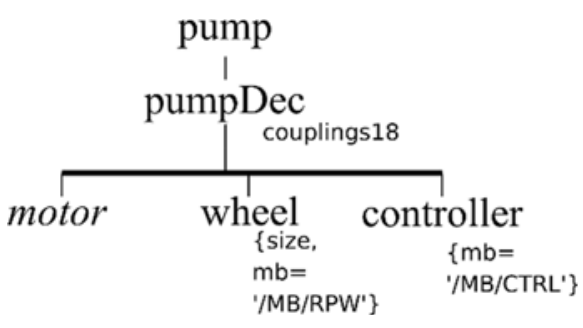

Figure 7: SES of a pump.

\subsection{Model Base}

For model generation a library containing the configurable basic models specified in the mb-attribute of leaf nodes is needed. A possible model base (MB) for this example is given in Figure 8. The MB contains the dynamic models for simulation of the respective devices. Every model needs input and output ports for building coupling relations. A basic model in the MB can be composed of several models as depicted for the $T N T$ model.

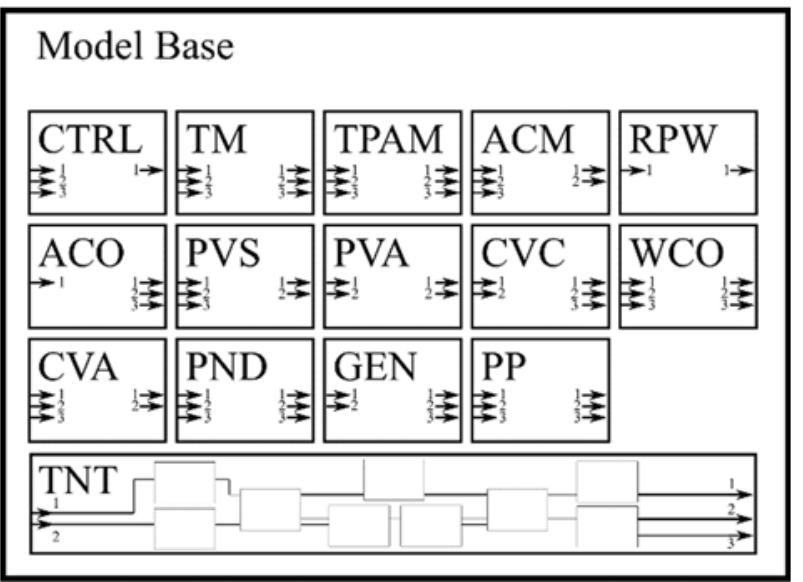

Figure 8: M odel Base for this example. 


\subsection{Deriving a PES}

Since an SES specifies a number of different system configurations, the SES has to be transformed into a PES by pruning in order to get one particular system configuration. Figure 9 shows a valid SESvars setting and the derived PES, which represents one variant of the configurable test bench for valves. Before pruning, values are assigned to the SESvars and checked for validity using the semantic conditions. The process of resolving all necessary decisions is described in the following.

First, it shall be described how to derive a particular variant from the main SES in Figure 2. Since the resulting PES for pruning pattern \#1 is identical to the SES, the composition of the plant (industrialPlant) as well as the composition of the plant structure (plantStructure), the gas (gas) and the liquid (liquid) are part of the PES. The multi-aspect nodes pvMasp, cMasp, vMasp, ctrlMasp, and pMasp are resolved following design pattern \#2 by converting them into aspect nodes with the name extension Dec and a certain number of children is created as defined in numRep. The number of each child is appended to the nodename of the child.

For the electrical energy (electricalEnergy) the selection for generatorMasp is taken in the aspectrule2 (design pattern \#7) and the respective children (generator) are created as in design pattern $\mathbf{\# 2}$.
The pressure vessel is pruned according to design pattern \#4. As material of the pressure vessel (pressureVessel) aluminum is selected by evaluating specrule2. The name of the selected entity (aluminum) is united with the parent of the specialization (pressureVessel) to aluminum_pressureVessel, attributes of the child are inherited to the renamed parent and the specialization node materialSpec is deleted.

The compressor drive (drive) can be pruned using design pattern \#1 and design pattern \#4 as before. The cooling is a specialization evaluating to NONE according to design pattern \#8. The NONE node is depicted in Figure 9 for understanding, but has no relevance for the succeeding model generation.

For generating the PES of a valve based on the SES in Figure 5, both specialization siblings have to be resolved, the resulting names are connected and the attributes are inherited (design pattern \#3). It depends on the pruning algorithm used, which of both specializations is pruned first. There is no explicit rule. In this example the pruning is started with the specialization typeSpec. The type controlValve is selected and an intermediate parent entity controlvalve_valvel can be assumed. The value of the attribute diameter in the parent node is replaced with the value of the same attribute in the child according to design pattern \#4.

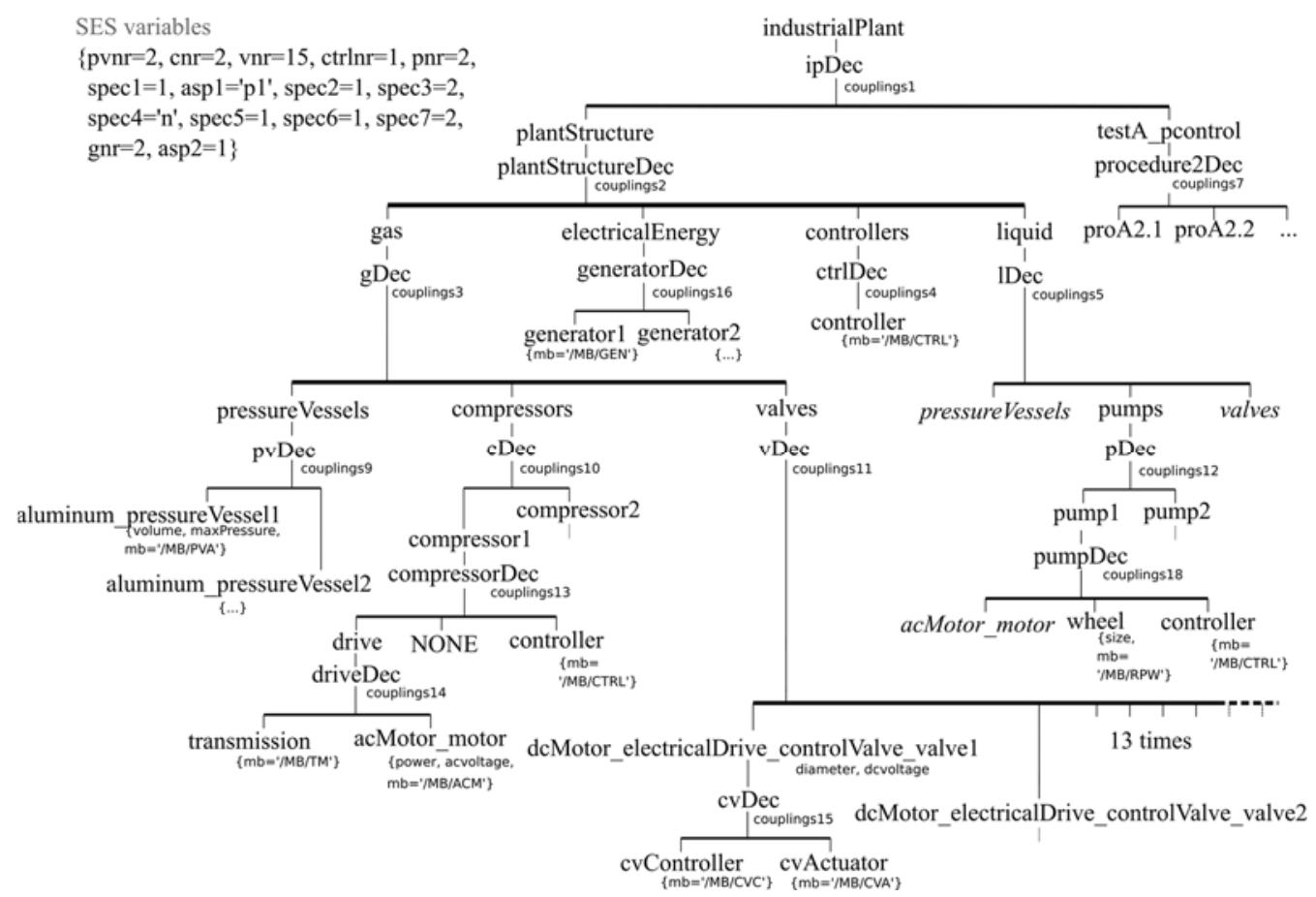

Figure 9: PES describing one particular system configuration derived based on the current SESvars settings. 
The tree beginning with $c v D e c$ is a composition, which is not changed during pruning (design pattern \#1).

Since electricalDrive is selected in driveSpec, both of the specializations in one path (design pattern \#10) driveSpec and edSpec need to be resolved like in design pattern \#4. The entity node dcMotor is selected, the attribute dcvoltage is moved to the top entity, which is renamed to dcMotor_electricalDrive_controlValve_valvel according to the selections.

The entities pressureVessels and valves in the branch for the liquid (written italic in Figure 9) are specified for the partial tree defining the gas and do not need to be specified again. That implies, that in this design the pressure vessels and the valves have to be of the same type and number and need to have the same properties for both plant parts.

A pump as depicted in Figure 7 is a composition of a motor, a wheel and a controller and is pruned according to design pattern \#1. However, since the motor of the pump is the same entity as the motor of the compressor caused by the same name in the SES, in the PES both nodes need to have the same name as well.
The process control (pcontrol) is derived by evaluation of specrule1 like in design pattern \#4 and the aspectrule1 for aspect siblings (design pattern \#5). The entity node testA is selected and united with pcontrol. Finally the aspect node procedure2Dec is selected, which is the child of the renamed testA_pcontrol.

Summarized, in this configuration the industrial plant has four pressure vessels, two compressors, thirty valves, two pumps, two generators and one controller for the plant. Each device of these device groups has the same structure and is of the same type.

However, in the case of the motors of the compressors and pumps it makes sense to relax the axiom of uniformity, so that different values can be assigned to the power attribute. The relevance to relax the uniformity attribute is discussed in [7] comprehensively.

Since the PES is a decision-free tree structure, a particular executable model can be generated based on its information and using basic models from the MB.

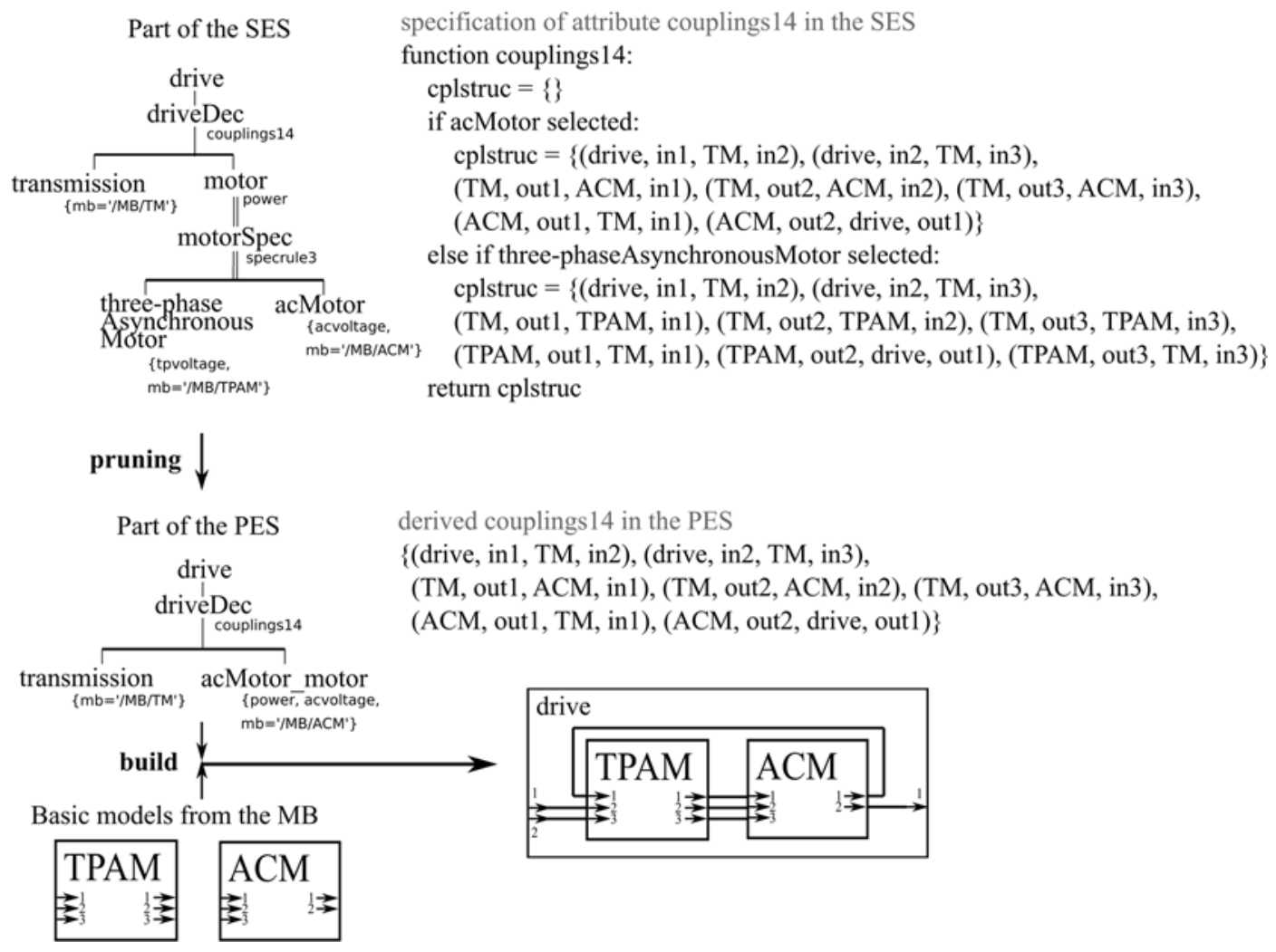

Figure 10: Specification of dynamic couplings in an SES, derived static couplings in the PES, and resulting executable model. 


\subsection{Building the Executable Model}

For the generation of the executable model, the coupling attributes are especially important, as discussed in section 1.3. Examples on how couplings are specified and resolved are depicted in Figure 10 according to the SES part of a drive of a compressor in Figure 4.

In the SES couplings often have to be defined dynamically, since the SES describes several configuration variants. During pruning, one particular configuration with static couplings is derived, so that an executable model can be built. Dynamic specification can be done using SESfcns as illustrated in pseudocode in Figure 10. In the example, the attribute couplings14 in the SES has to specify the coupling relations for both types of basic motor models with a different number of ports. In the PES the concrete static couplings for the selected basic models are derived, in this case for the basic model ACM.

Particularly, when defining the couplings for a multi-aspect node or for nodes referring to basic models, whose port numbers vary depending on the selected configuration, defining the couplings via SESfens is best practice.

\section{Conclusion}

This paper discusses how to use the extended SES/MB framework in order to describe and build a set of simulation models of a complex configurable engineering application by the example of a test bench for valves. It is shown, that previously found design patterns are appropriate to specify a complex engineering problem. Such engineering problems can be modeled using the proposed software tools for the extended SES/MB infrastructure, executable models for different simulators can be built and finally simulated.

\section{Acknowledgements}

The authors acknowledge the grant from the German Science Foundation, DFG (PA 631/2). Moreover, the authors would like to thank Peter Junglas, who contributed valuable work to the development of a generic model builder for the SES toolbox; Daniel Pascheka, who implemented a first version of the graphical editor within MATLAB; Birger Freymann, who redesigned the editor and implemented a model builder for the MatlabDEVS toolbox and our former colleague, Tobias
Schwatinski, who provided preliminary work. Last, but not least, we would like to thank Bernie Zeigler for motivating and supporting our work.

\section{References}

[1] Alt O. Variantenmanagement. In: Modellbasierte Systementwicklung mit SysML. Munich: Hanser, 2012. p 6 total pages of chapter.

[2] Kang KC, Lee H. Variability Modeling. In: Capilla, R., Bosch, J., Kang, K. C., editors. Systems and Software Variability Management. Berlin: Springer; 2013. p 7 total pages of chapter.

[3] Kang KC, Cohen SG, Hess JA, Nowak WE, Peterson AS. Feature-Oriented Domain Analysis (FODA) Feasibility Study. Technical Report CMU/SEI-90-TR-21, ESD-90-TR-222, SE Inst. Carnegie Mellon Univ. Pittsburgh/PA, USA. 1990.161 p.

[4] Kang KC, Lee H. Binding Time and Evolution. In: Capilla R, Bosch J, Kang KC. editors. Systems and Software Variability Management. Berlin: Springer; 2013. p 7 total pages of chapter.

[5] Zeigler BP. Multifaceted Modelling and Discrete Event Simulation. 2nd ed. London: Academic Pr.; 1984. 372 p.

[6] Zeigler BP, Kim TG, Praehofer H. Theory of Modeling and Simulation. 2nd ed. Cambridge: Academic Pr.; 2000. 510 p.

[7] Pawletta T, Schmidt A, Zeigler BP, Durak U. Extended Variability Modeling Using System Entity Structure Ontology within MATLAB/Simulink. In Proc. of Spring Simulation Multi-Conference 2016. Spring Simulation Multi-Conference; 2016 Apr; Pasadena/CA, USA. SCS Int. p 62-69.

[8] Zeigler A, Hammonds PE. Modeling and SimulationBased Data Engineering. 1st ed. Academic Pr.; 2007. $448 \mathrm{p}$.

[9] Schmidt A, Durak U, Pawletta T. Model-Based Testing Methodology Using System Entity Structures for MATLAB/Simulink Models. SIMULATION: Transactions of The Society for Modeling and Simulation International. 2016; 92(8): 729-746.

[10] RG CEA. The SES Toolbox for MATLAB/SIMULINK Website. http://www.cea-wismar.de/tbx/SES_Tbx/.

[11] RG CEA. The PDEVS Toolbox for MATLAB Website. http://www.cea-wismar.de/tbx/DEVS_Tbx/ MatlabDEVS_Tbx.html.

[12] Deatcu C, Folkerts H, Pawletta T, Durak U. Design Patterns for Variability Modeling Using SES Ontology. In Proc. of Spring Simulation Multi-Conference 2018. Spring Simulation Multi-Conference; 2018 Apr; Baltimore/MD, USA. SCS Int. 10 p. (paper submitted). 


\title{
Microscopic Pendulum Modelling: Absurd Idea or Innovative View on Old Problems?
}

\author{
Martin Bicher ${ }^{1 *}$, Niki Popper ${ }^{2}$ \\ ${ }^{1}$ Institute for Analysis and Scientific Computing, TU Wien, Wiedner Hauptstraße 8-10, \\ 1040 Vienna, Austria; ${ }^{*}$ martin.bicher@tuwien.ac.at \\ ${ }^{2}$ dwh Simulation Services, dwh GmbH, Neustiftgasse 57-59, 1070 Vienna, Austria
}

SNE 27(4), 2017, 177-182, DOI: 10.11128/sne.27.tn.10392 Received: October 20, 2017, Revised: December 3, 2017, Accepted: December 10, 2017

SNE - Simulation Notes Europe, ARGESIM Publisher Vienna, ISSN Print 2305-9974, Online 2306-0271, www.sne-journal.org

Abstract. Especially in the last years the idea of finding a simpler meta-model for a developed simulation model has become more and more popular as not only the research questions, but also the resulting models have become more and more complex. The meta-model hereby helps understanding the behaviour of the original model and supports its validation and verification process. Moreover, it also gives a second perspective on the modelled system. Mean-field theory is a very formal link between microscopic and macroscopic models and can be used to find meta-models for either of the two types. Usually so-called mean-field analysis is used to find a summarising simpler macroscopic model for a given complex microscopic approach, but we will emphasise the inverse process in this work: Applying inverse mean-field analysis on an ordinary differential equation model we systematically derive a microscopic representation of the model. On purpose we chose a very unusual model to apply the method to: the un-damped linearised pendulum equation. Hereby we want to emphasise the method's flexibility and generality, investigate possible benefits of the gained microscopic meta-model for this strange field of application, and discuss about interpretation of the elements of the resulting agent-based pendulum model.

\section{Introduction}

According to German philosopher Herbert Stachowiak [1] any model, in general, is defined as a (usually) simplified image of a real or artificial object that has been developed for a specific purpose and time of usage.
This definition clearly permits the idea of developing a model $M_{2}$ for a different model $M_{1}$, a so-called meta-model, developed for a specific purpose related to $M_{1}$. For simulation models this strategy becomes more and more popular nowadays due to steadily increasing complexity of research questions and models. Hereby the more simplified meta-model $M_{2}$ can be used to either get insights into $M_{1}$ to simplify its verification and validation process (Compare with Bernhard Zeigler's talk "Why Should We Develop Simulation Models in Pairs" at WSC 2017 [2]) or to get new ideas about the modelled system.

Mean-Field Approximation (MFA) is one concept for finding such a meta-model for a given microscopic model. Hereby chosen aggregated numbers of the microscopic model $\left(M_{1}\right)$ are approximated by solutions of specific ordinary or partial differential equations - the so-called mean-field model $\left(M_{2}\right)$. For detailed information the reader is referred to [3]. One key advantage of this method is that the meta-model is (in principle) found by a formal recipe that guarantees the "correctness" of the meta-model with respect to (asymptotic) equivalence of the model results. The key to this equivalence lies within a formal theorem, generally referred to as Mean-Field Theorem (MFT), which states how the elements of the microscopic model need to be used to find in macroscopic equation-based meta-model, the so-called mean-field model. The formality of this procedure makes the strategy extremely generic and completely independent of the investigated model's field of application. It can e.g. be applied to find mean-field models for agent-based models in economics [4] or sociology [5] as well as for cellular automata models in epidemiology [6, 7] or population dynamics [8].

Another example that emphasises the generality of the method is gained interpreting mean-field approximation as an invertible mapping from a microscopic to a mean-field model. Inverse MFA is presented in [3], Chapter 5.2.3, on the example of the Levins Model 
[9], a macroscopic model for the growth of a population. Using this inverse strategy, a microscopic representation of this non-linear ordinary differential (ODE) model is derived systematically. Hereby the role of the meta-model $M_{2}$ is switched from the macroscopic to the microscopic one. Although a meta-model in form of a microscopic approach, e.g. in form of an agent-based model, can hardly be considered particularly easier than the original macroscopic one, it is definitely more flexible and avoids specific problems with the numerics of e.g. ODE solvers. Moreover, it gives a very interesting view on the original system as the microscopic elements can be interpreted as single agents.

In this work we want to apply inverse MFA on a much more abstract example: the linearised, undamped pendulum model given by

$$
\ddot{\varphi}(t)=-k \varphi(t), \varphi(0)=\varphi_{0}, \dot{\varphi}(0)=0,
$$

with $k>0$. Although, in the contrary to the Levins model, there is no feasible justification for modelling a pendulum in a microscopic way we will apply this formal method to

a) emphasise its flexibility and generality by showing that it actually works,

b) investigate possible benefits of the gained microscopic meta-model for this particular application, and

c) attempt to interpret the microscopic elements of the resulting meta-model.

\section{Inverse Mean-Field Approximation}

In order to perform the mentioned inverse MFA we will stress the strategy proposed in [3] Chapter 4.6.4. Key idea of this strategy is finding suitable transformations, so that the equation of the ODE model has the form of the system

$$
\begin{gathered}
\frac{d \vec{Y}_{1}}{d t}=\sum_{j=1}^{d} \vec{Y}_{j} \omega_{j, 1}(t, \vec{Y})-\vec{Y}_{1} \omega_{1, j}(t, \vec{Y}) \\
\vdots \\
\frac{d \vec{Y}_{d}}{d t}=\sum_{j=1}^{d} \vec{Y}_{j} \omega_{j, d}(t, \vec{Y})-\vec{Y}_{d} \omega_{d, j}(t, \vec{Y})
\end{gathered}
$$

of ordinary differential-equations (ODEs) with parameter functions $\omega_{i, j} \geq 0, i, j \in\{1, \ldots, d\}$ and sufficiently large positive solutions $\vec{Y}(t)$. In case such a representation has been found, the parameter functions $\omega$ can be used as transition rates of the microscopic elements of a microscopic model. Hereby $\omega_{i, j}$ denotes the rate (transition-rate) that an individual with state $i$ switches to state $j$. Let $a_{k}(t) \in\{1, \ldots, d\}$ denote the time dependent state of individual $k$ in the newly defined microscopic model with $N$ individuals in total, then (a version of) the Mean-Field Theorem (Corollary 5.1 in [3]) states that the aggregated numbers

$$
\vec{X}_{i}(t):=\sum_{k=1}^{N} \mathbb{1}_{i}\left(a_{k}(t)\right), \quad i \in\{1, \ldots, d\}
$$

fulfil $\vec{X}_{i}(t)=\vec{Y}_{i}(t)+\mathscr{O}(\sqrt{N})$, wherein $\vec{Y}$ is the solution of above system of ODEs. Note, that $\vec{X}$ is precisely the total number of microscopic elements that share the same state at time $t$. In case $\omega_{i, j}$ depends on $\vec{Y}$ in the ODE system, the individual transition rates depend on the aggregated number vector $\vec{X}$ accordingly. The latter can be interpreted as interaction of the microscopic elements on the aggregated level.

In [3] Chapter 4.6.4 a step-by-step instruction gives hints how mentioned transformation can be found. We will mainly follow these proposed steps:

a) First of all, the pendulum equation, a second order ODE, needs to be written as a system of first order ODEs via

$$
\varphi(t)^{\prime \prime}=-k \varphi(t) \Rightarrow\left(\begin{array}{c}
\varphi \\
\psi
\end{array}\right)^{\prime}(t)=\left(\begin{array}{c}
\psi(t) \\
-k \varphi(t)
\end{array}\right)
$$

b) Moreover, the variable-functions appearing in (2) need to be positive which is violated in (4) as angle and angular velocity resulting from the pendulum equation might also have negative values. Therefore, we need to translate the equation via

$$
\left(\begin{array}{c}
\tilde{\varphi} \\
\tilde{\psi}
\end{array}\right):=\left(\begin{array}{c}
\varphi \\
\psi
\end{array}\right)+T
$$

so that $\tilde{\varphi}, \tilde{\psi}$ is always positive. For physically feasible systems this translation is always possible as model trajectories need to be stable. In case of the pendulum it is easy to reason with physical arguments (e.g. Newton's axioms) that $\varphi(t) \in\left[-\left|\varphi_{0}\right|,\left|\varphi_{0}\right|\right]$ and $\dot{\varphi}(t) \in\left[-\sqrt{k}\left|\varphi_{0}\right|, \sqrt{k}\left|\varphi_{0}\right|\right]$ and therefore any

$$
T>\max \left\{\sqrt{k}\left|\varphi_{0}\right|,\left|\varphi_{0}\right|\right\}
$$


is sufficiently large to guarantee that

$$
(4) \Longrightarrow \quad\left(\begin{array}{c}
\tilde{\varphi} \\
\tilde{\psi}
\end{array}\right)^{\prime}(t)=\left(\begin{array}{c}
\tilde{\psi}(t)-T \\
-k \tilde{\varphi}(t)+k T
\end{array}\right),
$$

has positive solutions $\tilde{\varphi}$ and $\tilde{\psi}$.

c) As the variable-functions will end up approximating the aggregated numbers of a microscopic model, we need to rescale the equation to get them sufficiently large. We define

$$
\left(\begin{array}{c}
\bar{\varphi} \\
\bar{\psi}
\end{array}\right):=S\left(\begin{array}{c}
\tilde{\varphi} \\
\tilde{\psi}
\end{array}\right),
$$

leading to

$$
(6) \Longrightarrow\left(\frac{\bar{\varphi}}{\bar{\psi}}\right)^{\prime}(t)=\left(\begin{array}{c}
\bar{\psi}-S T \\
-k \bar{\varphi}+k S T
\end{array}\right) .
$$

d) As (2) conserves the sum

$$
\sum_{i=1}^{d} Y_{i}^{\prime}(t)=0
$$

we introduce a third state $\bar{\xi}$ and write

$$
\text { (7) } \Rightarrow\left(\begin{array}{c}
\bar{\varphi} \\
\bar{\psi} \\
\xi
\end{array}\right)^{\prime}=\left(\begin{array}{c}
\bar{\psi}-S T \\
-k \bar{\varphi}+k S T \\
k \bar{\varphi}-k S T-\bar{\psi}+S T
\end{array}\right) \text {. }
$$

Hereby we need to care for a feasible initial condition. As the sum of the right-hand-sides is zero, the sum of the variable functions is constant $\bar{\varphi}+\bar{\psi}+\bar{\xi}=C$. As $0<\bar{\varphi}<2 S T$ and $0<\bar{\psi}<2 S T$ positivity for $\bar{\xi}$ is ensured if (e.g.) $\bar{\xi}_{0}:=4 S T$.

e) As the transition rates $\omega$ need to be positive functions, we need to rewrite the above modified pendulum equation one last time to finally match the form of (2):

$$
\begin{gathered}
(8) \Longrightarrow \\
\left(\begin{array}{c}
\bar{\varphi} \\
\frac{\bar{\psi}}{\xi}
\end{array}\right)^{\prime}=\left(\begin{array}{c}
\frac{\max (\bar{\psi}-S T, 0)}{\bar{\xi}} \bar{\xi} \\
\frac{\max (-k \bar{\varphi}+k S T, 0)}{\bar{\xi}} \bar{\xi} \\
\frac{\max (-\bar{\psi}+S T, 0)}{\bar{\varphi}} \bar{\varphi}+\frac{\max (k \bar{\varphi}-k S T, 0)}{\bar{\psi}} \bar{\psi}
\end{array}\right)
\end{gathered}
$$

$$
-\left(\begin{array}{c}
\frac{\max (-\bar{\psi}+S T, 0)}{\bar{\varphi}} \bar{\varphi} \\
\frac{\max (k \bar{\varphi}-k S T, 0)}{\bar{\psi}} \bar{\psi} \\
\frac{\max (\bar{\psi}-S T, 0)+\max (-k \bar{\varphi}+k S T, 0)}{\bar{\xi}} \bar{\xi}
\end{array}\right) .
$$

The last transformation (9) finally makes it possible to match (2). We identify and define (for $\bar{\varphi} \rightarrow \vec{X}_{1}, \bar{\psi} \rightarrow$ $\left.\vec{X}_{2}, \bar{\xi} \rightarrow \vec{X}_{3}\right)$

$$
\left(\begin{array}{l}
\omega_{1,3} \\
\omega_{2,3} \\
\omega_{3,1} \\
\omega_{3,2}
\end{array}\right)(t, \vec{X}):=\left(\begin{array}{c}
\frac{\max \left(-\vec{X}_{2}+S T, 0\right)}{\bar{X}_{1}} \\
\frac{k \max \left(\bar{X}_{1}-S T, 0\right)}{\bar{X}_{2}} \\
\frac{\max \left(\bar{X}_{2}-S T, 0\right)}{\bar{X}_{3}} \\
\frac{k \max \left(-\bar{X}_{1}+S T, 0\right)}{\bar{X}_{3}}
\end{array}\right) .
$$

Using these transition rates in a microscopic model, the aggregated numbers of this model with asymptotically equal the solutions of (9). As

$$
\overline{1}\left(\begin{array}{l}
\bar{\varphi} \\
\bar{\psi} \\
\xi
\end{array}\right)-T=\left(\begin{array}{l}
\varphi \\
\psi \\
\xi
\end{array}\right)
$$

an affine transformation (scale $S$ and transition $T$ ) of the aggregated numbers of the first state of the microscopic model approximates the solution of the pendulum equation, the angle of the pendulum. The aggregated number of the second state corresponds to the angular velocity and will also be regarded in our analysis.

Following [3] we introduce a time-step size $d t$ to set up a time-discrete microscopic model. Therefore, for all microscopic elements $a_{k}$ of the new model, we deduce the transition probabilities

$$
P\left(a_{k}(t+d t)=i \mid a_{k}(t)=j, \vec{X}\right):=d t \cdot \omega_{j, i}(t, \vec{X}) .
$$

This can be interpreted as a first order Euler (or EulerMaruyama see [10]) approximation.

\section{Agent-Based Pendulum Model}

Given by the results of the last section, the basis for a microscopic meta-model was developed for the undamped pendulum model. To simplify speech, we consider the established model as an agent-based model $(\mathrm{ABM})$ :

Model 1 (Agent-Based Pendulum Model). With $\varphi_{0}$ and $\dot{\varphi}_{0}$ denoting the initial conditions of the pendulum equation and $k$ the frequency parameter, we define the fol- 
lowing microscopic model via its initialisation phase and its time dynamics.

\section{Initialisation:}

- Define a translation parameter $T>$ $\max \left\{\sqrt{k}\left|\varphi_{0}\right|,\left|\varphi_{0}\right|\right\}$ and a sufficiently large scale parameter $S \in \mathbb{R}^{+}$(usually $S \gg 100$ makes sense).

- Define a time-step length dt small enough to guarantee that the probabilities used in the dynamics below are feasible (i.e. smaller than 1).

- Moreover, calculate

$$
\begin{aligned}
& X_{1}:=\left\lfloor\overline{\varphi_{0}}\right\rfloor=\left\lfloor S\left(\varphi_{0}+T\right)\right\rfloor \\
& X_{2}:=\left\lfloor\overline{\psi_{0}}\right\rfloor=\lfloor S T\rfloor, \text { and } \\
& X_{3}:=\left\lfloor\overline{\xi_{0}}\right\rfloor=\lfloor 4 S T\rfloor .
\end{aligned}
$$

- Setup a model with $N:=X_{1}+X_{2}+X_{3}$ agents of which each is assigned one of three states, henceforth denoted as angle (state 1), velocity (state 2) and idle (state 3). Assign $X_{1}$ agents in state 1, $X_{2}$ and $X_{3}$ agents in state 2 and 3 accordingly. The total number of agents in any of the three states is tracked throughout the model using these three variables $X_{1}, X_{2}$ and $X_{3}$.

\section{Dynamics:}

- The model is updated in equidistant time-steps of length $d t$.

- Each time-step, each agent is addressed once. According to its prior state, specific probabilities decide if the agent's state remains or changes.

- In case an agent is in the angle-state and $\vec{X}_{2} \leq S T$, it may switch to the idle-state with probability

$$
d t \cdot \omega_{1,3}=d t \frac{S T-X_{2}}{X_{1}} .
$$

Otherwise, it remains in its state. In case of a switch, $\vec{X}_{3}++$ and $\vec{X}_{1-}$.

- In case an agent is in the velocity-state and $\vec{X}_{1} \geq$ $S T$, it may switch to the idle-state with probability

$$
d t \cdot \omega_{2,3}=d t \frac{X_{1}-S T}{X_{2}} .
$$

Otherwise, it remains in its state. In case of a switch, $\vec{X}_{3}++$ and $\vec{X}_{2}-$.
- In case an agent is in the idle-state and $\vec{X}_{2}>S T$, it may switch to the angle-state with probability

$$
d t \cdot \omega_{3,1}=d t \frac{X_{2}-S T}{X_{3}}
$$

or, in case $\vec{X}_{1}<S T$, to the velocity-state with probability

$$
d t \cdot \omega_{3,2}=d t \frac{S T-X_{1}}{X_{3}}
$$

Otherwise, it remains in its state. In case of a switch, change the aggregated numbers $X_{1}, X_{2}$ and $X_{3}$ accordingly.

The flow-chart in Figure 1 shows how the agents may change their states: They may switch from states "angle" and "velocity" to state "idle" and vice versa. They never switch between "angle" and "velocity" directly.

Moreover, the concept that the transition probabilities of this model depend on the aggregated numbers can be translated as a kind of global-level-interaction between the agents. It is comparable with an economical model of the stock-market: the stock-price is a globally known variable that influences the buying behaviour of all actors. Yet it changes in response to transactions, i.e. to individual behaviour. In the Levins Model presented in [3] in Chapter 5.2.3 the author referred the resulting global-level-interactions in this model to local-level-interactions - i.e. typical agentagent contacts - via stochastic equivalences comparable to picking balls from urns. In this case, we did not attempt to find such a representation as it would be too complicated (but surely not impossible). Extensive use of the Bayesian Theorem and sufficient creativity would be required.

According to [3] the aggregated numbers of above model asymptotically equal the solutions of the affine transformed pendulum equation for

- $S \rightarrow \infty$ (equivalent to $N \rightarrow \infty$ ) and

- $d t \rightarrow 0$.

Figure 2 shows a series of convergence-tests for different scaling parameters $S$ and time-step parameters $d t$. Blue and red lines show the aggregated results $X_{1}$ and $X_{2}$ of sample runs of the ABM. The black and green line show the scaled and translated analytic solutions $\overline{\varphi(t)}=$ $S(T+\cos (\sqrt{2} t))$ and $\overline{\psi(t)}=S(T-\sqrt{2} \sin (\sqrt{2} t))$ of the pendulum equation. For large time-steps sizes $d t$ the ABM model results becomes unstable similar to explicit 
Runge-Kutta methods for the solution of ODEs. Yet, the convergence properties can be verified.

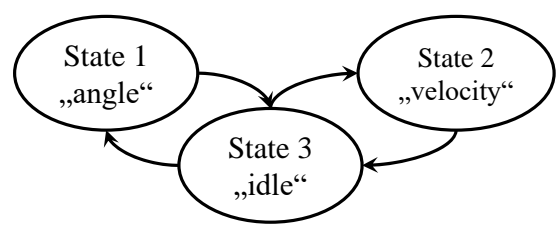

Figure 1: Flowchart of the agent-based model 1.

\section{Discussion}

The presented strategy leaves three interesting aspects for discussion.

Discussion of the Agent-Based Model. Clearly, the most interesting question concerning the derived model is, whether the model is not only a meta-model for the pendulum equation, but also a model for the real pendulum. According to Stachoviak [1] (compare with Section Introduction) it would be necessary to identify the agent-based model as a simplified image of a real pendulum.

Though undoubtedly consisting of microscopic elements like molecules or atoms, a pendulum (say, a rope pendulum with a sphere-shaped weight) is in principle not a system that is modelled as the sum of its microscopic elements. As the sphere is usually assumed to be made of a solid material, interactions between the elements are very limited and hardly contribute to the total movement of the object. Moreover, any molecule/atom in the swinging ball has an angle and a velocity and is not either "angle" or "velocity". If any, probably only a quantum-mechanic interpretation is somehow legitimate: Say one particle represents a fictive sub-atomic particle that is responsible for one quantum of angle and one quantum of velocity. If excited to either of the two states it results the object it is "attached" either to move or to accelerate. The chances for excitation and relaxation are directly linked to the laws of total energyconservation. Yet, as this interpretation is very vague, we would not really consider Model 1 as a model of the real pendulum, but solely as a meta-model.

Instead, we would suggest interpreting the derived model as a "numerical solver" of the pendulum equation. Using a microscopic solver on a macroscopic, equation based model is not unusual and is commonly
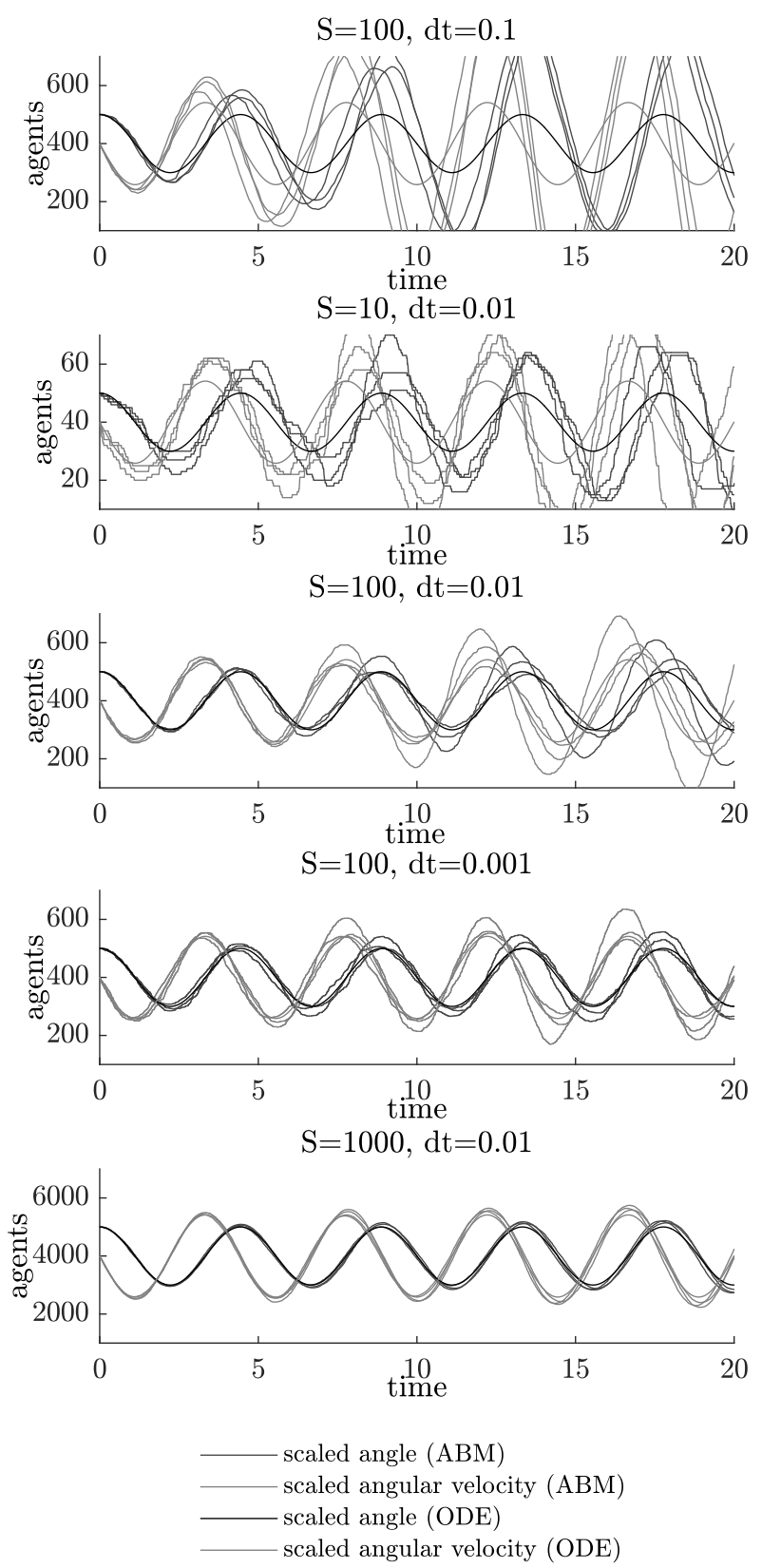

Figure 2: Tests for different values of $S$ and $d t$ for the stochastic ABM (3 runs each) defined in Model 1 for $\varphi_{0}=\frac{\pi}{2}, k=2$ and $T=4$. 
done e.g. in fluid dynamics: The macroscopic NavierStokes equations are "discretised" by particles on a grid (see HPP, FHP and Lattice-Boltzmann method [11]).

Discussion of the Benefits of the Model. The popularity of mentioned microscopic modelling approaches in fluid-dynamics simulation is mainly a result of their flexibility: Not only does a particle approach allow almost arbitrarily complex boundary-geometry (posing difficulties for a PDE approach with classic Navier-Stokes Equations), it can also be applied easily to simulate heterogeneous liquids. In general, microscopic models are clearly the most flexible of all model types with respect to model extensions and modifications due to their object-oriented description.

We observe similar flexibility for the microscopic pendulum model introducing a state-event: While stateevents are trivially detected in the microscopic approach (it is quantised) ODE solvers often need to iterate to find them adjusting their step-size.

Discussion of the Inverse Mean-Field Method. Apart from the derived model itself, the example of the pendulum model emphasises the generality of the inverse mean-field approximation for finding microscopic meta-models to macroscopic systems. Although there is no obvious reason for a microscopic representation of the system in that particular case the method definitely leads to a different point-of-view of the real system. R.E. Shannon, one of the founding-fathers of systems simulation, emphasised in 1975 that not only the measurable evaluation of various designs of a given system is target of a simulation model, but also the understanding of its behaviour [12]. Hence analysis of effects of different system components poses a vital contribution to any given research problem. The availability of a microscopic and a macroscopic model for one system allows two different points of view on the system components.

\section{Acknowledgement}

K-Projekt DEXHELPP is supported by BMVIT, BMWFW and the state of Vienna via COMET - Competence Centers for Excellent Technologies. Programme COMET is processed by FFG.

\section{References}

[1] Stachowiak H. Allgemeine Modelltheorie. Wien, New York: Springer-Verlag. 1973.

[2] Zeigler B. Why Should We Develop Simulation Models in Pairs? 2017.

URL https:

/ / www youtube.com/watch?v=iGXLxZcpUjA

[3] Bicher M. Classification of Microscopic Models with Respect to Aggregated System Behaviour. Dissertation, TU Wien, Vienna, Austria. 2017.

[4] Aoki M. Modeling aggregate behavior and fluctuations in economics: stochastic views of interacting agents. New York: Cambridge University Press. 2002.

[5] Deffuant G, Neau D, Amblard F, Weisbuch G. Mixing beliefs among interacting agents. Advances in Complex Systems. 2000;03(01n04):87-98.

[6] Webb SD, Keeling MJ, Boots M. Host-parasite interactions between the local and the mean-field: How and when does spatial population structure matter? Journal of Theoretical Biology. 2007;249(1):140-152.

[7] Benoit J, Nunes A, Telo da Gama M. Pair approximation models for disease spread. The European Physical Journal B. 2006;50(1-2):177-181.

[8] Morita S, Tainaka Ki. Undamped oscillations in prey-predator models on a finite size lattice. Population Ecology. 2006;48(2):99-105.

[9] Levins R. Some Demographic and Genetic Consequences of Environmental Heterogeneity for Biological Control. Bulletin of the Entomological Society of America. 1969;15(3):237-240.

[10] Kloeden PE. Numerical solution of stochastic differential equations. No. 23 in Applications of mathematics. Berlin ; New York: Springer, corr. 3rd print ed. 1999.

[11] Frisch U, Hasslacher B, Pomeau Y. Lattice-Gas Automata for the Navier-Stokes Equation. Physical Review Letters. 1986;56(14):1505-1508.

[12] Shannon RE. Systems Simulation: The Art and Science. Englewood Cliffs, N.J: Prentice-Hall. 1975. 


\title{
Spectral Analysis of Unevenly Spaced Data: Models and Application in Heart Rate Variability
}

\author{
Martin Bachler \\ Center for Health and Bioresources, AIT Austrian Institute of Technology, Giefinggasse 4, \\ 1210 Vienna, Austria; martin.bachler@ait.ac.at
}

SNE 27(4), 2017, 183-190, DOI: 10.11128/sne.27.tn.10393

Received: November 10, 2017, Revised: December 10, 2017,

Accepted: December 15, 2017

SNE - Simulation Notes Europe, ARGESIM Publisher Vienna,

ISSN Print 2305-9974, Online 2306-0271, www.sne-journal.org

Abstract. Heart rate variability (HRV) is the temporal variation of the interval between consecutive heartbeats. It can be analyzed by numerous methods, including spectral analysis. However, HRV time series naturally consist of unevenly spaced data. Several methods emerged to counteract this problem, usually yielding different results. Therefore, in this work three spectral analysis methods were investigated: the Welch, Lomb-Scargle, and Burg method. Their properties were analyzed by theoretical considerations and verified in simulations. Using an oscillator network model with the integral pulse frequency modulation model, artificial HRV time series were generated. Their power spectral densities and their dominant frequencies were evaluated and compared to the nominal values of the simulated time series. The results of these experiments and the theoretical considerations suggest that the Lomb-Scargle method is the most exact in reproducing power spectral densities and dominant frequencies of unevenly spaced HRV data.

\section{Introduction}

Heart rate variability (HRV) is the temporal variation of the interval between consecutive heartbeats. It reflects the ability of an organism to change the frequency of the cardiac rhythm on a beat-to-beat basis.

The heart rhythm is controlled via the autonomic nervous system, whereby its two branches, the sympathetic and the parasympathetic, produce acceleration or deceleration. The sympathetic nervous system has an activating function and causes an increase in the heart rate, the parasympathetic nervous system leads to an inhibition of the activity and therefore a decrease in the heart rate [1].

The higher the heart rate variability, the faster and more flexible the organism can adapt to internal and external influences, by optimizing the interplay of the sympathetic and parasympathetic nervous system. Physical or psychological stress usually results in an increase in the heart rate, which then recedes during relief and relaxation. Under chronic stress, however, this adaptability is restricted and consequently the HRV is reduced due to the constantly high tension [2]. The regulatory system of the heart rate has several highly interconnected in- and outputs, which lead to complex irregularity in the resulting heart rate. Therefore, it is reasonable to hypothesize that a defect in the regulatory system leads to a decreased irregularity of the heart rate. Thus, the analysis of the HRV strives to quantify the amount of variability and irregularity.

In diagnostic terms, HRV is not a clinical parameter or biomarker, but an umbrella term. It can be quantified from a series of RR intervals (i.e., the series of beat-to-beat intervals) by numerous parameters in three categories:

- By statistical parameters in the time-domain: the beat-to-beat heart rate is interpreted as a time series and is described using common methods of time series analysis [1].

- By spectral analysis in the frequency-domain: the beat-to-beat heart rate is regarded as a timedependent function and transformed into the frequency-domain. The power spectral density of certain, standardized frequency bands reflects various physiological processes [1].

- By non-linear analysis methods: This collective term encompasses all methods that attempt to 
quantify the complex dynamics of the physiological interactions of the cardiac, circulatory and nervous system to control the heart rate. Methods from the field of chaos theory may be applied to determine short-term variability (Poincaré plot) or the degree of irregularity (entropy analysis) [3, 4].

The analysis of the power spectral density (PSD) provides information on how power distributes as a function of frequency. The power of certain frequency bands can be linked to sympathetic and parasympathetic activity, respiratory sinus arrhythmia, circadian rhythms, and further physiologic influences on the HRV. The Guidelines on Heart Rate Variability distinguish between the total power of the whole spectrum and the spectral power of four separated frequency bands, usually determined in $m s^{2}$, as summarized in table 1 .

\begin{tabular}{c|l|c}
\hline Name & Description & Frequency range \\
\hline TP & Total Power & $0-0.4 \mathrm{~Hz}$ \\
\hline ULF & Ultra low frequency & $0-0.003 \mathrm{~Hz}$ \\
\hline VLF & Very low frequency & $0.003-0.04 \mathrm{~Hz}$ \\
\hline LF & Low frequency & $0.04-0.15 \mathrm{~Hz}$ \\
\hline HF & High frequency & $0.15-0.4 \mathrm{~Hz}$ \\
\hline
\end{tabular}

Table 1: Summary of frequency-domain measures of HRV.

The applicability of these measures depends on the duration of the recording. Power in the ultra low frequency (ULF) and very low frequency (VLF) ranges can only be determined in long-term $24 \mathrm{~h}$ recordings, since their cycle times $(1 / f)$ exceed 5 minutes. On the other hand, the power in the low (LF) and high (HF) frequency ranges can be calculated in short- and long-term recordings alike. However, since the heart rate modulations during long-term recordings exhibit lower stability, LF and HF become less easily interpretable. The total power (TP) is applicable in short- and long-term recordings without constraint [1]. Figure 1 shows an example of a PSD analysis with frequency bands.

The estimation of the PSD itself is not a trivial process, since methods for spectral analysis usually require evenly sampled discrete time series. This is not the case for a series of RR intervals, since the heartbeats form a discrete event series following a stochastic process. Several methods emerged to counteract this problem, essentially based on two approaches. Non-parametric methods do not assume a functional form of the PSD a

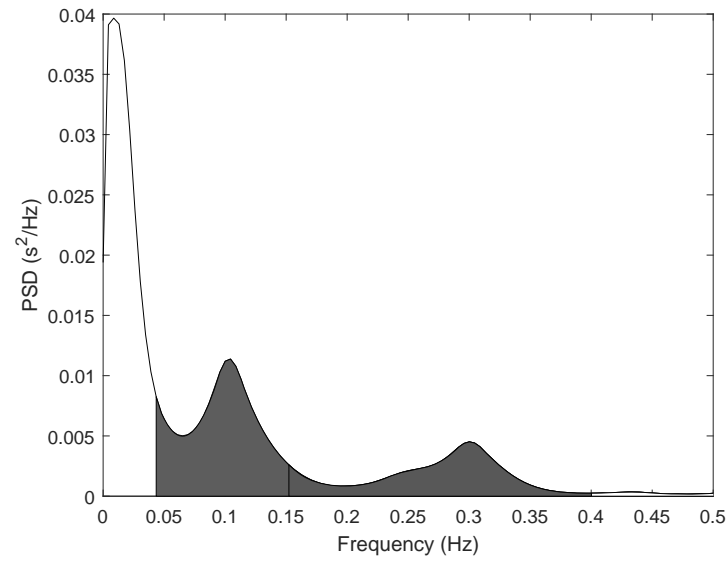

Figure 1: Example of a power spectral density estimate of a 5 minute recording. The blue area marks the LF, the red area the HF component of the spectrum.

priori, while for parametric methods, assumptions are made for the signal which lead to a parametric, functional form of the PSD. In the latter case, parameters for the resulting model have to be determined.

Both approaches have advantages and disadvantages. Therefore, in this work, popular models for the PSD estimation of HRV time series are investigated by theoretical considerations and application of the methods to simulated data.

\section{Methods}

In the first part of this section, the non-parametric and parametric PSD estimation methods commonly used in HRV analysis are described. In the second part, methods to simulate artificial HRV time series are presented. The PSD estimation methods are then applied to the simulated time series and their results compared to the nominal values of the simulation.

\subsection{Non-Parametric PSD Methods}

Non-parametric methods are based on the fast Fourier transform. Due to finite data length the definition of the PSD is modified to an empirical version based on the discrete-time Fourier transform (DTFT) and thus an estimate. This estimate is referred to as a periodogram and is calculated for a signal $y(t)$ as follows: 


$$
\hat{S}_{X X}(\omega)=\frac{1}{N}\left|\sum_{t=1}^{N} y(t) e^{-i \omega t}\right|^{2} .
$$

However, this direct DTFT approach has high variance and is not consistent [5]. Furthermore, these estimates exhibit distortions due to spectral leakage caused by the windowing effect of finite data lengths, which may cause a superposition of weak parts of the signal [2]. Therefore, methods such as the Welch method were developed to reduce variance and spectral leakage at the expense of the resolution [5].

Welch Method In the Welch method, the data points are divided into segments that can overlap. The data points of each segment are weighted with a window function before calculating the periodogram. In order to obtain the estimated value of the PSD, the periodograms of the individual segments are averaged. This averaging should lead to a reduction in variance [6].

In order to create an evenly sampled discrete time series, the RR intervals in this work are first interpolated at $7 \mathrm{~Hz}$ and subsequently evaluated using the Welch method with adjacent segments overlapping at $50 \%$ and a Hamming window function [7].

Lomb-Scargle Method An advancement of the classical periodogram is the variant of Lomb and Scargle $[8,9]$, whose great advantage is the applicability to stochastic, irregularly sampled data. It was originally developed for application in astronomical research, where observation periods are limited and irregular due to planetary constellations and weather influences. It is able to find weak periodic signals in otherwise random, unevenly sampled data. Studies show that the Lomb-Scargle periodogram reduces possible distortions or erroneous results that can result from any form of interpolation [10].

Consider $x_{k}$ being the RR intervals and $t_{k}$ derived from the discrete event series of $\mathrm{R}$ peaks, where $k=$ $1, \ldots, N$. The Lomb-Scargle periodogram is defined by [8] as

$$
\begin{aligned}
& P_{L S}(\omega)= \frac{1}{2 \sigma^{2}}\left\{\frac{\left[\sum_{k=1}^{N}\left(x_{k}-\bar{x}\right) \cos \left(\omega\left(t_{k}-\tau\right)\right)\right]^{2}}{\sum_{k=1}^{N} \cos ^{2}\left(\omega\left(t_{k}-\tau\right)\right)}+\right. \\
&\left.\frac{\left[\sum_{k=1}^{N}\left(x_{k}-\bar{x}\right) \sin \left(\omega\left(t_{k}-\tau\right)\right)\right]^{2}}{\sum_{k=1}^{N} \sin ^{2}\left(\omega\left(t_{k}-\tau\right)\right)}\right\},
\end{aligned}
$$

where $\bar{x}$ is the mean and $\sigma^{2}$ the variance of $x$. For every angular frequency $\omega=2 \pi f$ a time offset $\tau$ is chosen as

$$
\tan (2 \omega \tau)=\frac{\sum_{k=1}^{N} \sin \left(2 \omega t_{k}\right)}{\sum_{k=1}^{N} \cos \left(2 \omega t_{k}\right)} .
$$

The time offset $\tau$ guarantees the time invariance of $P_{L S}$, since every shift of $t_{k}$ results in an equivalent shift in the offset.

\subsection{Parametric PSD Methods}

Non-parametric methods do not assume any conditions for the signal except for stationarity. In the case of a parametric or model-based approach for spectral estimation, the signal is given a certain functional form and thus a model is created. The parameters of the model must be determined.

Burg Method Despite the existence of a wide variety of parametric methods, the most wide spread for analysis of HRV models the RR intervals as an autoregressive process of the form

$$
y(t)=c+\sum_{i=1}^{p} \phi_{i} y(t-i)+\varepsilon_{t},
$$

where $c$ is a constant, $\varepsilon_{t}$ a white-noise error term, and $\phi_{i}$ represents the model parameters. The order $p$ of the model influences its properties: the lower the order, the smoother the resulting PSD function. Previous studies suggest that an order of $p=16$ is best suited for application in HRV analysis [11, 2]. Finally, the Burg method, whose algorithm minimizes predefined forward and backward prediction errors, is used to estimate the model parameters $\phi_{i}$ [12].

The three methods for PSD estimation are compared in figure 2. Due to the segmentation of the input series, the Welch method shows fewer resulting data points than the other two methods. The Lomb-Scargle method exhibits more distinct peaks and valleys with maximum values about twice the height of the other two methods. However, the integral of the power in the LF and HF band is comparable especially to the Burg method. The Burg method, due to the underlying autoregressive model, shows the smoothest shape. 

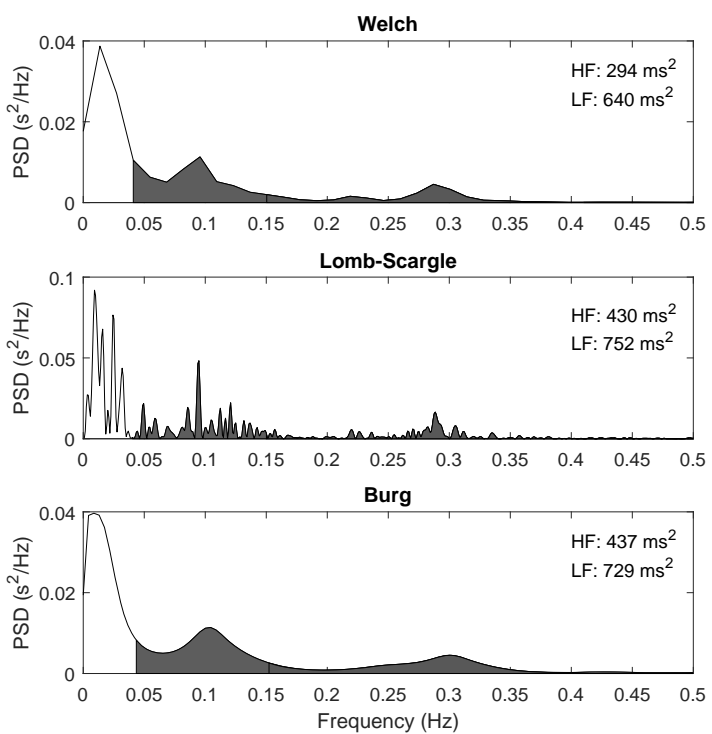

Figure 2: Comparison of the three PSD estimation methods of the same HRV time series: Welch (50\% overlap, Hamming window), Lomb-Scargle, and Burg (order 16).

\subsection{Simulation of HRV Time Series}

Different frequency bands in the HRV time series reflect different physiological feedback mechanisms. In order to generate artificial HRV time series it is possible to reversely approximate this connection. In this work, the following two models were coupled in order to simulate unevenly spaced HRV data.

Oscillator Network Model Based on the work of Brennan et al. [13], an interconnected network of sine oscillators is created to simulate the cardiac control system, as shown in figure 3. To create a more realistic frequency distribution, each frequency band (VLF, LF, and HF) is simulated by three sub-oscillators. Thereby, each sub-oscillator is determined by its fundamental frequency $f_{i}$ (randomly chosen in the respective frequency range) and its amplitude $a_{i}$, randomly chosen in the range of $[20,40] \mathrm{ms}$. Finally, the cardiac control system $m(t)$ is given by

$$
\begin{aligned}
s_{V L F}(t) & =\sum_{i=1}^{3} a_{V L F i} \cdot \sin \left(2 \pi f_{V L F i} t\right) \\
s_{L F}(t) & =\sum_{i=1}^{3} a_{L F i} \cdot \sin \left(2 \pi f_{L F i} t\right)
\end{aligned}
$$

$$
\begin{aligned}
s_{H F}(t) & =\sum_{i=1}^{3} a_{H F i} \cdot \sin \left(2 \pi f_{H F i} t\right) \\
m(t) & =H R+s_{V L F}+s_{L F}+s_{H F},
\end{aligned}
$$

where $H R$ is a constant offset representing the average heart rate (HR).

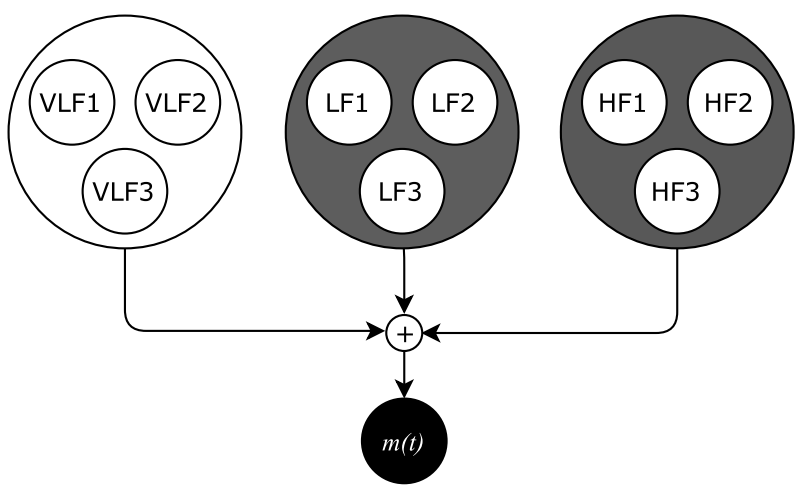

Figure 3: Network of oscillators to simulate the cardiac control system $m(t)$.

Integral Pulse Frequency Modulation Model Since equation 7 produces a continuous model of the cardiac control system, another step is required to generate unevenly spaced HRV data. In this step, the integral pulse frequency modulation (IPFM) model is applied [14]. It generates the occurrence times of heartbeats by integrating the input signal $m(t)$ until it reaches unity. This point in time is then considered as occurrence of one heartbeat, and the integrator is reset to zero. It is represented as

$$
1=\int_{t_{k}}^{t_{k+1}} m(t) d t,
$$

where $t_{k}$ denotes the occurrence times of heartbeats. In this work, equation 9 was solved numerically for $t \in[0,300]$ seconds. This duration corresponds to HRV measurements of 5 minutes and follows the Guidelines on Heart Rate Variability [1]. Finally, the HRV time series consists of the intervals between consecutive heartbeats, the $R R$ intervals, and is therefore derived from the $t_{k}$ series as

$$
R R_{k}=t_{k+1}-t_{k}
$$




\subsection{Model Comparison}

Since the HRV data in this work is generated artificially, it is possible to determine their nominal values and compare them to the results of the investigated methods. This comparison will focus on two features of the HRV time series: (1) the power spectral density and (2) the most dominant frequency. Both features will be independently evaluated for the LF and HF component.

Power Spectral Density The PSD of a given continuous signal $x(t)$ in the time domain is given by

$$
P=\lim _{T \rightarrow \infty} \frac{1}{2 T} \int_{-T}^{T}|x(t)|^{2} d t .
$$

For a signal of finite duration $T$, equation 11 reduces to the variance of the signal. Since the frequency components are generated separately in the oscillator model, the nominal values for LF and HF power $p_{L F}$ and $p_{H F}$ are calculated by

$$
\begin{aligned}
P_{L F} & =\operatorname{Var}\left(s_{L F}\right), \text { and } \\
P_{H F} & =\operatorname{Var}\left(s_{H F}\right) .
\end{aligned}
$$

As comparison, the band limited PSD of the Welch, Lomb-Scargle, and Burg method will be calculated by

$$
\hat{P}=2 \int_{f_{1}}^{f_{2}} \hat{S}_{X X}(2 \pi f) d f
$$

from their respective PSD estimations $\hat{S}_{X X}$ and in the frequency ranges $\left[f_{1}, f_{2}\right]$ according to table 1 .

Dominant Frequency Again, the fact that the frequency components are generated separately is used to obtain nominal values for the model comparison. For the component $s_{L F}$ (and likewise for $s_{H F}$ ), the dominant frequency $f_{d}$ is determined by the amplitude:

$$
\begin{aligned}
i & =\underset{i}{\operatorname{argmax}}\left(a_{L F i}\right), \\
f_{d} & =f_{L F i} .
\end{aligned}
$$

The comparative values from the PSD methods are obtained from the PSD estimates $\hat{S}_{X X}$ by

$$
\hat{f}_{d}=\underset{f \in\left[f_{1}, f_{2}\right]}{\operatorname{argmax}}\left(\hat{S}_{X X}(2 \pi f)\right) .
$$

\section{Results}

One thousand simulation runs of the HRV model were performed to gather nominal values and approximations by the Welch, the Lomb-Scargle, and the Burg PSD estimation methods. Their results are reported in this section.

\subsection{Power Spectral Density}

Figure 4 summarizes the differences between the PSD evaluated by the three methods under investigation and the nominal values as boxplots. The same results are aggregated in table 2 as mean (standard deviation). While in the LF band, the Welch method shows the best result regarding the mean and median differences, it also shows the worst in the HF band. In contrast, the LombScargle method exhibits the worst result regarding the mean and median differences in the LF range. However, its scattering is by far the lowest in all tested cases, suggesting a higher consistency compared to the other methods.

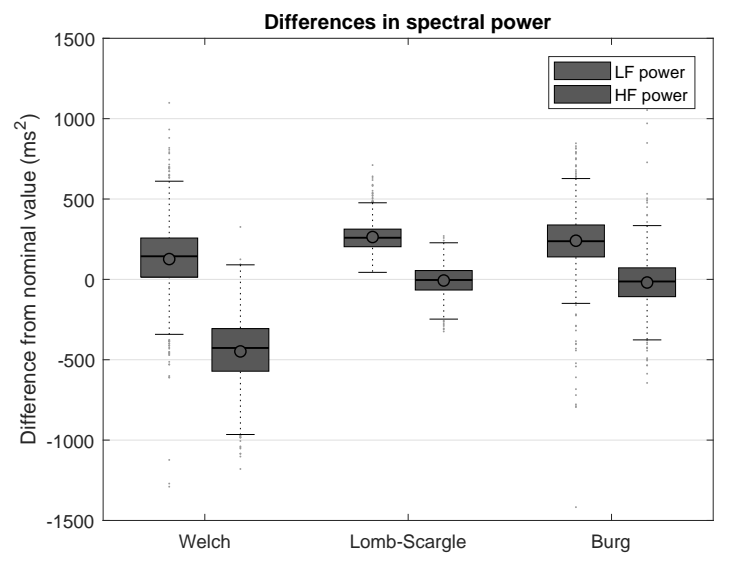

Figure 4: Deviation of the power spectral density approximations from the nominal values in the low- and high frequency (LF and HF) range.

Figure 5 shows the individual differences of all simulation runs as a function of the nominal values. It allows the interpretation of the results regarding systematic errors. Again, the lower dispersion of the LombScargle method is clearly visible. Furthermore, all methods display a dependency on the nominal values: the higher the nominal values, the higher the dispersion of the differences. Lastly, trends are visible in the evaluation of the LF range by the Lomb-Scargle method and 

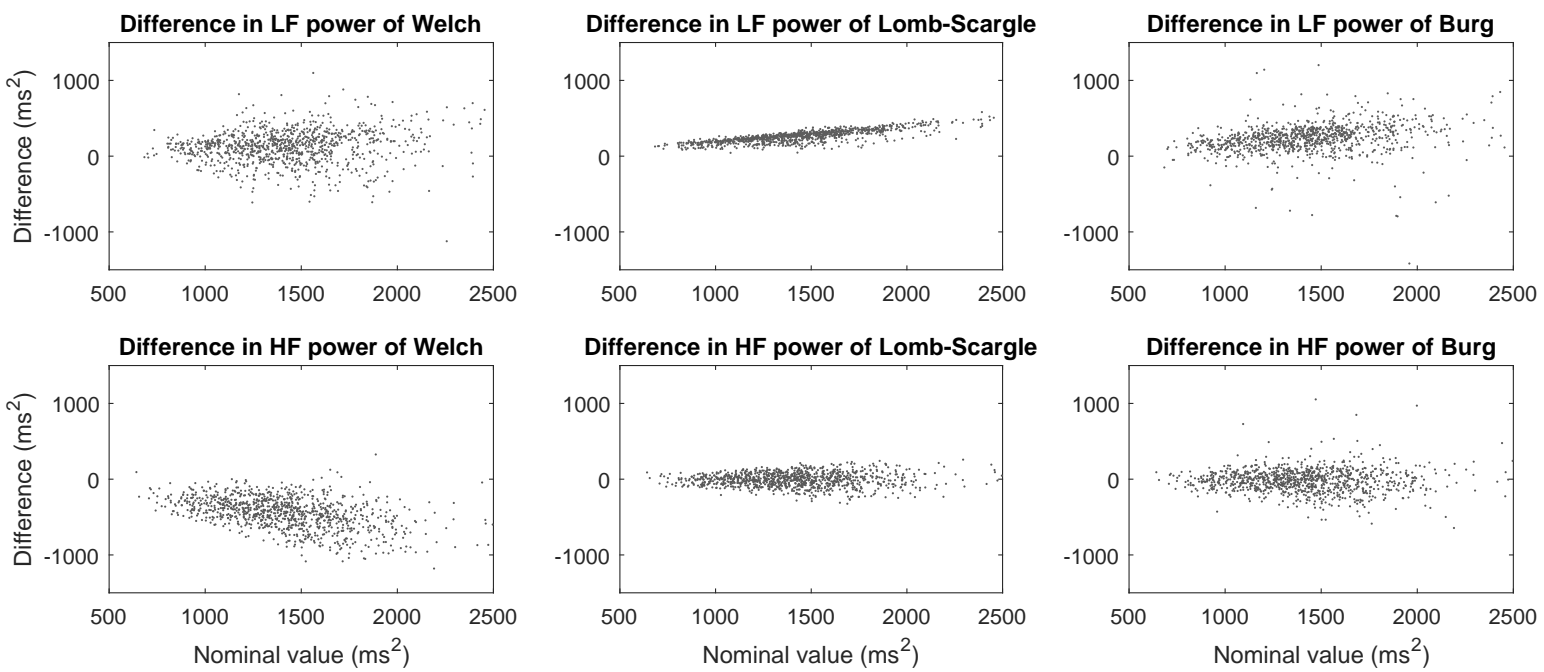

Figure 5: Deviation of the power spectral density approximations from the nominal values, shown as a function of the nominal values and separated by method and frequency range.

\begin{tabular}{c|c|c}
\hline Power Spectral Density & LF $\left(m s^{2}\right)$ & HF $\left(m s^{2}\right)$ \\
\hline Welch & $126(232)$ & $-448(214)$ \\
\hline Lomb-Scargle & $264(88)$ & $-7(93)$ \\
\hline Burg & $241(281)$ & $-19(156)$ \\
\hline
\end{tabular}

Table 2: Deviation of the power spectral density approximations from the nominal values in the lowand high frequency (LF and HF) range, stated as mean (Standard deviation).

the HF range by the Welch method. In these cases, apparently the differences of the results and nominal values depend on the actual power of the respective frequency band.

\subsection{Dominant Frequency}

Figure 6 reports the deviation of the detected dominant frequencies from the nominal values. Since the results are not normally distributed, table 3 summarizes them as median [1st quartile, 3rd quartile]. In contrast to the results of the PSD, the Lomb-Scargle method outperforms the other methods regarding the dominant frequency in all cases. Again, a low scattering suggests a high consistency of this method.

Figure 7 reports the individual results as a function of the nominal values. All results exhibit the interesting pattern that differences are positive for low frequency

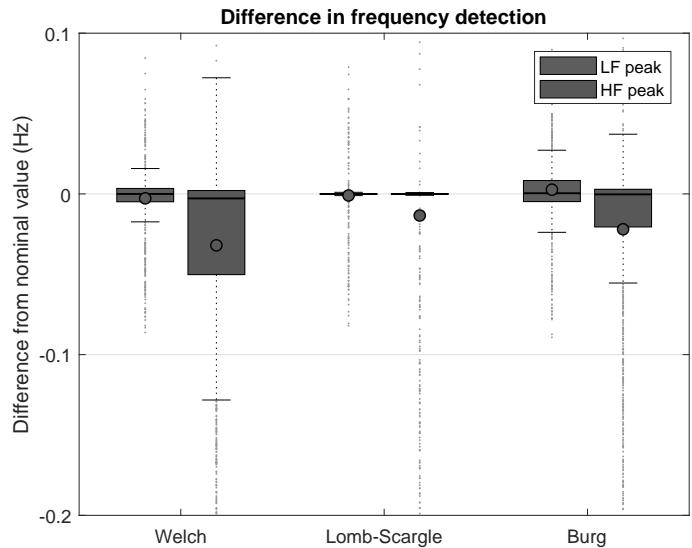

Figure 6: Deviation of the detected dominant frequency from the nominal values in the low- and high frequency (LF and HF) range.

values, and negative for high frequency values. This points out the systematic error that measurement results are too high for low frequencies, and too low for high frequencies. In other words, all three methods exhibit a trend towards the center of the frequency range when looking for the most dominant frequency. Apart from this common systematic error, the figure mirrors the results of figure 6 and table 3 regarding the dispersion of the differences. Finally, a reduction of frequency resolution in the Welch method becomes obvious as recurring pattern around the zero difference. 

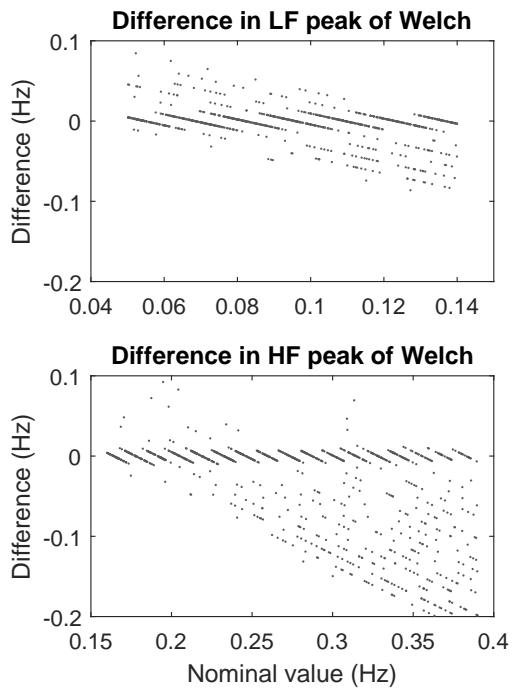
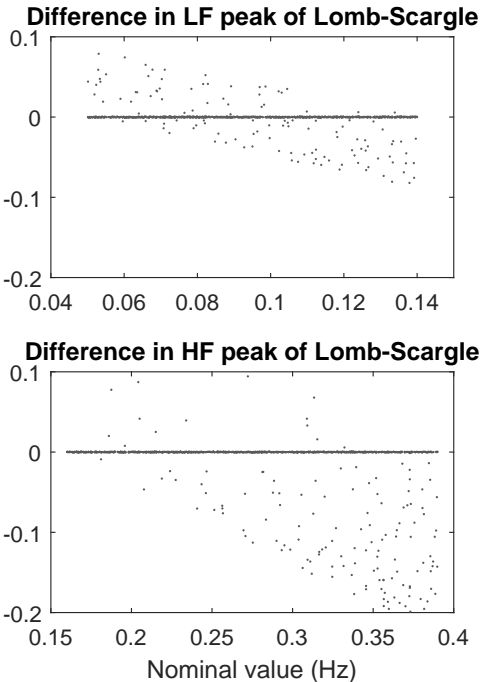
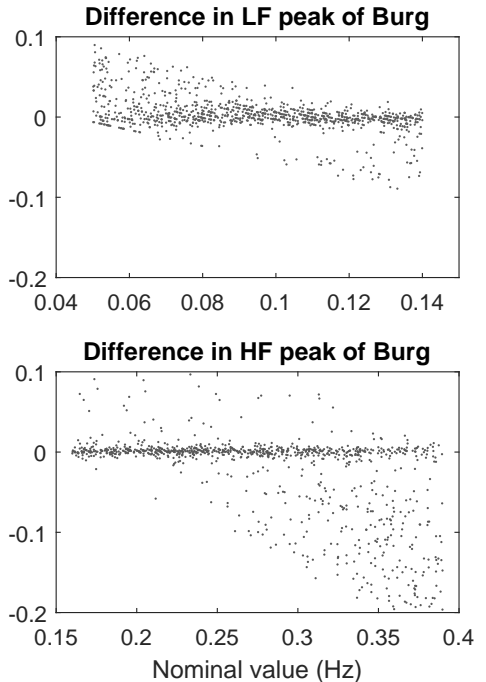

Figure 7: Deviation of the detected dominant frequency peak from the nominal values, shown as a function of the nominal values and separated by method and frequency range. A positive difference results from measurement values higher than the nominal values.

\begin{tabular}{c|c|c}
\hline Dominant Freq. & LF $(m H z)$ & HF $(m H z)$ \\
\hline Welch & $0.0[-4.9,3.4]$ & $-2.8[-50.2,2.1]$ \\
\hline Lomb-Scargle & $0.0[-0.3,0.2]$ & $-0.1[-0.3,0.2]$ \\
\hline Burg & $0.4[-4.8,8.3]$ & $-0.3[-20.62 .9]$ \\
\hline
\end{tabular}

Table 3: Deviation of the detected dominant frequency from the nominal values in the low- and high frequency (LF and HF) range. Since the data is not normally distributed, they are stated as median [1st quartile, 3rd quartile].

\section{Discussion}

From a clinical point of view, the calculation of the HRV can be used to assess the state of the autonomous nervous system to evaluate e.g. stress, depression, or brain damage [2]. Regarding cardiovascular diseases, HRV analysis aids in the diagnosis and risk stratification of numerous pathologies. Alterations in certain HRV measures during or after myocardial infarction, in congestive heart failure, or in diabetic neuropathy have been linked to increased mortality [1].

While the relationship between the frequency components HF, LF, and VLF and the activity of the nervous system as well as other regulatory mechanisms is undoubted [2], the selection of the proper method to estimate the PSD and their parameters (for parametric methods) remains controversial. Studies showed that the power calculated in the respective frequency bands varies significantly between methods [15].

The Welch method requires evenly sampled data points, which necessitates resampling of the time series. The choice of the resampling method and frequency therefore will influence the PSD estimate. Besides, due to windowing and averaging, the Welch method suffers from a loss in frequency resolution. However, since HRV is usually quantified using the standardized and rather broad frequency bands, this might not be an issue. Furthermore, the windowing and averaging leads to a better statistical stability regarding outliers. Therefore, a small amount of artifacts or ectopic beats in the interval time series is tolerable. $[1,2,15]$

While the Burg methods differs from the Welch method in being parametric instead of non-parametric, they share several advantages and disadvantages. It requires the choice of an order, which affects the result. However, several studies investigated this topic and reached the conclusion, that an order of 16 is generally acceptable in HRV analysis [11,2]. Similar to the Welch method, the autoregressive model leads to a smoothing effect, limiting the frequency resolution but allowing for some outliers in the time series [15].

The Lomb-Scargle method can be considered the most exact method, since there is no need for resampling (and thus, an alteration of the input data itself) 
nor for the choice of parameters. However, it is rarely used in medical studies, probably for the lack of being mentioned in guidelines [1] or thorough reviews [2]. Furthermore, due to the lack of any smoothing, outliers may easily disrupt the PSD estimate, especially the HF component. Therefore, the Lomb-Scargle method requires the most thorough filtering of RR intervals.

Apart from the theoretic analysis, the PSD estimation methods investigated in this work were applied to artificially simulated HRV data with known properties. The results of these model comparisons clearly point towards the Lomb-Scargle method as the most exact method. It showed the best capability to consistently reproduce the power spectral density as well as the accurate detection of the most dominant frequencies. The findings of these simulations are in line with the theoretical considerations.

\section{Conclusion}

In this work, three methods for spectral analysis of HRV time series were investigated theoretically and using simulated HRV data. The experimental results as well as the theoretical considerations suggest that the LombScargle method is the most exact. However, it must be assumed that it is also the most vulnerable to outliers in the HRV time series. Therefore, the Lomb-Scargle PSD is only recommended after thorough filtering of the RR intervals, if an error-free time series can be assumed. The effects of outliers on PSD estimation methods therefore will be investigated in future works.

\section{References}

[1] American Heart Association Inc; European Society of Cardiology. Guidelines - Heart rate variability. European Heart Journal. 1996;17:354-381.

[2] Rajendra Acharya U, Paul Joseph K, Kannathal N, Lim C, Suri J. Heart rate variability: a review. Medical and Biological Engineering and Computing. 2006; 44:1031-1051.

[3] Bachler M, Hörtenhuber M, Mayer C, Holzinger A, Wassertheurer S. Entropy-Based Data Mining on the Example of Cardiac Arrhythmia Suppression. In: Brain Informatics and Health, edited by Ślęzak D, Tan AH, Peters J, Schwabe L, vol. 8609 of Lecture Notes in Computer Science, pp. 574-585. Springer International Publishing. 2014;ISBN: 978-3-319-09890-6 (Print) 978-3-319-09891-3 (Online).
[4] Hagmair S, Bachler M, Wassertheurer S, Mayer C. Nonlinear Methods in Heart Rate Variability: Can they Distinguish between Nonpathological and Pathological Subjects? SNE Simulation Notes Europe. 2015; 25(3-4):145-150.

[5] Stoica P, Moses RL, et al. Spectral analysis of signals, vol. 452. Pearson Prentice Hall Upper Saddle River, NJ. 2005.

[6] Welch P. The use of fast Fourier transform for the estimation of power spectra: a method based on time averaging over short, modified periodograms. IEEE Transactions on audio and electroacoustics. 1967; 15(2):70-73.

[7] Clifford G, Tarassenko L. Quantifying errors in spectral estimates of HRV due to beat replacement and resampling. Biomedical Engineering, IEEE Transactions on. 2005;52(4):630-638.

[8] Lomb N. Least-squares frequency analysis of unequally spaced data. Astrophysics and space science. 1976; 39(2):447-462.

[9] Scargle JD. Studies in astronomical time series analysis. II-Statistical aspects of spectral analysis of unevenly spaced data. The Astrophysical Journal. 1982; 263:835-853.

[10] Ruf T. The Lomb-Scargle periodogram in biological rhythm research: analysis of incomplete and unequally spaced time-series. Biological Rhythm Research. 1999; 30(2):178-201.

[11] Boardman A, Schlindwein FS, Rocha AP, Leite A. A study on the optimum order of autoregressive models for heart rate variability. Physiological Measurement. 2002;23(2):325.

[12] Burg JP. A new analysis technique for time series data. In: Modern Spectrum Analysis, edited by Childers DG. New York: IEEE Press. 1968;.

[13] Brennan M, Palaniswami M, Kamen P. Poincaré plot interpretation using a physiological model of HRV based on a network of oscillators. American Journal of Physiology - Heart and Circulatory Physiology. 2002; 283(5):H1873-H1886.

[14] Solem K, Laguna P, Sornmo L. An efficient method for handling ectopic beats using the heart timing signal. Biomedical Engineering, IEEE Transactions on. 2006; 53(1):13-20.

[15] Estévez M, Machado C, Leisman G, Estévez-Hernández T, Arias-Morales A, Machado A, Montes-Brown J. Spectral analysis of heart rate variability. International Journal on Disability and Human Development. 2016; 15(1):5-17. 


\title{
Pathways of Migrants and Refugees - a Simulation Approach
}

\author{
Felix Breitenecker ${ }^{1}$, Tamara Vobruba ${ }^{1}$, Andreas Körner ${ }^{1}$, Nikolas Popper ${ }^{2}$
}

${ }^{1}$ TU Wien, Inst. f. Analysis und Scientific Computing, Mathematical Modelling Group,

Wiedner Hauptstrasse 8-10, 1040 Vienna, Austria

${ }^{2}$ dwh Simulation Services, Neustiftgasse 57-59, 1070 Vienna, Austria; *felix.breitenecker@tuwien.ac.at

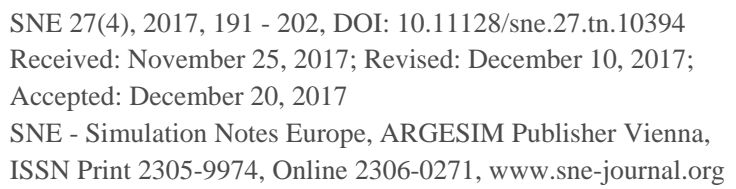

SNE 27(4), 2017, 191 - 202, DOI: 10.11128/sne.27.tn.10394 Received: November 25, 2017; Revised: December 10, 2017; Accepted: December 20, 2017

SNE - Simulation Notes Europe, ARGESIM Publisher Vienna, ISSN Print 2305-9974, Online 2306-0271, www.sne-journal.org

Abstract. In autumn 2015, Europe was confronted with a dramatic migration and refugee's movement - the so-called Refugee Crisis 2015. No ebbing of the migration and refugee pathways can be expected for the near future. Can simulation help to analyse the pathway of migration in such a critical case, and can simulation forecast pathways of migrants and refugees under different circumstances? This contribution first sketches variants of the Spatial Interaction Model, a model approach for any kind of population shift in certain regions. This more theoretical introduction is followed by the development of a Spatial Interaction Model for the pathway of migrants and refugees during the Refugee Crisis 2015, and by model implementation and identification of model parameters based on UNHCR data reported during the crisis.
Next follows a spatial extension including the Mediterranean Sea route allowing a forecast and real data comparison for pathways of migrants and refugees in summer 2016. Last step is development of a Scenario Model allowing the study of possible control actions. The simulation model does not provide direct help for the people involved, but the simulation can help in better understanding and improving the situation of migrants and refugees - the authors hope.

\section{Introduction}

In September and October 2015 a wave of refugees shocked Europe - later on called Refugee Crisis 2015. UNHCR reported daily dramatic data - refugees on route from Syria to western Europe, mainly on transit in Eastern Europe, and applying for asylum in Western Europe. Figure 1 shows two snapshots from animations of UNHCR data- the one from September 5, 2015, when the wave started, and the other from October 30, 2015, when the wave ebbed down.
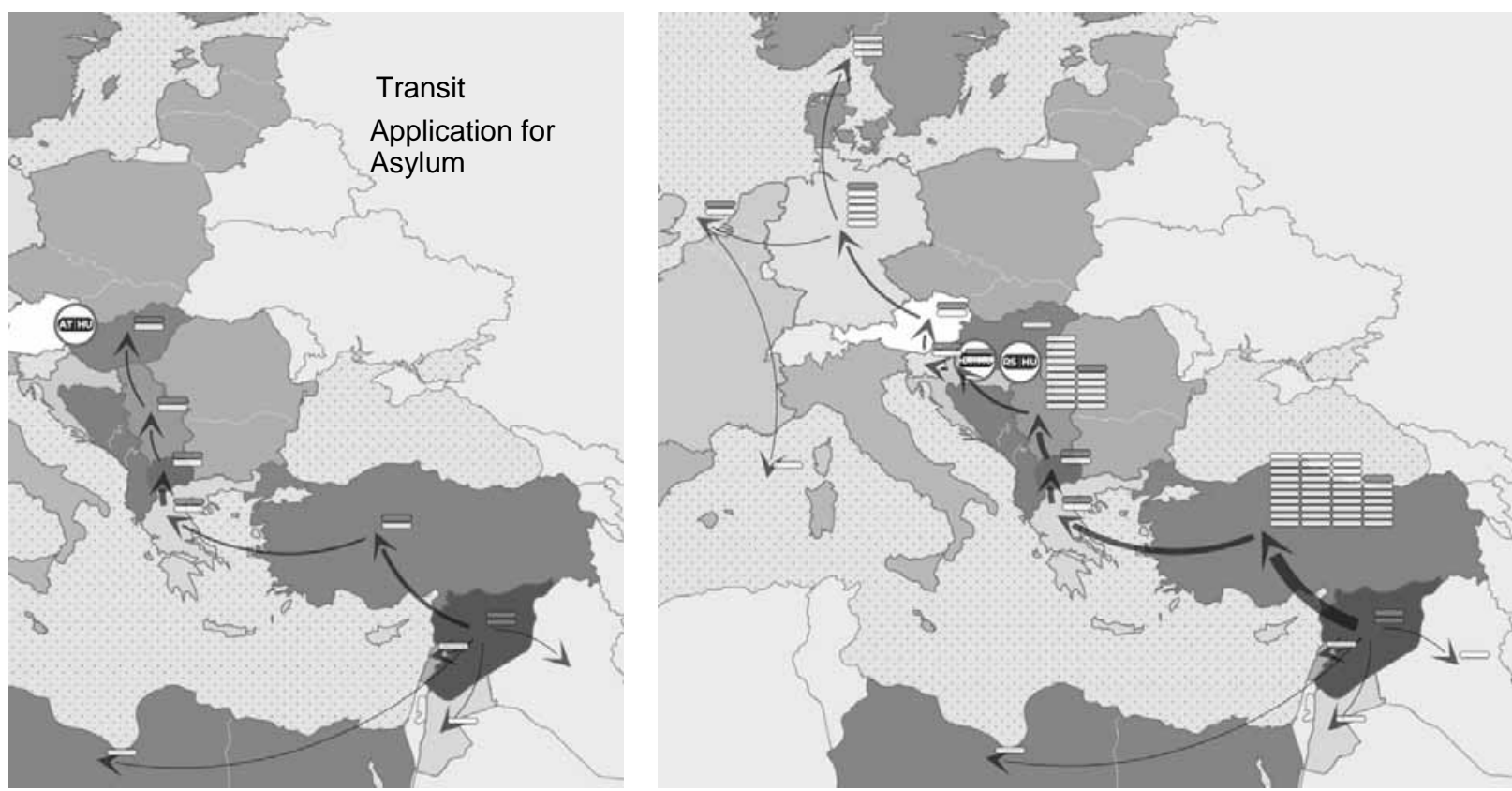

Figure 1. Data animation of refugees' pathway in Refugee Crisis 2015 - snapshot 2015-09-05 (left) and 2015-10-30 (right). 
The data animation of this specific refugee wave (two snapshots in Figure 1) was performed by $d w h$ Simulation Services for ORF 1 Online (Austrian Broadcast Company, Science Program Online). The Mathematical Modelling Group of the Institute for Analysis and Scientific Computing at TU Wien is closely co-operating with $d w h$ Simulation Services, and discussions on the Refugee Crisis 2015 initiated a small project for trying to develop a simulation model for this specific refugee wave, with possibilities to parametrize for other possible pathways of refugees or migrants.

First step was the interpretation of a refugee wave as a special case of migration behaviour. Migration behaviour seems to be driven by individual and personal circumstances of the refugees. Moreover, it becomes clear from a macroscopic viewpoint, that migration is a special kind of spatial population dynamics. Parts of a population migrate from one region into another region, driven by repulsive forces in the start region, and by attractive forces of the destination region(s). Regions often try to control migration by border control - separation forces for the migration movement. These forces, together with the pathway of migration, allow a modelling approach for the migration movement as behavioural spatial interaction model.

The pathways of migrants and refugees can be interpreted as spacial interaction of parts of populations in certain regions. The so-called Spatial Interaction Model is a macroscopic model approach for describing any kind of spatial interaction behaviour between populations or regions (described in Section 1 Spatial Interaction Models). This modelling approach has a wide range of applications, from traffic flows, movement of commuters or migrants, trade of goods or transmission of messages - and therefore seems to be appropriate for modelling also the pathway of migrants and refugees.

The best known and widely used type of Spatial Interaction Models is the Social Gravity Model. (sketched in Section 2 Social Gravity Models). The basic idea is the description of the interaction between populations or regions with a relation referring to Newton's law of gravity.

Section 3 Migration Pathway Model develops a network of generalized social gravity models for the pathway of migrants and refugees, taking into account special demands with respect to regions of origin, possible regions of destination and the transit regions, and with respect to the heterogeneity of the attraction or repulsion of these regions influencing the migration behaviour. It turns out that it is necessary to introduce transit countries, characterized by an attraction not from the country itself, but by the attraction of following countries.

Indeed, in spring 2016, the developed model could be parametrized and identified for the refugee wave of the Refugee Crisis 2015 - with specific submodels for the attracting and repelling attributes of the involved countries, and with data from UNHCR (Section 4 Refugee Crisis Model).

Obviously, the Refugee Crisis 2015 was not the only refugee wave. In general, no ebbing of the migration and refugee pathways can be expected, and the challenge was, whether the developed model is able to forecast the refugee wave expected for June 2016. Section 5 Forecast Model describes the necessary model changes - close down of Balkan route and opening of Mediterranean route - and a successful forecast.

It turns out, that the model - first published and presented in autumn 2016 [7, 8] is 'general' enough to study qualitatively also certain possible control strategies, proposed by politicians, with more or less careful considerations - close-down of certain specific borders, etc. briefly sketched in Section 6 Scenario Model.

A summary in Section 7 tries to evaluate the developed model. Main conclusions is: Models are in any case a simplification of reality, but they should help in better understanding of complex dynamics as migration movement, and the intention of this model is to show strategies to improve the situation of migrant population and resident population under appropriate prerequisites.

\section{Spatial Interaction Models}

Spatial interaction is a movement or transmission over space between at least two regions, which is resulting of a decision process involving different influences. Interaction as physical movement is for example migration movement, where nonphysical movement could be the transmission of messages or the exchange of knowledge.

The Spatial Interaction Model of two regions (Figure 2 ) is describing such spatial interaction with a relation, which is depending on different attributes. In the most general form, this model is given by the following equation:

$$
I_{i, j}=f\left(A_{i}, R_{j}, C_{i, j}\right)
$$




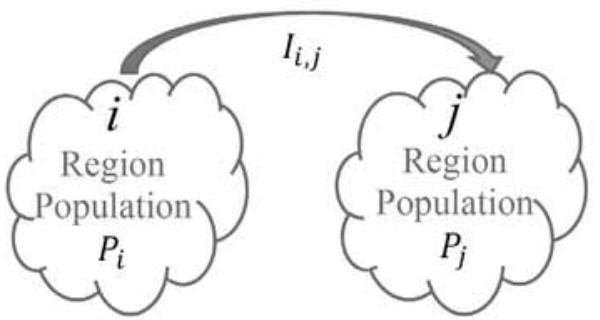

Figure 2. Population movement between regions.

The interaction factor $I_{i, j}$ ('Immigration'), responsible for movement from population in region $i$ to population in region $j$, is described by a function $f$ of attracting attributes $A_{i}$ in region $i$, of repelling attributes $R_{j}$ in region $j$ and separation attributes $C_{i, j}$ between region $i$ and region $j$ (Figure 2).

The interaction is depending on a decision process, which is happening based on certain conditions. For example, the decision of a commuter for a certain traffic route could be influenced by the costs of this route, the availability of public transport and the distance to work. These influencing factors are represented by attributes.

The shift of population is then given by $I_{i, j} \cdot P_{i}$, so that a time-dependent dynamics is introduced:

$$
\begin{aligned}
& P_{j, \text { next }}=P_{j, \text { prev }}+I_{i, j} \cdot P_{i, \text { prev }} \\
& P_{i, \text { next }}=P_{i, \text { prev }}-I_{i, j} \cdot P_{i, \text { prev }}
\end{aligned}
$$

\section{Social Gravity Models}

The widely used type of Spatial Interaction Models are the (Social) Gravity Models. Here the relation, which is describing the interaction, is based on Newton's law of gravity. This idea to draw analogies between physics and certain human behaviour has a long history. It came up in the year 1852 by Henry Charles Carey, who described the human migration behaviour as the 'tendency to gravitate the fellow man' [1]. In geography and demography, Gravity Models are used for a long time to analyse the flow of people, goods or capital. But often there is a lack of mathematical and theoretical foundation to understand the backgrounds ([2]).

This leads to examination of the relation between migration movement and the attraction of regions, as well as the distance between regions. Ernest Charles Young was the first who postulated the formal connection with the law of gravity [9]. Investigating the movement of farm population, he pointed out this coherence in the following formula:

$$
M=k \cdot \frac{F}{D^{2}}
$$

Here $M$ is the absolute migration, $F$ the intensity of attraction of a region, $D$ the distance to this region and $k$ a proportional constant. The dependency of attraction and the distance is based on Newton's law of gravity.

In the following years, this approach was used to describe human shopping behaviour and was refined by John Quincy Steward in the year 1941, who was developing the theory of demographic gravitation ([6]):

$$
I_{i, j}=G \cdot \frac{P_{i} \cdot P_{j}}{d_{i, j}^{2}}
$$

Here the influence of the fundamental physical law is obvious. The interaction $I_{i, j}$ between the population centres $i$ and $j$ is direct proportional to the product of the socalled population masses $P_{i}$ and $P_{j}$, which are describing the attributes in centres $i$ and $j$.

Furthermore the interaction is indirect proportional to the squared distance $d_{i, j}^{2}$ between the population centres $i$ and $j$. The constant $G$ is called the demographic gravity constant.

A few years later in 1950 Steward developed a formula, which involves the possibility of different impacts of the attributes to the interaction ([6]):

$$
I_{i, j}=G \cdot \frac{w_{i} P_{i} \cdot w_{j} P_{j}}{d_{i, j}^{2}}
$$

Here $w_{i}$ and $w_{j}$ are population weights. These weights are reflecting the heterogeneity of population masses and are treated as statistical parameters. With this parametric extension, the model description steps back from the direct analogy to Newton's law, but it increases the flexibility of the mode a lot.

After the introduced forms of gravity models, many different formalisations were used. Ashish Sen and Tony Smith were introducing a general class of gravity models ([6]).

The general class of gravity models has the following form

$$
I_{i, j}=A(i) \cdot B(j) \cdot F\left(d_{i, j}\right)
$$

where the interaction $I_{i, j}$ is resulting of the product of a weighted function $A(i)$ of attributes in $i$, a weighted function of the attributes $B(j)$ in $j$ and function of separation attributes $F\left(d_{i, j}\right)$ between $i$ and $j$. These weighted functions are vector-valued, so they can include a set of different attributes and heterogeneity.

SNE 27(4) - 12/2017 


\section{Migration Pathway Model}

Next aim is to develop a migration model with the pathway (network) of migration, which is fitting to the introduced class of gravity models. Therefore, the focus is on the migration behaviour between the regions of origin, the possible regions of destination and the transit regions.

The heterogeneity of the attraction or repulsion of these regions influencing the migration behaviour should be included. Furthermore, this attribute function should be treated as time dependent to observe structural changes in the migration behaviour over time. The model description should also have the flexibility to describe migration movement between a list of different included regions in different structure and through different routes.

\subsection{Graph model for pathway}

Directed graphs are a generic approach for describing connected regions and possible pathways of migration. Here, the graph of migration movement is defined as directed graph $G=(V, E)$, where the finite set of vertices

$$
V(G)=\left\{v_{1}, v_{2}, \ldots, v_{l}\right\}
$$

is describing the different regions of interest and the finite set of edges

$$
E(G)=\left\{e_{1}, e_{2}, \ldots, e_{k}\right\} \text { with } e_{r}=\left\langle v_{i}, v_{j}\right\rangle
$$

is describing the geographical possibility to migrate from one region $v_{i}$ to the other region $v_{j}$ (in the adjacency matrix of the graph denoted by a 1 ).

\subsection{Discrete model on pathway}

With this formalism, it is possible to describe a timedependent interaction $I_{i, j}(t)$ from region $v_{i}$ to region $v_{j}$ (Figure 3 ) with the following equation:

$$
I_{i, j}(t)=\frac{\sum_{k=1}^{n} a_{k} \cdot\left(A_{j}(t)\right)_{k} \cdot \sum_{k=1}^{m} r_{k} \cdot\left(R_{i}(t)\right)_{k}}{\sum_{k=1}^{n} c_{k} \cdot\left(C_{i, j}(t)\right)_{k} \cdot}
$$

If $M_{i}(t)$ and $M_{j}(t)$ denote the number of migrants in regions $v_{i}$ or $v_{j}$ resp., then the number of migrants $M_{i, j}(t)$ moving from region $v_{i}$ to region $v_{j}$ is given by

$$
M_{i, j}(t)=I_{i, j}(t) \cdot M_{i}(t)
$$

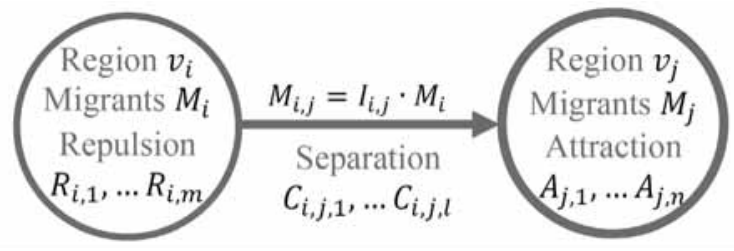

Figure 3. Migration movement between regions with attributes for attraction, repulsion, and separation.

Here the $n$ functions of attractive attributes $A_{j}$

$$
\left(A_{j}(t)\right)_{k=1}^{n}:[0, T] \rightarrow[0,1], a_{k} \in[0,1], \sum_{1}^{n} a_{k}=1
$$

in region $v_{j}$ are time-dependent, are normalized to 1 and are weighted by parameters $a_{k}$, which are the basis for following model identification. These properties also belong to the $m$ functions of repulsive attributes $R_{i}$ in region $v_{i}$

$$
\left(R_{i}(t)\right)_{k=1}^{m}:[0, T] \rightarrow[0,1], r_{k} \in[0,1], \sum_{1}^{m} r_{k}=1
$$

For the $l$ functions of separation attributes $C_{i, j}$

$$
\left(C_{i, j}(t)\right)_{k=1}^{l}:[0, T] \rightarrow[0, \infty), c_{k} \in[0,1], \sum_{1}^{l} c_{k}=1
$$

another value range is foreseen, in order to describe closed borders.

In general, time base for migration movement can be different - decades, years, quarters, months, and days. In any case, a synchronous time base results in a discrete dynamic model for migration from the region $v_{i}$ to region $v_{j}$, using the actual migration equation with the interaction factor $M_{i, j}(t)=I_{i, j}(t) \cdot M_{i}(t)$ :

$$
\begin{aligned}
& M_{j}(t+1)=M_{j}(t)+I_{i, j}(t) \cdot M_{i}(t) \\
& M_{i}(t+1)=M_{i}(t)-I_{i, j}(t) \cdot M_{i}(t)
\end{aligned}
$$

Clearly, any region may have more than one neighbour with immigration and emigration, so that in the above equations, more bilance terms will appear, but structurally only neighbouring regions are taken into account:

$M_{j}(t+1)=M_{j}(t)+M_{i, j}(t)+\sum M_{r, j}(t)-\sum M_{j, s}(t)$
$M_{i}(t+1)=M_{j}(t)-M_{i, j}(t)-\sum M_{u, i}(t)+\sum M_{i, v}(t)$ In above equations, the sums spread over neighbouring emigration regions and immigration regions, except region $v_{i}$ and region $v_{j}$, which are directly balanced in the equations. 


\subsection{Discrete model with transit regions}

The consideration of only neighbouring regions in the up to now developed model may be sufficient for regions, which all are potential immigration regions. Especially in the case of refugee waves, some regions are only transit regions, which have high repelling attributes and which must have attractive attributes; but these attractive attributes are not a property of the regions itself, they are given by the attractive properties of the immigration neighbours of the transit region.

This fact requires a model extension for the calculation of attractive attributes of a transit region $v_{j}-$ using the attractive attributes of the neighbouring immigration regions $v_{j_{1}}, v_{j_{2}}, \ldots v_{j_{u}}$ given by

$$
A_{j_{r}}^{*}=\sum_{k=1}^{n} a_{k} \cdot\left(A_{j_{r}}(t)\right)_{k}
$$

Instead of the generic summed up attractive attributes $A_{j}^{*}$, the maximum of summed up attractive attributes of neighbouring immigration regions is used:

$$
A_{j}^{+}(t)=\max _{u=j_{1}, \ldots, j_{u}} A_{u}^{*}(t)
$$

Also the separation attributes $C_{j, j_{r}}^{*}$ from transit region $v_{j}$ to the neighbouring immigration regions $v_{j_{1}}, v_{j_{2}}, \ldots v_{j_{u}}$ must be taken into account - one appropriate solution is to make use of separated maximum calculation:

$$
A_{j}^{+}(t)=\max _{u=j_{1}, \ldots, j_{u}}\left(A_{u}^{*}(t) \cdot \max _{v=j_{1}, \ldots, j_{u}} C_{j, v}^{*}(t)\right)
$$

Additionally also the repulsion attributes $R_{j}^{*}(t)$ are increased to $R_{j}^{*}(t)$ depending on a certain transit category. Figure 4 sketches these extensions for transit.

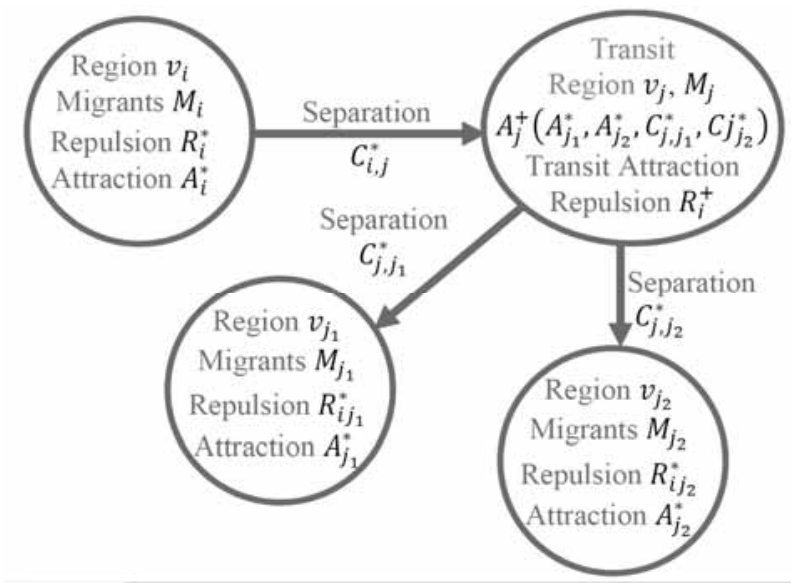

Figure 4. Migration movement through a transit region with dependent attraction attributes.

\subsection{Relation to social behaviour modelling}

Since the presented discrete model is simulating social behaviour, it must be mentioned, that the social aspects and reasons behind the migration are a result of a complex composition of various influences. Finding appropriate descriptions for social behaviour is a great challenge, and the available different approaches are suited to cover the different aspects, but not the overall behaviour. As consequence, the characterization of the presented migration model is important in terms of understanding the qualities and limits of this approach.

Empirical studies showed that gravity models are most successful in describing macroscopic patterns of spatial interaction. They are more reliable in picturing the behaviour of populations rather than individuals ([6]). One reason is that individual decisions are often influenced by many different partly unknown factors, which refer to specific individuals. Here, agent-based modelling may have better merits, but less features for quantitative identification. On the other hand, the focus on population groups gives the quality of a sufficient description of the behaviour of interest, with just a little required information.

It is also important to discuss the aspect of time. The dynamics of the introduced migration model are only observed at discrete time steps, staying constant over the next period of time. Structural changes, for example a change of the attribute function from one time step to the next one, are 'treated' as event. From this viewpoint, the model shows quasi-static behaviour, depending on the chosen time base.

The model is by mathematical definition a deterministic model, but most of the parameters are rates - averages of probabilistic distributions. From this viewpoint, the model is averaging probabilistic behaviour (details on these considerations see [8]).

\section{Refugee Crisis Model}

The presented discrete model with transit regions allows modelling the pathway of refugees during the so-called Refugee Crisis 2015. Begin of September 2015 many people fled from war actions in Syria, and at the same time some of the refugee camps in Lebanon were shut down due to financial reasons - resulting in a refugee wave towards Western Europe. Figure 1 shows snapshots of data animation of refugees' pathway in the Refugee Crisis 2015 sketching the principle structure of the pathway. 


\subsection{Model graph structure}

For model implementation, the pathway is defined by a graph with nineteen vertices - the evident regions along the pathway. Origin is vertex $v_{1}$, region Syria, and final destinations are regions in Western Europe. It is not necessary - and because lack of data not possible - to investigate all countries on the pathway as self-consistent vertex, some countries are considered as common region along the pathway from Syria to Western Europe.

Table 1 lists the regions - the vertices implemented in the model, and Figure 5 shows the graph with the possibilities for migration.

\begin{tabular}{|c|c|}
\hline Vertices & Regions \\
\hline$v_{1}$ & Syria \\
\hline$v_{2}$ & Iraq \\
\hline$v_{3}$ & Jordan \\
\hline$v_{4}$ & Egypt \\
\hline$v_{5}$ & Lebanon \\
\hline$v 6$ & Turkey \\
\hline$v_{7}$ & Greece \\
\hline$v_{8}$ & Former Republic of Macedonia \\
\hline$v_{9}$ & Serbia \\
\hline$v_{10}$ & Hungary \\
\hline$v_{11}$ & Croatia \\
\hline$v_{12}$ & Slovenia \\
\hline$v_{13}$ & $\begin{array}{l}\text { Slovakia, Czech Republic, } \\
\text { Rumania, Bulgaria, Poland, } \\
\text { Lithuania, Estonia, Latvia }\end{array}$ \\
\hline$v_{14}$ & Austria \\
\hline$v_{15}$ & Germany \\
\hline$v_{16}$ & $\begin{array}{l}\text { United Kingdom, The Netherlands, } \\
\text { Belgium, France }\end{array}$ \\
\hline$v_{17}$ & Norway, Finland, Sweden, Denmark \\
\hline$v_{18}$ & Italy, Spain, Portugal \\
\hline$v_{19}$ & $\begin{array}{l}\text { Albania, Bosnia and Herzegovina, } \\
\text { Montenegro }\end{array}$ \\
\hline
\end{tabular}

Table 1. Regions - graph vertices - under consideration for Refugee Crisis model.

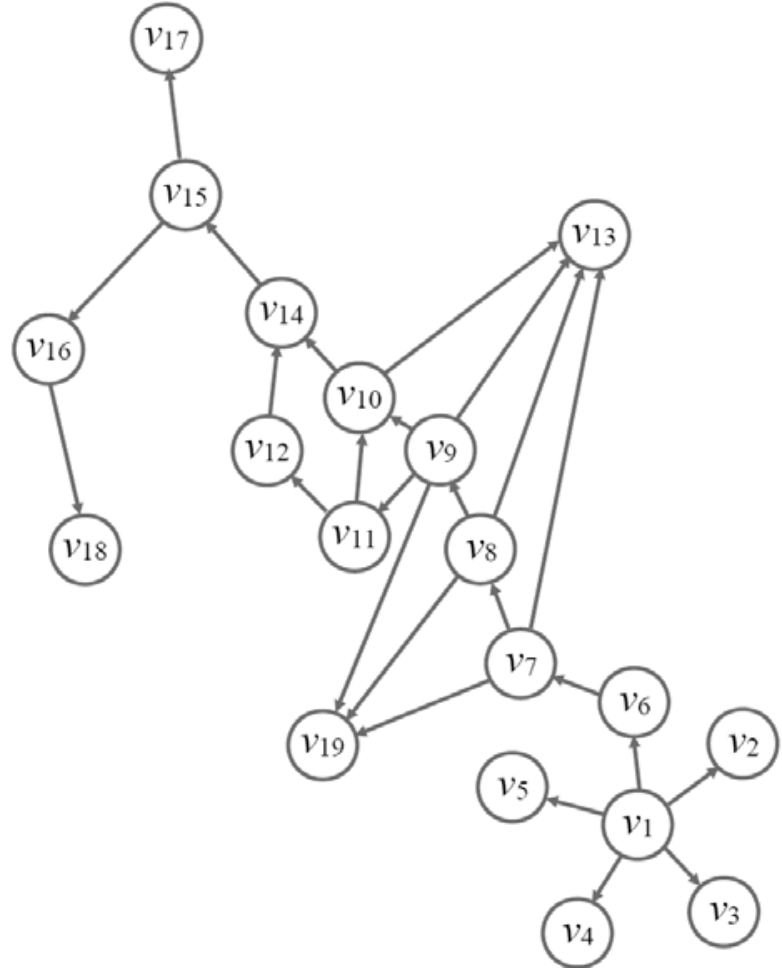

Figure 5. Migration graph model for Refugee Crisis 2015 from Syria into and within Europe in September/October 2015.

\subsection{Attribute functions}

The model focusses on the migration movement and the different migration routes and it is not modelling any reasons for emigration. This implies a distinction of countries of origin and potential destination countries. Observing the interactions from Syria to the neighbouring countries, the repulsive attributes of the country of origin are not taken into consideration. Here the attractive attributes of the neighbouring countries are determining the intensity of interaction.

Necessary is also a distinction between potential destination countries and transit countries. The attractive attributes of a transit country have less influence on the interaction; they are overlaid by the attraction attributes of the next destination regions. In the model, a transit region (country) is defined by a region, the repulsion attribute of which exceeds a certain limit. In this case, the attractive attributes of the region are replaced by an appropriate maximum of the attractive function of the countries, which are accessible from the transit region (formulas see Section 3.3). 
The choice of attraction attributes and repulsion attributes follows investigations and negotiations published in a study of the Australian economic scientist Timothy Hatton ([4]), and investigations by Neumayer on asylum destination choice ([5]). Hatton examined the correlation of specific attributes of developed countries and the number of asylum applications. As essential, he observed a big effect of access policy, like the border security, and of processing policy, like the humanitarian situation. Interestingly, the study shows only a small positive impact of welfare policy on the number of asylum applications ([4]). Table 2 and Table 3 list the seven attraction attributes and the five repulsion attributes used in this model implementation.

\begin{tabular}{cl}
\hline Function & Attraction Attributes \\
\hline $\boldsymbol{A}_{1, j}$ & Gross Domestic Product (GDP) \\
\hline $\boldsymbol{A}_{2, j}$ & Fragile State Index (FSI) \\
\hline $\boldsymbol{A}_{3, j}$ & Migrants in the country \\
\hline $\boldsymbol{A}_{4, j}$ & $\begin{array}{l}\text { Attractive attributes of accessible } \\
\text { countries }\end{array}$ \\
\hline $\boldsymbol{A}_{5, j}$ & Not exceeded capacity \\
\hline $\boldsymbol{A}_{6, j}$ & Asylum recognition rate in country \\
\hline $\boldsymbol{A}_{7, j}$ & Asylum recognition quote in Europe \\
\hline
\end{tabular}

Table 2. Seven attraction attributes for countries (regions) along pathway, $\mathrm{j}=1, . .19$.

\begin{tabular}{cl}
\hline Function & Repulsion Attributes \\
\hline $\boldsymbol{R}_{\mathbf{1}, j}$ & Gross Domestic Product (GDP) \\
\hline $\boldsymbol{R}_{\mathbf{2}, j}$ & Fragile State Index (FSI) \\
\hline $\boldsymbol{R}_{\mathbf{3}, j}$ & Exceeded capacity \\
\hline $\boldsymbol{R}_{\mathbf{4}, j}$ & Asylum recognition rate in country \\
\hline $\boldsymbol{R}_{\mathbf{5}, j}$ & Asylum recognition quote in Europe \\
\hline
\end{tabular}

Table 3. Five repulsion attributes for countries (regions) along pathway, $\mathrm{j}=1, . .19$.

The access policy and access control of a country is described by appropriate separation functions $C_{i, j}(t)$. In case of (partly) closed borders or closed borders enforced by border security arrangements, the separation functions reduce interaction immediately.
In general, time base for migration movement can be different - decades, years, quarters, months, and days. The investigated migration waves last one to three months, and data are available for the Refugee Crisis 2015 on a daily base, so a time base of days is appropriate for the model to be implemented. Clearly, the time base scales the interaction function $I_{i, j}$, and indirectly all attribute functions.

The unknown parameters in the model are the weighting parameters $a_{r}, r=1, \ldots, 7$ and $r_{s}, s=1, \ldots, 5$ in the formulas for the interaction function $I_{i, j}$. The parameters may differ for some countries, especially for the country of origin; some can be estimated, some must be identified using the data reported from UNHCR.

Figure 6 and Figure 7 show examples for the weighting parameters of attractive and repulsive attributes in classical destination countries. Of course, the attractive attributes of the county of origin must follow an exclusive non-attractive weighting - Figure 8.

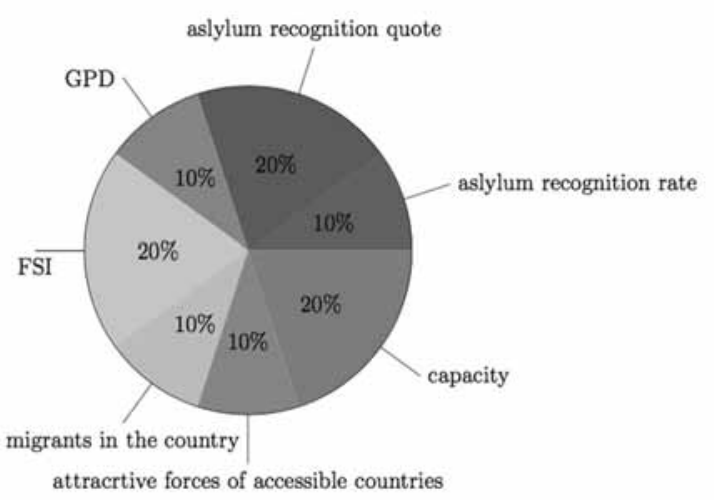

Figure 6. Weighting parameters for attractive attributes potential destination country.

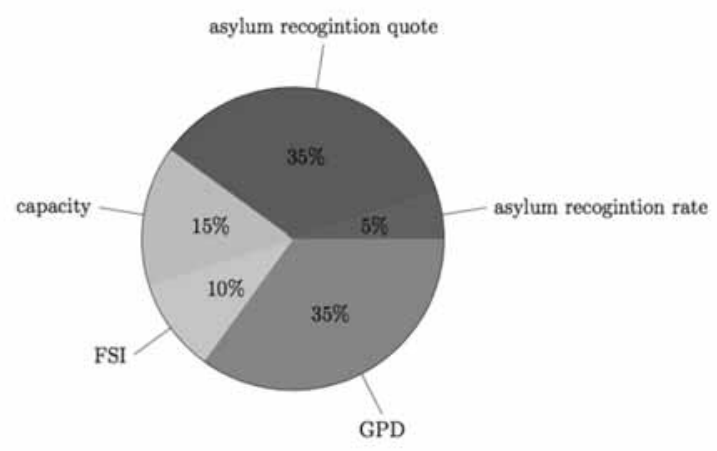

Figure 7. Weighting parameters for repulsive attributes potential destination country. 


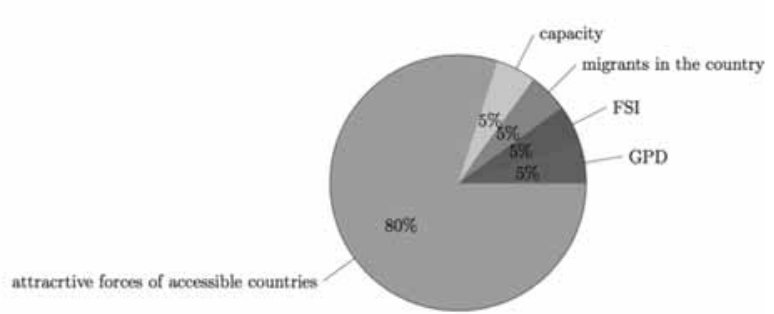

Figure 8. Weighting parameters for attractive attributes country of origin

\subsection{Software implementation}

The model description with the sum formulas and with the multiple indices looks difficult to implement. However, the graph structure with vector of vertices and adjacency matrix, and the possibility to formalize the attributes as vectors allows a compact model implementation in MATLAB language - code snippet in Figure 9.

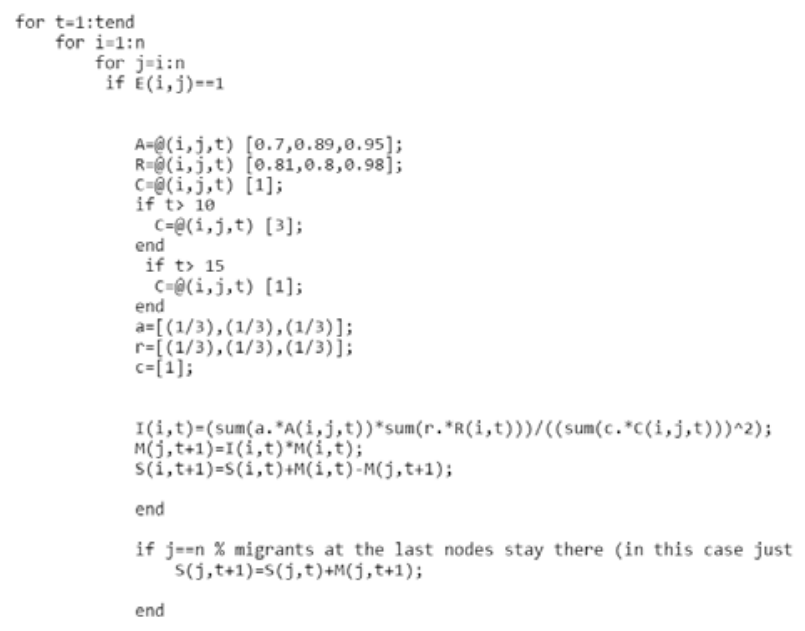

Figure 9. Model implementation in MATLAB - vector and matrix structures within main loop.

\subsection{Simulation results}

The implemented model allows a simulation of the pathway of refugees during the Refugee Crisis 2015, in the period from September 1, 2015 to October 31, 2015. A big challenge was the proper choice of the parameters the weighting parameters for the attributes. Some of them can be determined by available data, some must be identified in comparing UNHCR data from daily migrant movement with results from the simulation.

Most of the attraction attributes and repulsion attributes can be seen constant during these 61 days. At the first glance, the migration movement seems to be linearly growing over time, as shown with the number of refugees in the regions over time (Figure 10).

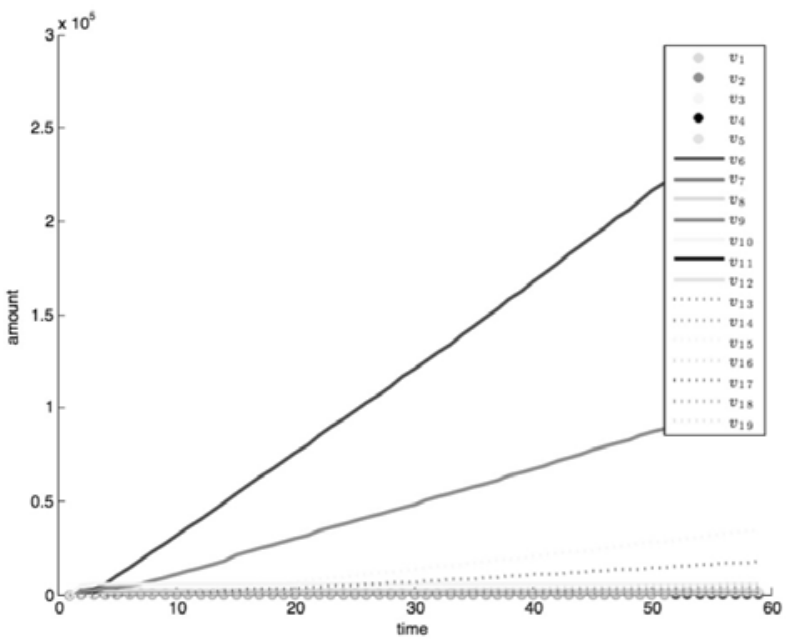

Figure 10. Result from simulation of Refugee Crisis 2015: number of refugees passing the 19 regions - almost linear growing behaviour.

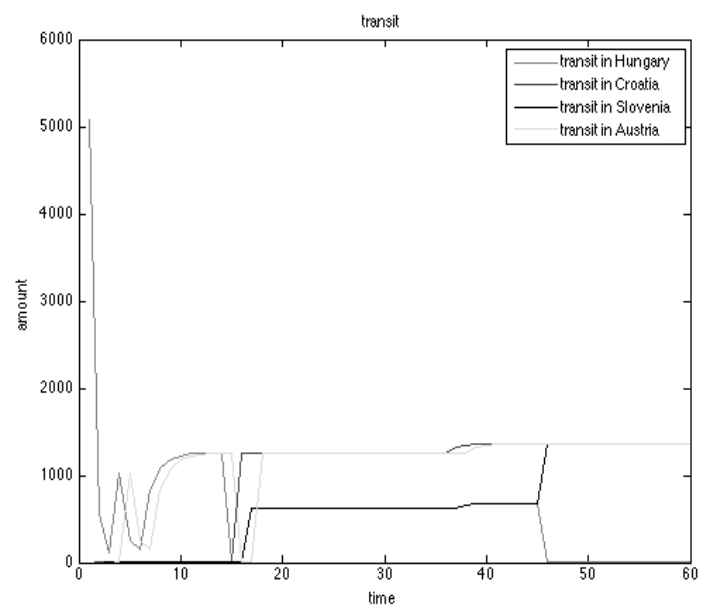

Figure 11. Result from simulation of Refugee Crisis 2015: number of refugees passing Hungary, Croatia, and Slovenia under impact of closing of borders on September 15 and 16, 2015.

The separation attributes clearly are not constant, they reflect the closing of the border from Hungary to Serbia on September 15, 2015, and the closing of the border from Hungary to Croatia on October 16, 2015, and subsequent opening of borders. Figure 11 impressively demonstrates the effect of the closing of borders on the amount of refugees trying to pass these borders. Clearly, under these circumstances the linear growth is replaced by constant values (and zero values) for migration.

The quality of the results can be checked by comparison with UNHCR data, at best with the summed-up number of asylum applications in each region. 


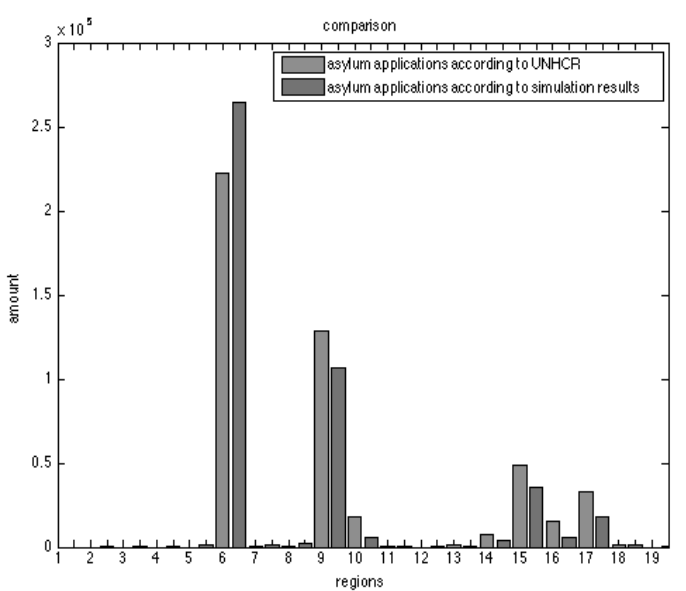

Figure 12. Result from simulation of Refugee Crisis 2015: summed up number of asylum applications in the 19 regions (right column, blue) compared with UNHCR data (left column, green) at the end of the refugee wave.

Simulation results in Figure 12 show the results of the migration movements as summed up number of asylum applications (during the full period) in the nineteen regions and compares these results with data recorded by UNHCR.

Some of the numbers in Figure 12 seem to be astonishing, as many people expect the highest number of asylum applications in Western Europe. However, the highest number of applications were put in vertices $v_{6}$ and $v_{9}$ - Turkey and Serbia. Significantly lower are the applications in the vertices $v_{15}, v_{17}, v_{16}$ and $v_{14}$-Germany, Scandinavia, France/UK, and Austria.

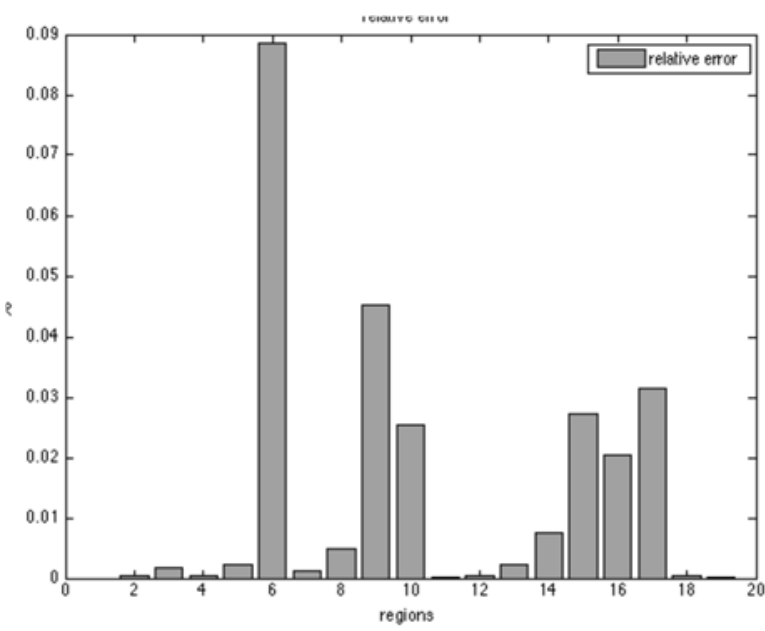

Figure 13. Result from simulation of Refugee Crisis 2015: relative error for summed up number of asylum applications in the 19 regions (simulation results data vs. UNHCR data).
The comparison of data from simulation and UNHCR data for the summed-up number of asylum applications allows calculating a relative error for the simulation result data, displayed in Figure 13. For most regions, the relative error is below $5 \%$, only for vertex $v_{6}$ - Turkey - it goes up to $9 \%$. Such relative errors are not satisfying for simulations of technical systems, but for socio-economic systems these errors are acceptable and lower than expected.

\subsection{Animation of simulation results}

Time plots can present results of the simulation, e.g. the number of migrants, as with Figure 10 and Figure 11, or bar charts with summed-up number of asylum applications. However, it is challenging to display also the pathways. Here, an animation of this specific refugee wave was implemented by $d w h$ Simulation Service for ORF 1 Online (Austrian Broadcast Company, Web Science Program Online).

The web-based animation with a sequence of 61 maps of Europe show for each of the 61 days

- the pathway of migrants by arrows from regions to region (the thickness of the arrow indicates qualitatively the up-to-data summed-up number migrants,

- the summed-up number of transit migrants and migrants applying for asylum in each region,

- and border actions by prohibitory signs at restricted or closed borders.

Figure 1 already presented two snapshots of this animation, but data driven. Now this animation can be driven by simulation data: Figure 14 and Figure 15 show snapshots, the one from begin of the refugee wave, and the other from end of wave, after the closing of borders.

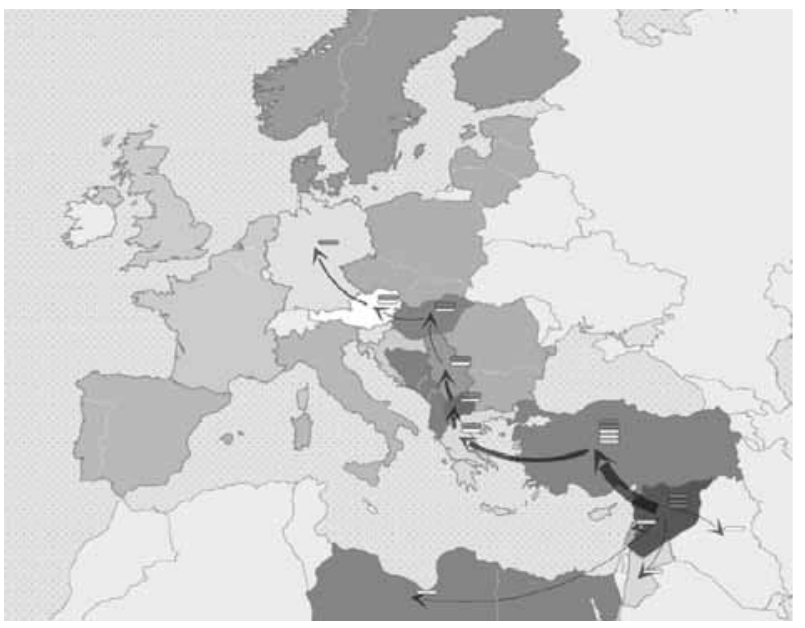

Figure 14. Refugee Crisis 2015: Simulation data animation, snapshot from begin of refugee wave.

SNE 27(4) - 12/2017 


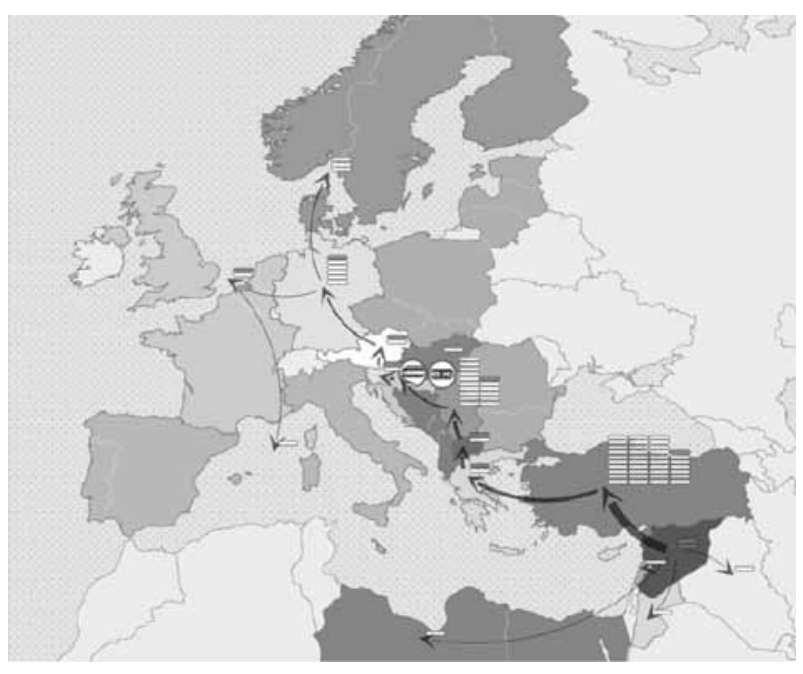

Figure 15. Refugee Crisis 2015: Simulation data animation, snapshot from end of refugee wave, with border closing at Balkan route.

\section{Forecast Model}

The Refugee Crisis Model allows an a-posteriori simulation of the Refugee Crisis 2015: the model was developed after the refugee wave, and the simulation results could be verified by data reported during the refugee wave. As the value of modelling and simulation is mainly forecast of dynamic behaviour, the question arises, whether the model can simulate a pathway of migration in advance a need for a Forecast Model.

In spring 2016, the so-called Balkan Route was closed by border control and by the so-called Turkey Deal. Nevertheless, for June 2016 again a refugee wave was expected, using the Mediterranean route. The challenge was to forecast parts of this wave by simulation, using a modification and extension of the Refugee Crisis Model.

The main changes for the Forecast Model are

- change of regions

- change of pathways, and

- change of some attributes.

Main task was the implementation of the Mediterranean route. An easy and generic solution is to continue the already existing pathway to Africa and connect it with the northern Mediterranean coasts. As consequence, vertex $v_{4}$ - Egypt - changes to vertex $v_{4}$ - North Africa/Coast, and the sea route is defined by a new pathway from vertex $v_{4}-$ North Africa/Coast to vertex $v_{18}$ - Italy, Spain, Portugal.

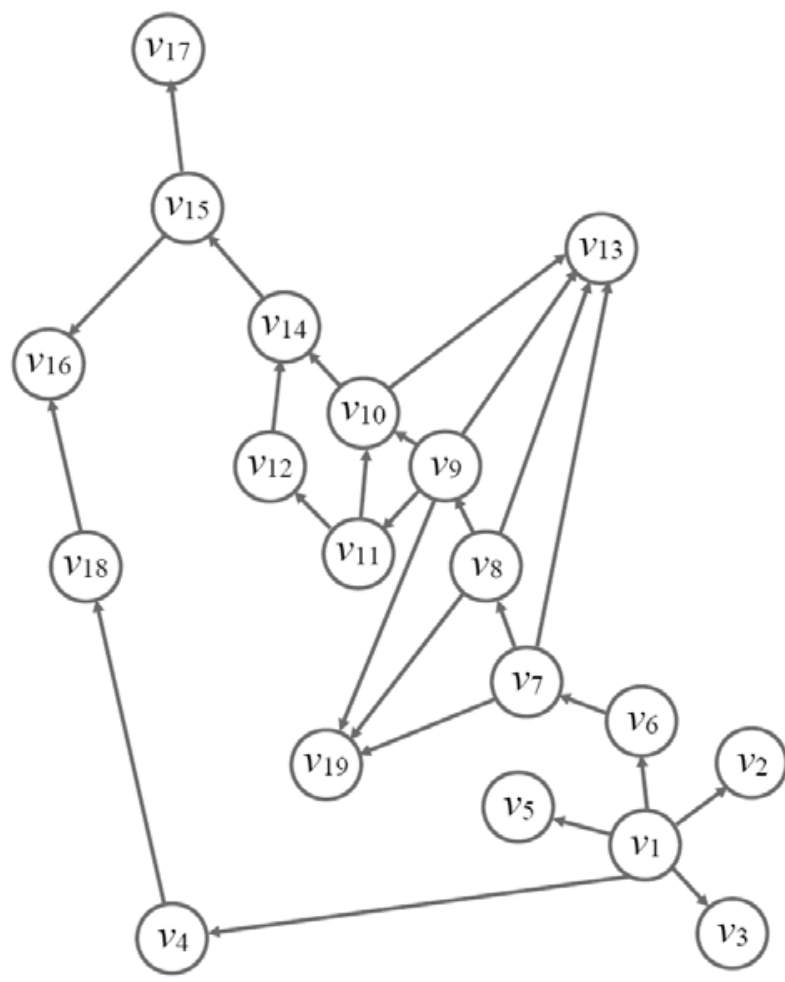

Figure 16. Migration graph model for Forecast Model with Mediterranean Sea route.

Figure 16 shows the new migration graph, with pathway along the Mediterranean Sea Route. It is not necessary to take into account a pathway from vertex $v_{4}$ - North Africa/Coast to vertex $v_{7}$ - Greece-because of the Turkey Deal, (as with the pathway to Balkan coast, because of the closed Balkan Route).

The Forecast Model can re-use most of the parameters and values of the attributes, except separation attributes, and some attraction attributes. The separation attributes $C_{i, j}$ of regions at Balkan model a closure of the borders for migrants, and separation attributes in Middle Europe (vertices $v_{15}, v_{17}$ - Germany, Scandinavia,) are increased (stronger border control for migrants). Furthermore it is assumed, that vertex $v_{16}-$ Western Europe has exceeded the asylum capacity (lower attraction attributes).

The model is parametrized to forecast by simulation the pathway of migrants and the number of asylum application for June 2016, with the same time base as the previous model. The simulation results calculated before the June 2016 migrant wave can now be compared with available data collected during and at the end of June 2016 migrant wave - displayed in barcharts for the asylum applications in Figure 17. 


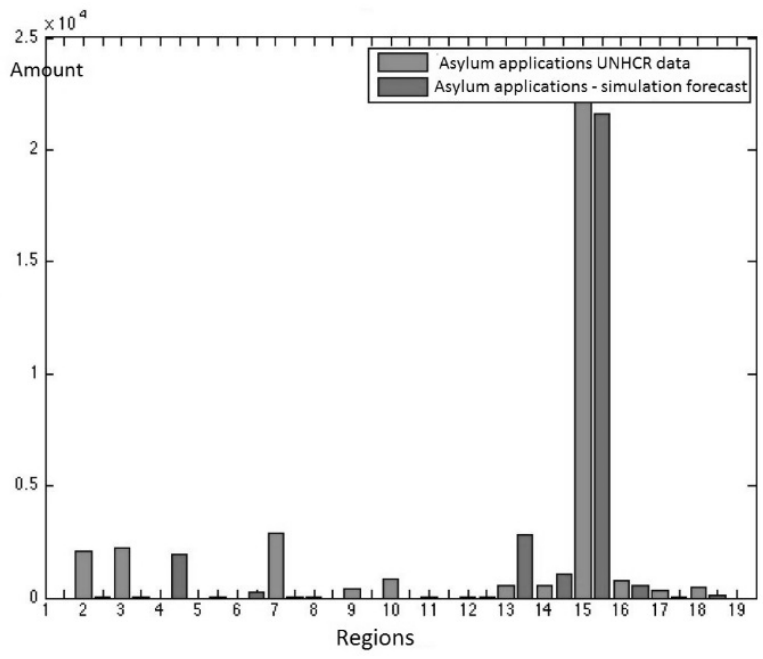

Figure 17. Forecast Model - June 2016 Migrant Wave summed up number of asylum application in the 19 regions (right column, blue) compared with compared with UNHCR data (left column, green) at the end of the refugee wave.

The simulation results are partly sufficient. Migrants put most asylum applications in vertex $v_{15}$ - Germany, here simulation results coincide well with a-posteriori data (8\% relative error). Satisfying are also results for $v_{16}-$ Western Europe (15\% relative error) and $v_{14}$ - Austria (26\% relative error). The significant higher relative errors for Balkan and Mediterranean regions are partly based on the fact, that data were not available, so that average data from spring 2016 were used. An exception is vertex $v_{7}$-Greece: the high number of real applications seems to include applications from migrants, who arrived earlier, and who had to stay because of the closure of the Balkan Route.

The relative errors of parts of the results of the Forecast Model are high. Such relative errors are not satisfying for simulations of technical systems, but for socioeconomic systems these errors are acceptable, especially they still allow a qualitative analysis of the dynamic behaviour.

Indeed the power of the Forecast Model is the possibility of a qualitative forecast of the migration pathway, especially when displayed in ascertainable manner for time domain, spatial domain, and flow domain, as with the web-based animation of maps of Europe with dynamic migration. Figure 18, Figure 19, and Figure 20 show three snapshots from this animation, the first at begin of June with closed borders, the second midst of June with already frequently used Mediterranean Sea route, and the third end of June, with final data.

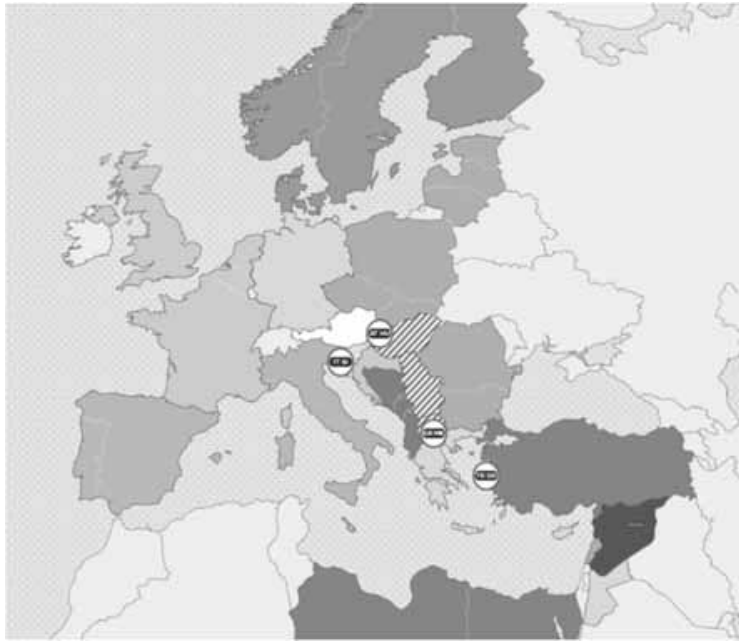

Figure 18. Forecast Model - Refugee Wave June 2016: simulation data animation, snapshot at begin.

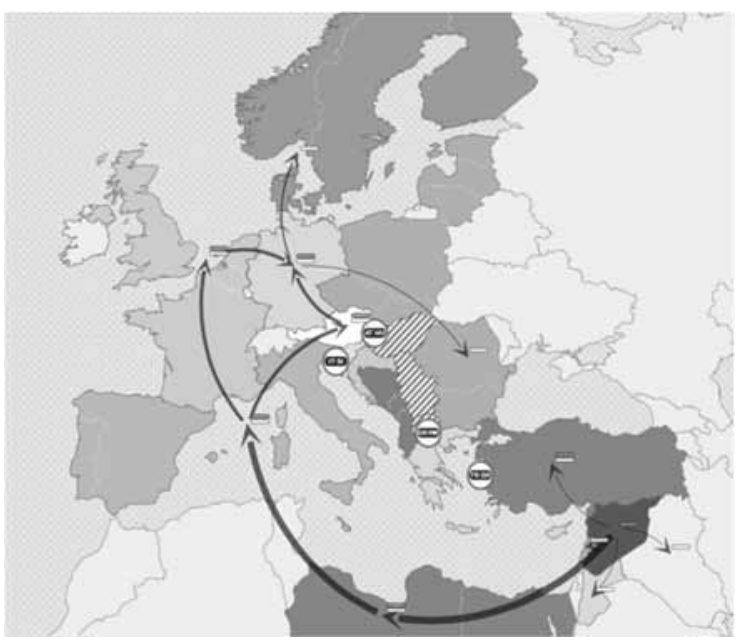

Figure 19. As Fig. 18, snapshot midst of June, frequently used Mediterranean Sea Route.

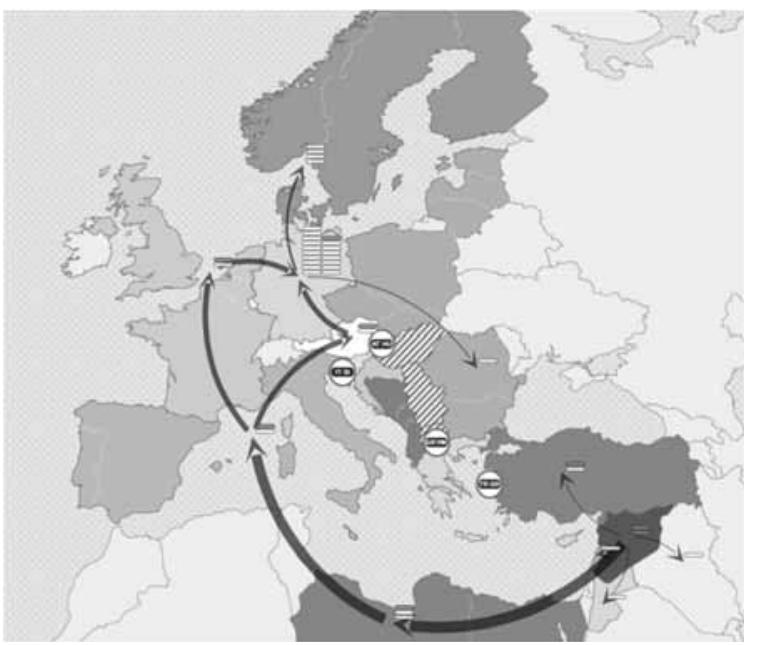

Figure 20. As Fig. 18, snapshot end of June, frequently used Mediterranean Sea Route and distribution of asylum applications. 


\section{Scenario Model}

Last step in the model development was the parametrization of the Forecast Model with average values for attraction attributes and for repulsion attributes, on basis of the structure with 19 regions. Additionally, this model provides some special separation attributes in order to study certain separation control, among them the closing of the Brenner border, the closing of German borders, etc.

This model allows analyzing qualitatively these scenarios by means of the barcharts for asylum application, and by means of the animated maps of Europe with migration pathways. Figure 21 shows one of these scenarios: Balkan Route closed, German borders closed - the result is a very strong pathway along the Mediterranean Sea Route - with all side effects.

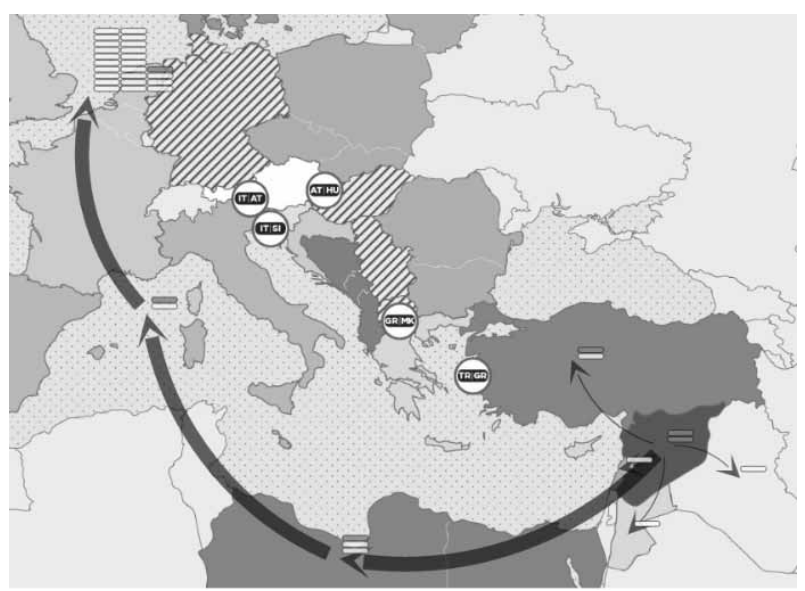

Figure 21. Scenario Model - Refugee Wave with extensive use of Mediterranean Sea Route.

\section{Conclusion}

'Models are in any case a simplification of reality, but they should help in better understanding of complex dynamics as migration movement, and the intention of this model is to show strategies to improve the situation of migrant population and resident populations under appropriate prerequisites.'

The above statement expresses the hope of the authors that the simulation really helps in understanding better pathways of migrants and refugees, and to think of the human beings involved. Clearly, the attributes in the regions control the flow of migrants. Still politicians think only on control by separation attributes (let's close the borders!), instead of thinking on an evenly distribution of attraction attributes in all regions, or on decrease of repulsion attributes (welfare, no wars, ...).
Many people think that figures with pathways of migrants along the Mediterranean Sea Route are a novelty in Europe (e.g. Figure 29 as reality, or Figure 21 as scenario). The main author found a very similar picture with pathways of migrants along the Mediterranean Sea Route (see below) in an article by J. Fischer about the migration of the sea people at the end of Bronze Age ([3]) - let's also learn from history!

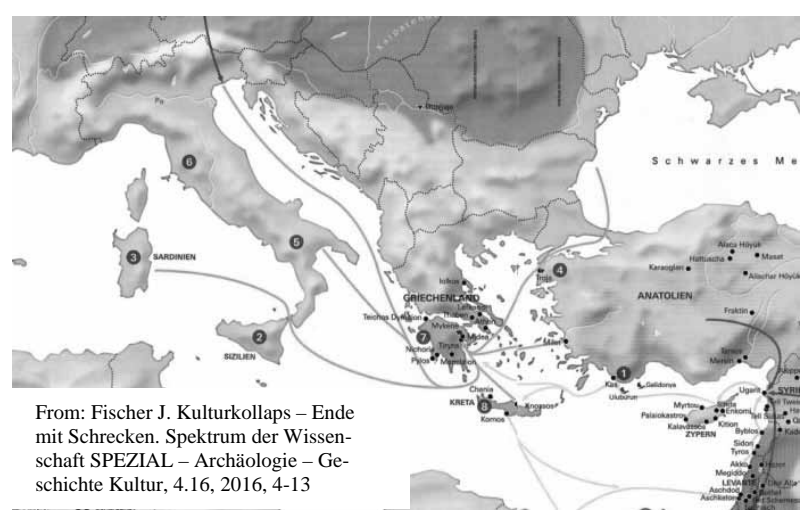

\section{References}

[1] Carey H C. Principles of Social Sciences. Philadelphia, Pennsylvania 1858.

[2] Dennett A. Estimating flows between geographical locations: get me started in spatial interaction modelling. Working Paper Series, Paper 181-March 12, UCL, Centre for Advanced Spatial Analysis, 2012.

[3] Fischer J. Kulturkollaps - Ende mit Schrecken. Spektrum der Wissenschaft SPEZIAL - Archäologie Geschichte Kultur, 4.16, 2016, 4-13.

[4] Hatton T, Maloney. Applications for Asylum in the Developed World: Modelling Asylum Claims by Origin and Destination. ANU Working Papers in Economics and Econometrics, 625, Australian National Univ. 2015.

[5] Eric Neumayer. Asylum Destination Choice: What makes some west European countries more attractive than others?, European Union Politics, Volume 5 (2), 155-180, SAGE Publications, 2004, London.

[6] Sen A, Smith T. Gravity Models of Spatial Interaction Behaviour. Springer, 1995.

[7] Vobruba T. Modellbildung, Analyse und Simulation eines räumlichen Interaktionsmodells. Master Thesis TU Wien, 2016, p.115.

[8] Vobruba T, Körner A, Breitenecker F. Modelling, Analysis and Simulation of a Spatial Interaction Model. Proc. 17th IFAC TECIS Conf., P. Kopacek, E. Hajrizi (eds); IFAC-PapersOnLine Volume 49, Issue 29, 221-225. doi 10.1016/j.ifacol.2016.11.054

[9] Young E C. The movement of farm population. Cornell Agricultural Experiment Station, New York, 1924. 


\title{
Planning Future Health:
}

\section{Developing Big Data and System Modelling Pipelines for Health System Research}

\author{
Niki Popper ${ }^{1 *}$, Florian Endel ${ }^{2}$, Rudolf Mayer ${ }^{3}$, M artin Bicher $^{2}$, Barbara Glock ${ }^{1}$ \\ ${ }^{1}$ COCOS - Centre for Computational Complex Systems, TU Wien, Wiedner Haupstrasse 8-10, \\ 1040 Vienna, Austria; *nikolas.popper@ tuwien.ac.at \\ ${ }^{2}$ DEXHELPP, Neustiftgasse 57-59, 1070 Vienna, Austria; ${ }^{3}$ SBA Research, Favoritenstrasse 16, 1040 Vienna, Austria
}

SNE 27(4), 2017, 203 - 208, DOI: 10.11128/sne.27.tn.10396 Received: December 2, 2017; Revised: December 15, 2017; Accepted: December 22, 2017

SNE - Simulation Notes Europe, ARGESIM Publisher Vienna, ISSN Print 2305-9974, Online 2306-0271, www.sne-journal.org

Abstract. Using Modelling and Simulation for Planning and Evaluation of Health Systems needs the combination of state of the art modelling and simulation tools, which can be used in a modular way. In addition, big data sets are used to describe the status-quo of health systems. Together possibilities for prediction and strategic planning increase, while challenges for quality assessment and reproducible processes generate the need for new developed structures in research and implementation. The article gives an outline of approaches, developed within the Austrian DEXHELPP project and points out strategies for coping with these challenges.

\section{Introduction}

Based on the concepts of equations, networks, algorithms and causal understanding of the world, modelling and simulation has reached a high level of describing systems and processes, like complex technical systems, ecological systems or production and logistic processes. Challenges are developing, combining, transferring and communicating such approaches and solutions.

On the other hand, big data has come to an eminent importance based on sensors and computational efforts in measuring our world. Today, many technologies for the construction, monitoring, and evaluation exist. A huge amount of activities can be seen in research, founding, policy and media.

Still, interfaces and methods for linking these technologies are to be intensified. Especially complex socio- technical systems intertwining technologies and humans, aiming to serve the goals of citizens on different levels, ranging from the personal goals of a citizen regarding, e.g., treatment and prescription management to the management of Health Care by policy and decision makers. One prerequisite to achieve this is the analysis of actual data and forecasting of the future behaviour with simulation methods.

\section{Future Health Systems}

National health systems invest annually more billions of Euros as the demand for health services increases (because of demographic change), but resources are limited. In addition, complexity of processes increases: From diagnosis to therapy - Interventions are complex hybrid processes including e-health, decentralised services and personalised medicine.

Measurement of efficiency and effectiveness becomes more and more complex but is an urgent need. Development of new methods, models and technologies is needed to support analysing, planning and controlling. Quantity and quality of available data strongly increases and therefore facilitates the description and analysis of all areas in complex systems like health care. Based on data for healthy expenditures the evaluation for health care systems has a market of 75 to 120 Billion Euro only in the European Union.

To provide state of the art analysis for Health Technology Assessment (HTA), Comparative Effectiveness Research (CER) and Evidence Based Medicine (EBM) processes combining health system domain knowledge, knowledge of professional data processes and - finally mathematical modelling \& simulation will be vital to transform Big Data into Deep Data: Evidence based and reproducible knowledge (See $[1,2])$. 
Bringing together these technologies is an enormous challenge. Data Based Demographic models must be combined with models for the spread of diseases. Time dependent treatment paths must be parametrized with data sets from clinical routine joined with large scale health system data. For system simulation an important aspect is the possibility to implement changes inside the system, like interventions within the computer model, and to analyse their effects.

On basis of experiences of the Austrian DEXHELPP COMET project for Decision Support in Health Policy and Planning, where an innovative research infrastructure was developed to enable researchers and other stakeholders to share data and methods for research and decision making, some important points to handle these complex processes in future will be described.

\section{Big Data and Complexity}

Next generation tools are needed to make development, construction monitoring, and analysis of such systems easier, faster, more reliable and - one of the most important things - comprehensible for decision makers and other stakeholders. To achieve these goals, methods in the following areas must be developed:

- Data: Integration, storage, management and analysis of very large amounts of data, unstructured data, secure and reproducible data management from sensors, IoT and various data sources like dynamic databases or non-structured information sources, collecting data, interfaces and analysis methods from statistics, machine learning, and visual analysis.

- Models: Formal, scalable modelling of various systems, heterogeneous modelling of subsystems and the integration of these subsystems, development of modelling methods for computational complex systems, multi method modelling, including coupling and comparison based on data, system knowledge and applications demands.

- Computation: Combining data and models to simulation tools for complex systems, developing innovative methods in numerical mathematics, cosimulation, hybrid simulation and reproducible simulation.

- Interfaces: Interfaces and visualization of simulation results, decision support systems and future development of Human-Computer Interaction (HCI).

\section{Data Infrastructures}

Data required in scientific investigations in the health domain is in many cases difficult to share, as it contains personalized information, e.g. on patients and service providers in the health sector. However, detailed and high-grade data is required to compute high-quality mathematical and statistical models and visualizations of interesting facts.

To facilitate sharing of data, infrastructure, software tools and well-defined processes must be developed to enable researchers and customers to share their data in a secure and transparent way. DEXHELPP developed a prototype for support of Austrian health system research, sharing information between research community and the health system stakeholders.

\subsection{Infrastructure for research}

Computational sciences such as e-Health depend heavily on the detail and quality of the data that is utilised to develop mathematical and statistical models. However, especially with data in the health sector that often comprises sensitive data on people, protection of the privacy of these individuals is often impeding easy exchange of data between data holders and consumers.

DEXHELPP tackles this problem by having created a secure and controlled environment (see below Figure 1) where data holders can deposit their data, and data consumers can perform their analysis and experiments within that environment, without the need to transfer the data outside of the system.

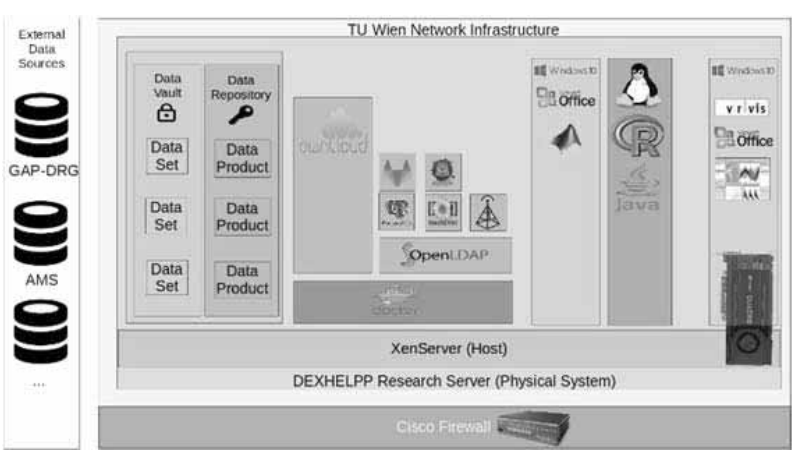

Figure 1: Overview of the architecture of the DEXHELPP Research Server infrastructure.

Data providers can specify fine-grained access rights to individuals or groups of researchers, to complete data sets or just specific subsets thereof, e.g. limiting the number of records, or excluding specific details of records. 
The access of data consumers to these sources is accurately recorded, which allows for auditing and inspection of the intended usage of the data.

One important aspect of the system is the trade-off between the controlled environment, and the choice and offer of modelling and programming tools available to the researchers. We tackle this by providing the researchers a wealth of commonly used tools, the requirements for which were elicited by observing current practices. Further, the server environment offers a fast computing environment, with special hardware such as GPU computing available on demand.

\subsection{Active and passive security measures}

Active security measures aim at preventing access to data and other resources by unauthorised parties. In the DEXHELPP infrastructure, these include:

- Basic security provided by the Vienna University of Technology network

- A central authentication and authorisation schema, based on a directory service.

- A dedicated Virtual Private Network (VPN), which provides full encryption of all network transfer between the users and the infrastructure. The access to the VPN is protected with a two-factor authentication.

- Provisioning of access to data and resource only in the granularity required for the specific research question.

Passive security measures enable detection of inappropriate access and usage of DEXHELPP resources. They include

- A centralised and replicated logging of access to all resources, including services and data used by DEXHELPP partners and DEXHELPP customers.

- Embedding watermarks and personalised fingerprints into the data allows detecting data leakages, and to attribute the leakage to a specific user account (Figure2).

\subsection{Impact and effects}

With the DEXHELPP research services infrastructure, both data providers and data consumers profit from the facilitation in the exchange of and access to data, which dramatically speeds up the bootstrap process of a new research investigation. Due to the integrated data sharing and analysis infrastructure, data providers have the assurance that access to the data is accurately recorded and can be tracked and inspected also retroactively.

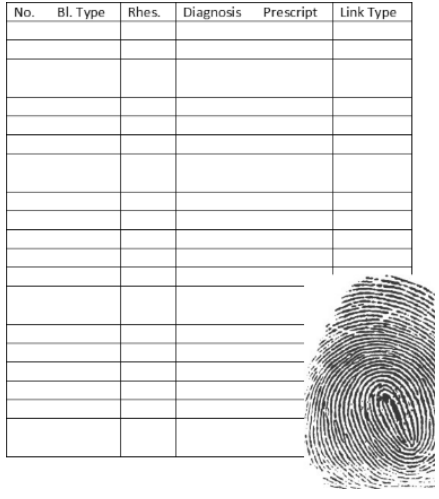

Figure 2: Active and passive security measures, like fingerprinting of data sets, are combined to unite privacy issues with possibilities for research and policy.

The usage of the secure research server infrastructure increases the trust among the project partners and increases the readiness for sharing data [3].

Data consumers benefit from an easier access to the data, the powerful computing environment, and, with security measures provided centrally as a service, can focus on their skills and actual tasks at hand, instead of spending time on data privacy and security concerns. The central provisioning of data and services also facilities the usage of tools that enhance the reproducibility of research investigations.

The research server is also a suitable environment for a third party to merge and link data sets from different sources, which otherwise would not be released, for example combination of data on social insurance with data on the labour market.

\section{Population Models}

Simulation models for aspects of the health system often require an underlying population model, which require substantial resources in the modelling process. In DEXHELPP a 'Generic Population Concept' (GEPOC) was developed to collect population data and implement population models to have them readily available.

Models are designed to be extended for healthcare aspects to make future projects faster and more efficient. Above that, this allows reproducible use of data and standardized transferability to other countries. As proof-of-concept, GEPOC was applied on various applications, like a vaccination model.

\subsection{Motivation}

Simulation models are a common way to perform analysis and planning in the healthcare system. 
Often, data does not directly provide answers and statistical analyses are insufficient. In the past DEXHELPP partners conducted several projects in Austria that included modelling and simulation. Agent-based models were developed to simulate the outcome of vaccinations for streptococcus pneumoniae and influenza.

Another model analysed cost effectiveness of screening for Abdominal Aortic Aneurysms. In another project, an agent-based model was developed to compare the costs of healthcare in different reimbursement systems. These models addressed completely different issues and answered completely different questions. However, each model needed a valid representation of Austria's population, and structures to simulate changes like births or deaths. Each project spent substantial resources to do the same thing again and again.

Therefore, the GEneric POpulation Concept GEPOC - should provide appropriate structures and model parts about the Austrian population. This should substantially decrease the effort of population modelling, and further the total effort of modelling projects in the future.

\subsection{Outline}

GEPOC generally consists of three parts: A handbook, model implementations, and data. The handbook contains a literature review on other population models, lessons learned from past projects, and comprehensive documentation of collected data and implemented models.

Model structures were implemented as agent-based models in Java and Python, and as a System Dynamics (SD) model in Anylogic. These combinations of implementations serve a wide range of projects conducted by DEXHELPP partners and customers. All models can simulate a population of humans with age and sex, including births, deaths, immigration, and emigration over a period of several years.

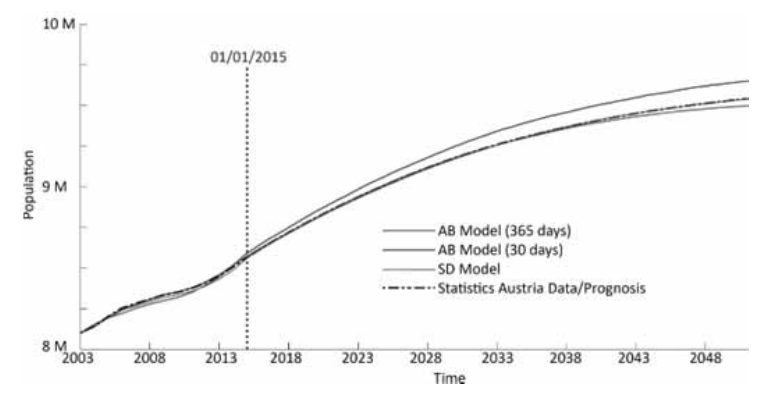

Figure 3: The resulting population of the AB model (with 365 and 30 days time steps) and the SD model compared to Statistics Austria's data.

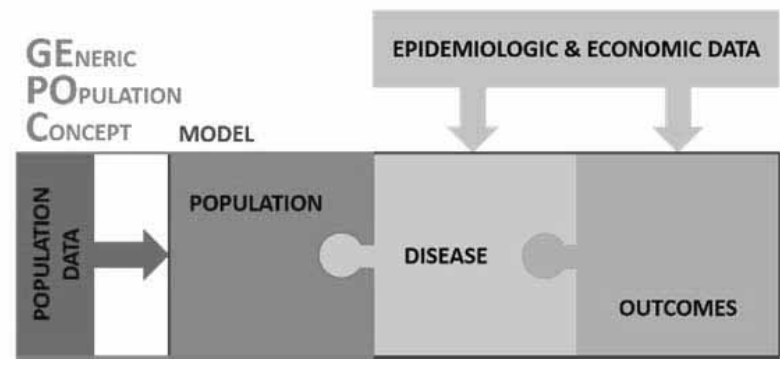

Figure 4: Models based on GEPOC can re-use population data to enrich these data sets with additional data.

The data about Austria's population, including births, deaths, immigration, and emigration, was collected from Statistics Austria. The data composes of data until 2014 and predictions until 2050. The total population's prediction is a result of Statistics Austria's own population model. The handbook describes the sources of this data, the pre-processing and storage format, and the mathematical calculations for model parameters.

\subsection{Validation}

Both the AB and the SD model were simulated for Austria's population from 2003 until 2050, using the initial population in 2003 and births, deaths, immigration, and emigration data for 2003-2050.

For validation, the resulting model population was compared to the total population data. Figure 3 shows that this works well. The SD model exactly resembles Statistics Austria's population prediction. The AB model contains small differences, which are acceptable and trace back to complex parameter calculation. Further research addresses this issue to minimize the differences.

\subsection{Impact}

The population models can reproduce Statistics Austria's data. Thus, they can be considered valid and serve as a basis for further modelling. This is the function that the pure population data cannot provide. The models are designed in such a way that they can easily be extended for diseases, treatments, and other healthcare interventions (Figure 4).

The population model, which serves as a necessary core of the model, can perform a standalone simulation of the population. Additional models were implemented $[4,5]$, e.g. an agent-based Python model to simulate vaccination rates in Austria for the Austrian Ministry of Health. 
Information was available about yearly vaccination numbers in Austria, disease outbreaks, as well as vaccination state of immigrants and refugees. As a result, the model shows vaccination and immunity states of Austria's population for each birth cohort. This analysis allows performing specific measures for children vaccinations and catch-up vaccinations to reach the health goals concerning the disease.

GEPOC provides population data readily available and allows using it in a well-defined reproducible way. The implemented models are designed to be used for healthcare issues and significantly reduce resources, time and cost of modelling projects. Data and models can easily be updated whenever new data is published. Data handling and models can even be transferred to simulate other countries without any major changes. Using the DEXHELPP Research Server and GEPOC provides so the possibility to implement fast and reproducible health system models.

\section{Atlas of Epidemiology}

In healthcare it is crucial to have a fundamental knowledge of the burden of diseases within the population. Therefore, one aim is to develop various Atlases, like an Atlas of Epidemiology to gain better insight on the epidemiological situation. Based on primary and secondary health care data, the goal was to present results in interactive charts and maps, comprehensible to experts and the public. The atlas builds a framework for rapid deployment of new data and results in a reproducible and efficient way. As first use case three methods based on two different databases for the estimation of diabetes prevalence in Austria were compared.

\subsection{Different sources - different results: methods applicable for the public}

Evaluating the burden of disease by estimating the prevalence or incidence of a disease within a population is usually conducted based on a specific data source. Often different data sources exist and lead to different - sometimes horrendous different - results, which makes decision making and further analyses difficult. Communication the different results to the public and explaining these differences seem to be challenging. Therefore, the partners of DEXHELPP developed concepts and a first use case of the Atlas of Epidemiology, a web-based tool, capable of tackling this challenge.
The tool developed here is generic and can be adapted to other questions of epidemiology of interest. The first use case compares three methods of estimating the prevalence of diabetes based on two different data sources. The success of this use case and the positive response by social security institutions and individuals, encouraged us to plan and (currently) develop a second use case: the prevalence of different diseases according to the ICD10 chapters based on the ATC-ICD project, where diagnoses are assigned to patients based on hospitalizations, sick leaves and prescription data.

The methods used for communicating the different results of the burden of disease to the public include interactive bar charts and heat maps of Austria for patients divided by region, gender and age together with descriptions of results. The user is encouraged to experiment with the different results.

\subsection{Successful implementation: use case Diabetes}

The first use case for estimating prevalence of diabetes was successfully implemented based on two different data sources:

I. reimbursement data 2006/2007 (GAP-DRG);

II. national routine health surveys (ATHIS) for 2006/2007;

with three different methods (see Figure 5):

1. ATC-ICD statistically relates pseudonymized data on medications to data on diagnoses from hospitalizations and sick leaves.

2. Experts: medical experts assign specific medications to diabetes diagnoses. Patients with these medications are identified together with hospitalized diabetes diagnosed patients in GAP-DRG.

3. ATHIS a sample of 15.000 persons was questioned if they a) ever had diabetes and b) were treated against diabetes in the last 12 months. Results are projected onto the Austrian population.

4.

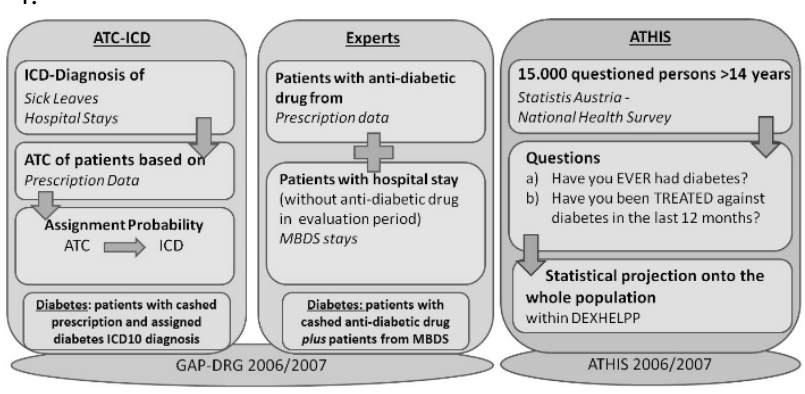

Figure 5: Different Methods and Different Data Sources for the Use Case of Diabetes Prevalence. 
Patients are divided by 10-year age-classes, gender and region. For the publicly online framework (http://www. dexhelpp.at/de/epidemiologie-atlas/), implemented in html and javascript, pre-processed data in different granularity is required and used.

\subsection{Impact and effects}

The atlas was publicly presented online to decision makers, health care providers and social security systems [6]. The feedback was very positive to our novel approach and has been incorporated. Maps of Austria represent the prevalence of diabetes for each method and granularity level. The difference of the methods can be seen by clicking on the next map. For different ageclasses (resp. different gender) the three methods can be compared directly within a bar chart. The technology for a rapid deployment of new data is now developed which has a major impact on estimating the burden of disease, since it is now possible in a fast an efficient way which allows focusing more on the intervention than the representation of epidemiology.

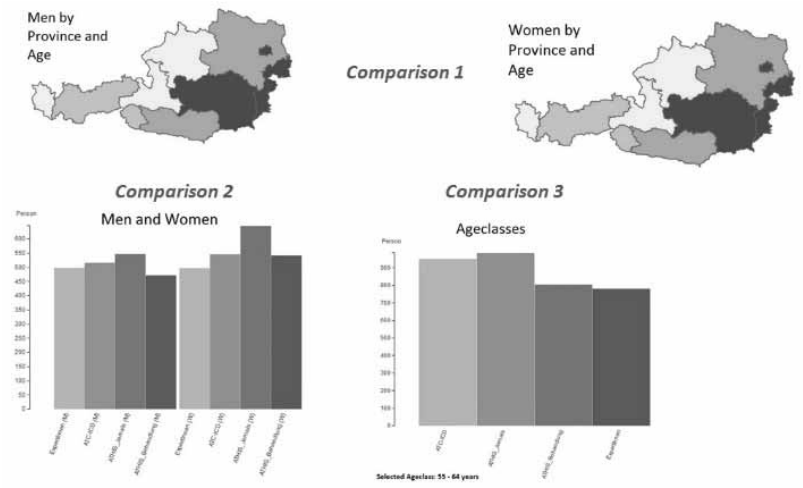

Figure 6: Interactive Comparison methods for Use Case of Estimating Diabetes Prevalence based on different data sources.

Besides depicting disease prevalence, the Atlas of Epidemiology also allows to quickly visualize health care service data and results of simula-tion models, which is important for decision makers and the communication to the public. Results of the ATC-ICD project on the prevalence of different diseases based on ICD9 diagnoses and medication data were also integrated in an aggregated form.

\section{Big Data and System Modelling}

Three examples for Data Processes, Modelling \& Computation and possible Interfaces were described above.
These are only examples for methods already implemented in DEXHELPP and many other activities in Europe and around the world. The near future will bring integrated solutions with Deep Learning, Network Models and many more. E.g. based on modules like GEPOC and the DEXHELPP Research Server, virtual clinical trials can be implemented soon.

\section{References}

[1] Habl C, Renner A, Bobek J, Laschkolnig A. Study on Big Data in Public Health, Telemedicine and Healthcare. Report / Study, 16.12.2016, Directorate-General for Health and Food Safety, Directorate B-Health systems, medical products and innovation.

[2] Salcher M. Connecting the Dots: Putting Big Data to work for Health Systems. Eurohealth; Quarterly of the European Observatory on Health Systems and Policies Vol.23, No.1 2017; S.3-6

[3] Pröll S, Rauber A. Enabling Reproducibility for Small and Large-Scale Research Data Sets. D-Lib Magazine, 23 (2017), 1/2; 6 S.

[4] Bicher M, Urach C, Zauner G, Rippinger C, Popper N. Calibration of a Stochastic Agent-Based Model for ReHospitalization Numbers of Psychiatric Patients. Proc. 2017 Winter Simulation Conference, Chan W K V, D’Ambrogio A, Zacharewicz G, Mustafee N, Page S (eds.); IEEE, CFP17WSC-USB (2017), ISBN: 978-15386-3428-8; $12 \mathrm{~S}$.

[5] Schneckenreither G, Popper N. Dynamic Multiplex Social Network Models on Multiple Time Scales for Simulating Contact Formation and Patterns in Epidemic Spread. Proc. 2017 Winter Simulation Conference, Chan W K V, D’Ambrogio A, Zacharewicz G, Mustafee N, Page S (eds.); IEEE, CFP17WSC-USB (2017), ISBN: 978-1-5386-3428-8; $12 \mathrm{~S}$.

[6] Glock B, Endel F, Endel G, Sandholzer K, Popper N, Rinner C, Duftschmid G, Filzmoser P, Mert M C, Holl J, Wagner-Pinter M. How sick is Austria? - A decision support framework for different evaluations of the burden of disease within the Austrian population based on different data sources. Int. Journal of Population Data Science, 1 (2017), 1; S. 92.

Note. The contribution was supported by DEXHELPP Decision Support for Health Policy and Planning, which is funded by the Austrian Ministries for Innovation, Technology, Science, Research and Economy as well as the state of Vienna via COMET (Competence Centers for Excellent Technologies). The COMET program is processed by FFG (Austrian Research Promotion Agency). 


\title{
How Modelling and Simulation Can Benefit the Most from Machine Learning
}

\author{
Matthias Wastian*, Dominik Brunmeir \\ dwh Simulation Services, Neustiftgasse 57-59, 1070 Vienna, Austria; *matthias.wastian@dwh.at
}

SNE 27(4), 2017, 209-212, DOI: 10.11128/sne.27.sn.10397

Received: December 2, 2017, Revised: December 18, 2017, Accepted: December 22, 2017

SNE - Simulation Notes Europe, ARGESIM Publisher Vienna, ISSN Print 2305-9974, Online 2306-0271, www.sne-journal.org

Abstract. This article is about possible intersections between modelling and simulation and machine learning. It introduces some basic ideas of machine learning and shows how the can be applied onto traditional modelling and simulation processes in such a way that both approaches benefit as much as possible from each other. New developments and advancements in various areas allow for new techniques which may help in understanding complex dynamic systems.

\section{Introduction}

Before we talk about what we believe are the most interesting applications of machine learning methods in modelling and simulation nowadays, we shortly introduce some basic definitions. Then we give an overview about how machine learning methods can help to optimize or approximate models as well as how machine learning can be used within a model to improve it, e.g. by making agents in an agent-based model more intelligent.

\section{Fields of Interest}

\subsection{Machine Learning and Data Science}

Tom M. Mitchell provided a widely quoted, formal definition of the algorithms studied in the machine learning field: "A computer program is said to learn from experience $E$ with respect to some class of tasks $T$ and performance measure $P$ if its performance at tasks in $T$, as measured by $P$, improves with experience E. " [1]
Machine learning and statistics are closely related fields. According to Michael I. Jordan, the ideas of machine learning, from methodological principles to theoretical tools, have had a long prehistory in statistics. He also suggested the term data science as a placeholder to call the overall field.

Typical machine learning tasks include:

1. Mostly supervised learning tasks:

- Classification: building models to predict class labels for new observations

- Regression: building predictive models for continuous observations

2. Mostly unsupervised learning tasks:

- Clustering: segmenting data into natural subgroups

- Dimensionality reduction: mapping highdimensional data into a lower-dimensional space, e.g. word embeddings.

Commonly used machine learning algorithms are listed below:

- Nearest neighbors

- Decision trees

- Artificial neural networks

- Support vector machines

- Bayesian networks

- Hierarchical clustering

- Genetic algorithms 


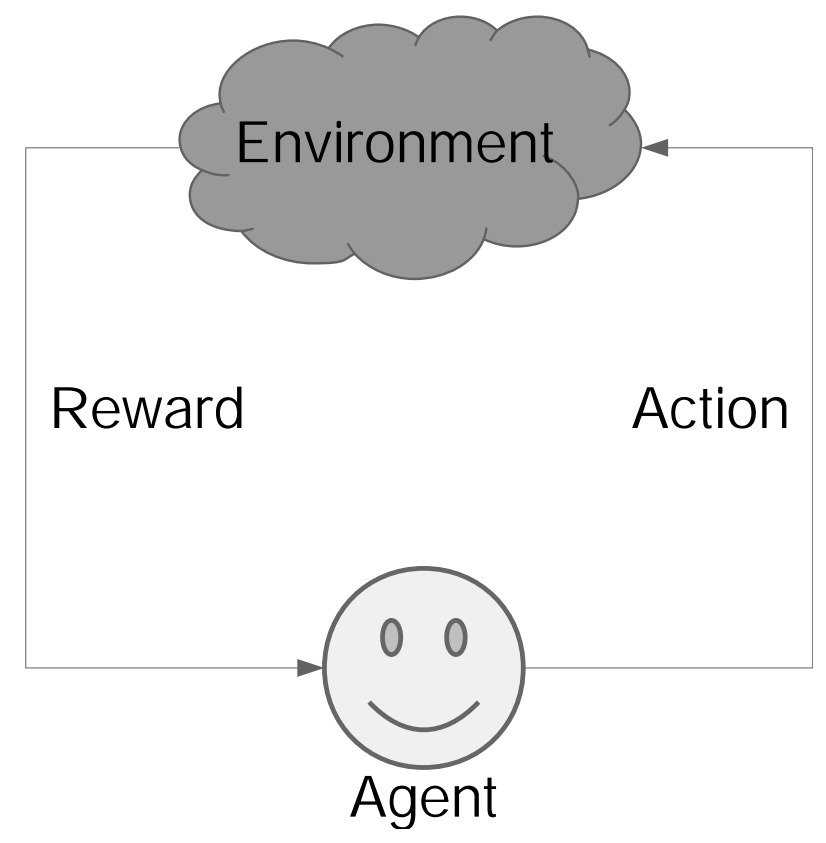

Figure 1: Reinforcement Learning Loop

\subsection{Reinforcement Learning}

Reinforcement learning is a part of machine learning that focuses on learning optimal control in a partially observable markov decision process. This means teaching a software agent an optimal decision policy using rewards and penalties as signals. Richard S. Sutton [8] classifies it as neither supervised nor unsupervised but as an own branch of machine learning. The main difficulty is to balance the need for exploration and exploitation. Exploration focuses on finding new ways to gain rewards, while exploitation is necessary to utilize known ways to gain rewards. A reinforcement learning problem generally consists of the following parts:

- policy

- reward signal

- value function

- model (optional)

The policy defines the action an agent takes in a given state. This is what an agent learns in a classical reinforcement learning problem. The reward signal gives feedback to the agent after each time step. It rewards or punishes the action taken by the agent according to its policy. The agent's goal is to maximize the rewards. The value function may be described as the long-term goal of the agent. It is the expected reward an agent may achieve in the future. This is in contrast to the reward signal, which only gives the immediate motivation for an action. As a fourth element we can add the model, which is used for planning. It describes the environment and the change of state. This is entirely optional, but allows the use a different set of algorithms.

\subsection{Deep Learning}

Deep learning is an established approach to learn data representations with the help of deep neural networks. In contrast to "shallow" neural networks deep ones consist of many layers between the input and the output layers, called hidden layers. Deep learning is dealing with learning multiple levels of representation and abstraction that can be discovered in structured data as well as in unstructured data [10]. Advancements in hardware and learning algorithms have made it feasible to employ deep learning architectures in a multitude of machine learning tasks, for example computer vision, speech recognition, natural language processing, audio recognition, social network filtering, machine translation or other classification tasks. Modern architectures achieve a superhuman performance in many of those.

\subsection{Deep Reinforcement Learning}

The idea of using deep neural networks with reinforcement learning is not new, but advancements in computing power allow a more liberal use of these techniques. We may employ deep neural networks to approximate each part of a reinforcement learning Problem (policy, reward function, value function) as needed. With naive approaches this is highly unstable but recent approaches (e.g. [9], [4]) have made many improvements and achieved a more stable learning without exploding or vanishing gradients. Systems using deep reinforcement learning show promising ability to generalize learned behavior and once trained can be used in similar models and achieve good results.

\section{Macroscopic Usage of Machine Learning: Simulation Optimization}

The term simulation optimization refers to the search for specific hyperparameter settings to a stochastic simulation such that a target objective, which is a func- 
tion of the simulation output, is minimized without loss of generality [11]. As complex models that are build nowadays might include an extremely large number of possible hyperparameter settings, machine learning methods can be very useful to find an adequately good hyperparameter setting for the model of interest.

\section{Usage of Machine Learning within a Model}

\subsection{Agent-Based Models}

Agents are defined as autonomous entities that interact with themselves and the environment. This interaction may be defined through simple rules and may lead to interesting emergent behavior. The rules often have a physical, biological or sociological background.

A well-known example model are the LotkaVolterra equations. They describe the population of interacting species in a dynamic system. Let $x$ be the number of prey and $y$ the number of predators, then we may describe the change of population as follows:

$$
\begin{aligned}
& \frac{d x}{d t}=\alpha x-\beta x y \\
& \frac{d y}{d t}=\delta x y-\gamma y
\end{aligned}
$$

$\alpha, \delta$ are reproduction parameters of the prey and predators, while $\beta$ is the contact rate (can be interpreted as prey consumption rate) and $\gamma$ is the loss of population of the predator species.

If we want to describe this as an agent-based model, we first define two different classes of agents with differing behavior. While both agents move around randomly, the predator agent eats a prey if it comes within a certain radius $\hat{\beta}$ and the predator dies with a certain probability $\hat{\gamma}$. Within fluctuations due to stochastic effects, this model behaves almost equally to the classical model.

From this point of view it is easy to track and analyze the behavior of a single agent. It is also easy to add certain constraints to the environment where the agents live and so generate new insights.

\subsection{Prediction}

Many models include some predictive element and may benefit from machine learning for doing so. If we consider the model of an icehockey game, the agent im- proves his performance if he skates where the puck is going to be, because he is able to accurately predict the trajectory of the puck, based on data from real pucks.

\subsection{Reinforcement Learning}

As reinforcement learning deals with agents learning a certain behavior, it fits naturally in improving agentbased models [7].

Behavioral Approximation. Many behavioral rules are based on data, collected in real world environments. These rules can be very complex and often cannot be sufficiently simplified to be summarized in a rule system. Deep reinforcement learning can be used to approximate the behavior of the agents from data without any prior knowledge [2] [3].

Behavioral Optimization. Another aspect of employing deep reinforcement learning to agent based modeling is training the agents to utilize an optimal behavior. If we consider the predator-prey model, a single prey agent may survive longer if it evades the predator. If we let a prey learn, such a behavior this can lead to swarm or herd building as emergent behavior [6] [5].

\section{Model Approximation}

Machine learning can also be very helpful to modelers for approximating already existing models. The universal approximation theorem [12] states that a feedforward neural network with a single hidden layer containing a finite number of neurons can approximate any continuous function with arbitrary accuracy on compact subsets of $\mathbb{R}^{n}$ under very mild assumptions on the activation functions used. One of the first versions of the theorem was proven by George Cybenko for the case of sigmoid activation function [13]. In an extension by Kurt Hornik this was shown for a larger set of activation functions [14].

Universal Approximation Theorem. Let $\phi$ be a nonconstant, bounden and monotonically increasing continuous function, $I_{m}$ the $m$-dimensional unit hypercube and $C\left(I_{m}\right)$ the space of continuous functions on $I_{m}$. For any $\varepsilon>0$ and any function $f \in C\left(I_{m}\right)$ there are an integer $N$, real constants $v_{i}, b_{i} \in \mathbb{R}$ and real vectors 
$w_{i} \in \mathbb{R}^{n}$ with $i=1, \ldots, N$ such that:

$$
\left|\sum_{i=1}^{N} v_{i} \phi\left(w_{i}^{T} x+b_{i}\right)-f(x)\right|
$$

This still holds when replacing $I_{m}$ with any compact subset of $\mathbb{R}^{m}$.

How this approach can be implemented to enlarge an already existing model is shown by the following example.

\subsection{City Quarters with Optimised Solar Hybrid Heating and Cooling Systems}

In order to be able to share potential energy surpluses between buildings, a good starting point for a city quarter model like in this project (FFG number 845168) was to build physical models for certain building types with specified configurations (e.g. regarding size, the domestic heat water demand, a potential photovoltaic unit, a potential cooling system). Simulation runs for one building are computationally feasible, but the computation times for combining a lot of buildings to a city quarter model or maybe even a model of a whole large city really skyrocket. Thus the approach was to approximate the physical building models with computationally cheaper data models based on machine learning algorithms like extremely randomized trees [15], a special kind of random forests.

Machine learning based model approximation allowed to transfer the original model to larger scales.

\section{References}

[1] Mitchell, Tom M., Machine Learning. McGraw-Hill, Inc., New York, NY, USA. 1997.

[2] Wang et al, Deep Reinforcement Learning of Cell Movement in the Early Stage of C. elegans Embryogenesis. arXiv:1801.04600

[3] Bloembergen et al, Evolutionary Dynamics of Multi-agent Learning: A Survey. J. Artif. Int. Res. May 2015; 53: 659-697.

[4] Mnih et al, Playing Atari with Deep Reinforcement Learning. arXiv: 1312.5602

[5] Yang et al, An Empirical Study of AI Population Dynamics with Million-agent Reinforcement Learning. arXiv: 1709.04511

[6] Olsen, Megan M. and Fraczkowski, Rachel, Co-evolution in predator prey through reinforcement learning. Journal of Computational Science; 2015
[7] Stankovic, M., Multi-agent reinforcement learning. Symposium on Neural Networks and Applications. 2016.

[8] Sutton, RS, Barto AG Reinforcement Learning: An Introduction. 4th Edition. Publisher: MIT Press; 2018

[9] Mnih et al, Human-level control through deep reinforcement learning. Nature 2015. 518: 529-533. doi: 10.1038/nature14236

[10] Tolk, Andreas, The Next Generation of Modeling \& Simulation: Integrating Big Data and Deep Learning. Summer Simulation Multiconference 2015, Chicago, IL.

[11] Amaran, Satyajith et al., Simulation Optimization: A Review of Algorithms and Applications. Annals of Operations Research, May 2016, Volume 240, Issue 1, pp 351-380.

[12] Balázs Csanád Csáji, Approximation with Artificial Neural Networks. Faculty of Sciences. Eötvös Loránd University, Hungary. 2001.

[13] Cybenko, G., Approximations by Superpositions of Sigmoidal Functions. Mathematics of Control, Signals, and Systems, 2 (4), 303-314, 1989.

[14] Hornik, Kurt, Approximation Capabilities of Multilayer Feedforward Networks, o. Neural Networks, 4(2), 251-257. doi:10.1016/0893-6080(91)90009-T, 1991.

[15] Geurts, P. et al. Extremely Randomized Trees. Machine Learning, 63(1), 3-42, 2006. 


\title{
A Critical Overview of Modelling and Simulating Methods for Complex Dynamic Systems
}

\author{
Peter P. Groumpos \\ Laboratory of Automation and Robotics, Department of Electrical and Computer Engineering, \\ University of Patras, Greece; *groumpos@ece.upatras.gr
}

SNE 27(4), 2017, 213 - 223, DOI: 10.11128/sne.27.on.10398 Received: December 15, 2017; Revised: December 18, 2017; Accepted: December 22, 2017

SNE - Simulation Notes Europe, ARGESIM Publisher Vienna, ISSN Print 2305-9974, Online 2306-0271, www.sne-journal.org

Abstract. Nowadays, practical dynamic systems have become more and more complex. The concept of Complex Dynamic Systems (CDS) arises in many scientific fields, technological areas and everyday's problems. The fundamental basics of complex dynamic systems are briefly presented and discussed. Modelling and simulating complex dynamic systems is a very difficult and challenging task. Different modelling approaches basically focus on the interaction between (microscopic) subsystems and the emergence of new qualities at the (macroscopic) system level. However these models are not sufficient to describe the dynamic behavior of today's systems. Similarly despite the numerous Simulation methods and large number of simulation software tools many of today's challenging problems cannot be studied appropriately and provide satisfactory answers. It seems that the uncertainty and fuzziness been inevitably present on these systems make the problems more difficult. The new models of Fuzzy Cognitive Maps are proposed as a new modelling approach for CDS. The need for new advanced models and new simulation methods is apparent taking into consideration the presence of human nature in all system processes, as well as the fuzzy and uncertaint environment. Some conclusions and future research directions are provided.

\section{Introduction}

In this overview paper the modeling and in parallel simulation methods been used in analyzing and studying today's Complex Dynamic Systems (CDS) is carefully, critically and wisely reviewed. The science of complex dynamical systems is a multidisciplinary field aiming at understanding the complex real world and its dynamic behavior that surrounds us.
Examples of these systems are: energy networks in distributed power systems with different renewable energy sources, neural networks in the brain that produce intelligence and consciousness, artificial intelligence systems, environmental systems, swarm of software agent, traffic patterns, biological systems, social and economic systems and many other scientific areas can be considered to fall into the realm of complex dynamical systems.

The title of the paper is very broad and overambitious to cover all issues of modelling and simulation methods for understanding the behavior of CDS. It is impossible to cover extensively and in a total exhaustive way the title of this paper. In order to accomplish this objective, we need an encyclopedia with many volumes.

The paper is rather provocative and by raising many challenging questions can be a sparkling fire that will drive the reader to unexpected avenues. Thus there are not given many details on most crucial and important issues. I leave the reader to search for the answers to the very important, critical, serious and challenging problems that the whole world is facing. There is an enormous literature with books, papers and many other sources that the reader should consult to find answers to the questions been raised in this paper.

Another very valuable and useful source for seeking answers is the International Journal SNE (Simulation Notes Europe). This SNE journal under the strong and enthusiastic editorial leadership of Felix Breitenecker and of the SNE Editorial Board, is an excellent source for following all the recent Research and Academic developments in the broad scientific area of Simulations. So once again I encourage the reader of this paper to focus on the questions been raised here and search for the new and innovative solutions that our academic and scientific communities so much are in need. 


\section{Challenging Issues in Modelling and Simulating Complex Dynamic Systems}

Modelling of a system is a fundamental work which is always the starting point for the understanding, designing, control, optimisation, and implementation of any physical and/or human made system. This is also the case for Complex Dynamic Systems (CDS). However, the last 30-40 years, Complex Dynamic Systems present many problems in mathematical modelling, control implementation, simulation methods and philosophical foundations. But why? What are the reasons? Do we need to be wiser? Certainly YES.

Complex Dynamic Systems (CDS) comprise of collections of many heterogeneous entities, which interact with other entities and their environment, which usually are having a lot of uncertainties, fuzziness, ambiguities and structural complexities. Interactions among subsystems are localized, often seeking autonomy and self-organizing, while most of the times are nonlinear, dynamic, fuzzy and possibly chaotic. The study of CDS represents a new approach to science that investigates how relationships between parts give rise to the collective behaviors of a system and how the system interacts and forms relationships with its environment. CDS have some specific characteristics, among which are: uniqueness, weak structuredness of knowledge about the system, incompleteness of its dynamic behavior, antagonism among different agents, the composite nature of system and heterogeneity of elements composing the complex system. Furthermore decisions must be made ensuring the smooth, reliable, stable and cost effective operation of each of the subsystem as well the whole CDS. There are many books on this modelling issue of Complex Dynamic Systems (CDS) [1]-[8].

Another important feature of CDS is that a network structure, including hierarchical one, self-organization can amount to:

(1) disconnecting certain constituent nodes from the system,

(2) connecting previously disconnected nodes to the same or to other nodes,

(3) acquiring new nodes,

(4) discarding existing nodes,

(5) acquiring new links,

(6) discarding existing links,

(7) removing or modifying existing links.
In addition today's society's challenging problems demand CDS to have a number of Properties-Abilities (P-A) such as: co-evolution, anticipation, adaptation, cooperation such as swarming, intelligence, consciousness, genetic regulation - homeostasis, development, disease, cascading failures in electrical grid, invasiveness in plants, hurricanes and self-repairing materials, cognition, emergence, self-evaluation and organization, robustness and wisdom.

All these collective dynamics of a CDS give rise to 'Emergent Evolution Properties-Abilities' (E.E.P-A) at higher scales in space and/or time. Under such conditions, the key problem of Complex Dynamic Systems and control theory consists in the development of methods of qualitative analysis of the dynamics and behavior of such systems and in the construction of efficient control algorithms for their efficient operation. In a general case, the purpose of control is to bring the system to a point of its phase space which corresponds to maximal or minimal value of the chosen efficiency criterion.

Another one of the main and actual problems in the theory of complex dynamical systems (CDS) and control sciences is a solution of 'ill-posed, weakly- and poorlystructured and weakly- formalizable complex problems' associated with complex technical, organizational, social, economic, cognitive and many other objects, and with the perspectives of their evolution. One more critical aspect that must be seriously taken into consideration is that the human presence in all CDS is inevitable. This problem is very critical in studying CDS because we are actually dealing with Dynamic Systems and want to understand their long-term qualitative behavior. However the focus is not on finding precise solutions to the equations, which most of the times are not well mathematically defining the complex dynamic system. Such a search is often hopeless.

The solutions been sought would rather answer questions like

- 'Will the CDS settle down to a steady state in the long term, and if so, what are the possible steady states?'

- 'Are the steady states, precise or are fuzzy and ambiguous?', or

- 'Does the long-term behavior of the system depend on its initial condition?' or

- 'How important is to depend on the knowledge of experts and if so, how many experts should be consulted?' or

- 'Does the past history of the behavior of the CDS influences its long-term behavior' or 
- 'What is more important: correlation or causality between the states?' or

- 'How sufficient are the mathematical models to predict the long term-behavior of the CDS?' or

- 'If not what other alternatives do we have?'.

A more fundamental question:

- 'Is there a difference between systems and mathematical models?'.

A system can be described in terms of its components and their interactions. A system is a group of related parts that make up a whole and can carry out functions its individual parts cannot. On the other hand, models are be used to represent systems and their interactions - such as inputs, processes and outputs - and energy and matter flows within systems. Systems may interact with other systems; they may have sub-systems and be a part of larger complex systems. On the other hand, models are limited in that they only represent certain aspects of the system under study. Thus, the crucial point in analyzing and studying the dynamic behavior of complex systems is to clearly understand this fundamental difference, and analyze and study the different modelling approaches for CDS.

\section{An Overview of Mathematical Approaches for Modelling CDS}

The issue of mathematical modelling of CDS is not an easy task. In addition, many times the difference between the system and its mathematical representation of a process or operation of a complex system is not clear. This is very critical. Systems and System Models are useful in science and engineering because the world is complex, so it is helpful to isolate a single system and construct a simplified model of it. In order to do this, scientists and engineers imagine an artificial boundary between the system in question and everything else. They then examine the system in detail and using a variety of modelling methods a mathematical model is generated.

It is different to model a physical or human made system with Ordinary Differential Equations (ODE) than to represent its operation through for example with a Taylor series approximation model. Some would argue that most models are often an approximation of the physical properties and dynamic characteristics of a system. This is partially true however, the validity of the classification is limited to the accuracy with which the particular model is described and moreover the way is "behaving" in the real world. In general systems are classified according to the mathematical representation (equations or not) used to describe them and how they are prepared. It is not the objective of this paper to discuss extensively all types of classifications with all their ramifications.

Rather we will single out that type of systems, which are within the scope of the title especially with respect to simulation modelling and analysis. Furthermore the classical classification just 60-70 years ago, started with continuous vs. discrete systems. This to me is a very important issue. I argue that the classification of systems is different than the mathematical model representation of the corresponding system. I believe it was wrong to start this way the classification. I believe that the first classification is deterministic vs. stochastic models. In deterministic models, the output of the model is fully determined by the parameter values and the initial conditions. Stochastic models possess some inherent randomness. The same set of parameter values and initial conditions will lead to an ensemble of different outputs. The next classification is linear vs. nonlinear systems. The next classification static vs. dynamic systems. Then continuous vs. discrete systems and then, time varying vs. time invariant.

The important remark here is that for each system classification there should be a similar modelling classification. However this is not always the case and easy to be done. For example a system is non linear however the mathematical model could be a linear approximation. This further complicates the appropriate simulation model and software tool that should be used to simulate the dynamic behavior of the system.

\section{Basics of Simulation M odelling and Analysis}

Simulation is much more meaningful when we understand what it is actually doing. Understanding how simulation works helps us to know whether we are applying it correctly and what the output results mean. But what is simulation? And what is its relation to modelling? Simple and easy questions but difficult to comprehend the answers to them and more important to understand how to use both (modelling and simulation) to analyze and understand the behavior of Complex Dynamic Systems (CDS). Many books have been written that give thorough and detailed discussions of the science of simulation [9]- 
[14]. Let enlighten ourselves with a few definitions and methods for Simulations. To simulate is to try to duplicate the characteristics of a real system and to approximate its dynamic behavior Simulation is one of the most widely used decision modeling techniques. Before the coming of the digital age, only analog computers existed and analog simulations were developed and used. In other words, till digital simulations came to the academic and scientific communities after the 1950ies.

In order to understand the significant difference and the tremendous push that the digital computer provided to the simulations a few examples of analog computers and their applications should be mentioned. The technology of the ubiquitous operational amplifier - now an indispensable component of every control system - was originally invented and perfected for analog computing. Columbia professor John Ragazzini invented the term "operational amplifier" and described the state-of-the-art electronic analog techniques in his landmark paper [15].

The simulation of guided missiles in the late 1950ies pushed the limits of both analog and digital computing. Digital computers were not yet fast enough to permit hardware-in-the-loop testing of the real-time control systems, but analog computers did not have the accuracy or dynamic range to simulate the long-range trajectories. Electronic analog-to-digital converters, another now-indispensable technology, were first commercialized for the purpose of interfacing analog computers with digital machines. These hybrid computers bridged the gap, using analog techniques to simulate the vehicle with its control surfaces and using digital techniques to calculate navigational coordinates.

As a tool, the early differential analyzer was more than a general-purpose, differential-equation solver. It was an educational tool and a research touchstone. The history of control is entwined with the history of analog computing. Many of the tools, technologies, and theories of control were enabled by, or are directly descended from, mechanical and electronic analog computers. Ana$\log$ computers played a key role in enabling the simulation of control systems for several decades, leading to a better understanding of theory and better designs in practice.

Today Simulation imitates the operations of a system or process, usually via computers. We must emphasize: what's being simulated is the system, figure 1 . To study the system, often make assumptions/approximations, both logical and mathematical, about how it works.

These assumptions form a model of the system. If model structure is simple enough, could use mathematical methods to get exact information on questions of interest - analytical solution.

But most complex systems require models that are also complex in order to be closer to the real system. Thus, it must be studied via simulation - evaluate model numerically and collect data to estimate model characteristics and subsequently to understand the dynamic (or static) behavior of the system.

\section{Modelling and Simulation Methods for Complex Dynamic Systems}

As was explained above simulation models or methods can be developed to study the behavior of Complex systems. However the task is very complicated because all scientific areas have CDS. Even more difficult is the task, to approach each thematic area and perform the modelling and simulation task.

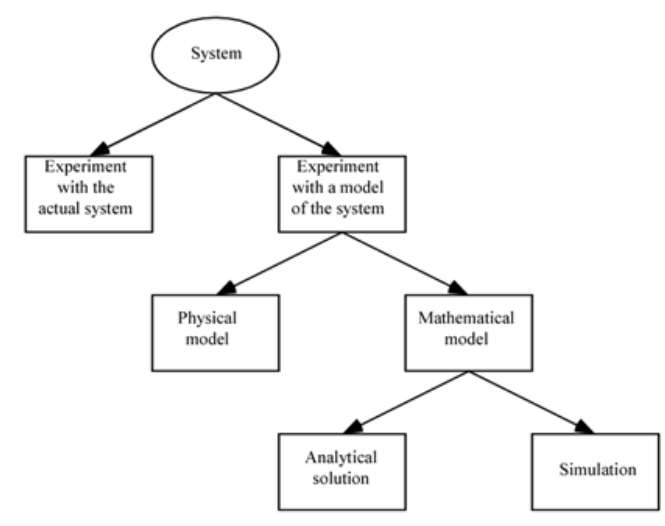

Figure 1: A flow chart for addressing the steps of modeling and simulation of CDS.

So for different complex scientific areas a good number of mathematical models and the corresponding software tools have been developed. Let us see some of them.

ENGINEERING SYSTEMS: for these systems the following four (4) generic mathematical approaches for modelling any CDS are proposed:

1) the Ordinary Differential Equations

2) the impulse function response

3 ) the transformation methods and

4) the state space representation. 
The main and very important point here is that all four generic approaches are modelling exactly the same physical or human made system. Knowing the one all the other three can be generated. This, four (4) generic mathematical approaches in modelling the same complex system is presented for first time here. However, for the purpose of this paper we will review in the more classical ways the modelling problem of CDS.

The focus is on modeling techniques that can glue together subsystems from diverse physical domains. Thus it depends also on the sub-scientific area that we are trying to solve a problem. For example, the concept of energy (or power), which is universally valid across many physical domains, is the right tool for combining electrical, mechanical, hydraulic, pneumatic, thermal and thermodynamic systems. However, some of the developed methods using the concept of energy is at least partially useful in the domains where the concept of energy is not so useful such as socio-economic systems. In total three groups of modeling techniques, which are based on the concept of energy have been develope. [2], [4], [8].

Analytical methods based on the Lagrangean and Hamiltonian functions well known from the studies in theoretical physics and/or mechanics, object-oriented modeling as an alternative to the more widespread blockoriented modeling, and last but not least an intuitive graphical technique known as bond graph modeling. Whichever methodology is followed to create the mathematical model, of the ways to analyze it is a numerical simulation, that is, numerical solution of the corresponding differential or differential-algebraic equations. Then appropriate simulation tools will be developed on the basics of numerical techniques for differential and differential-algebraic equations with the objective to understand the basic issues such as approximation errors, numerical stability and suitability of the common methods for different classes of models.

Thus the task of modelling and simulation methods for CDS is not a simple task. There have been developed many simulation models for engineering complex systems. We can have mentioned here the two most common simulation tools: MATLAB and Simulink.

MATLAB is the high-level language and interactive environment used by millions of engineers and scientists worldwide. It lets you visualize ideas across disciplines including signal and image processing, communications, control systems, and computational finance. Simulink is a block diagram environment for multi-domain simula- tion and Model-Based Design. It supports simulation, automatic code generation, and continuous test and verification of embedded systems. Simulate dynamic systems leveraging graphical editors, customizable block libraries, and solvers for modeling. An important remark is that when you use MATLAB and Simulink together, you combine textual and graphical programming to design a more powerful simulation software tool. The interesting reader can find many useful materials for both simulation software tools.

BUSINESS AND ECONOMICS: Business and Economic sectors there are many models and software tools. One of the most known simulation model is the Monte Carlo Simulation model. For historical reasons it was developed during the Second World War. Before the Monte Carlo method was developed, simulations tested a previously understood deterministic problem and statistical sampling was used to estimate uncertainties in the simulations. Monte Carlo simulations invert this approach, solving deterministic problems using a probabilistic ana$\log$ and solving the problem probabilistically. These simulations were used in investigating radiation shielding and the distance that neutrons would likely travel through various materials.

They were a vital simulation in the Manhattan Project, (of the atomic bomb), even though the computational tools were underdeveloped. During their work on the Manhattan Project John von Neumann and Stanislaw Ulam named the method after the Monte Carlo Casino in Monaco. Just for the Monte Carlo Simulation approach quite a few software tools have been developed: GoldSim, TRIDYN, Fluka, Maestro, PSAT, C++ to mention a few and just for one simulation approach. Please also note that the software to be used depends on the application working on.

MEDICAL SYSTEMS: Simulation enables healthcare systems and processes to be tested in a safe, virtual environment without risk to patients and staff - supporting organizations to make better decisions, improve systems of care and maximize resources. There are simulation tools: SIMUL8. PSF, Apollo, CSSC (for clinical studies), UroSim, LapSim (Laparoscopy), FlexSim, VisualSim (for ophthalmology) and many others.

IMPORTANT REMARK: it is impossible to cover extensively and in a total exhaustive way the title of this paper. In order to accomplish this objective, an encyclopedia with many 'scientists' and many volumes is 
needed. So once again I encourage the reader of this paper to focus on the questions. The author trying the above analysis believes that he contributes a small piece of scientific work, aiming in developing a new uniform theory of Intelligent and Cognitive Systems theory. Such a new theory is badly needed as will be explained below.

\section{Do We Need New Mathematical Modelling and Simulation Tools for CDS?}

In the previous sections we have seen that numerous mathematical modelling approaches and similarly a large number of simulation software tools for the Complex Dynamic Systems have been developed. However, we are facing every day numerous unsolved, critical and challenging problems. In Section 1, many questions where raised regarding the issues of modelling of CDS.

ONE VARY BASIC QUESTION IS: ARE WE HAPPY WITH TODAYS' MATHEMATICAL MODELLING AND SIMULATING APPROACHES?

If yes then why we keep failing to solve the basic problems of our societies? The poor children of the third world countries do not have clean drinking water! Even worst: we are not capable to solve the many problems our world is facing but instead with our actions we generate even more problems, which further complicate the whole problem. Our ignorance and/or our denial to realize the threats that we ourselves create with our actions, to preserve our environment. We are the ones that waste the energy sources foolishly and/or do not utilize better the renewable energy sources (RES).

If we are not happy what are we doing to correct our past mistakes? Have we learned from history or do we insist to ignore it?

More questions can be raised which are relevant to this paper. Do all today's models and associated solutions provide satisfactory and working conditions to the everyday behavior of Complex Dynamic Systems?

Indeed, there is a good and large number of situations or problems where today's models and solutions fail to give satisfactory answers. Making decisions in the area of Complex Dynamic Systems often strains our cognitive capabilities. Uncertainty and related concepts such as risk, fuzziness and ambiguity are prominent in the research and accompanied literature on Decision-Making.
- What are the best models?

- Do all models have detailed software tools that can adequately simulate their behavior?

- What is intelligence?

- What is wisdom?

- Is Intelligent Control related to Cognitive Control?

- Do all today's models and associated solutions provide satisfactory and working conditions to the everyday behavior of Complex Dynamic Systems?

Indeed, there is a good and large number of situations or problems where today's models and solutions fail to give satisfactory answers. Where have we failed? I believe that our weakness lies in:

- a) Our inability to comprehend and understand well and precisely the actual dynamic and chaotic behavior of CDS in the presence of uncertainty, fuzziness and structural complexity and

- b) due to uncertainty, fuzziness and structural complexity we have different interpretations and mathematical explanations by different people for the same real physical system.

Are we happy? Certainly not. Omnipresent in realistic settings, uncertainty and fuzziness constitutes a major obstacle to modeling and effectively analyzing Complex Dynamic Systems. How do we utilize knowledge and learning? Do we need new advance, innovative approaches? There appears to be a lack of new ideas in driver behavior modeling. Although behavioral research is under some pressure, it seems too facile to attribute this deplorable state of affairs only to a lack of research funds.

In my opinion the causal chain may well run in the opposite direction. An analysis of what is wrong has led me to the conclusion that human factors research in the area of driver behavior has hardly been touched by the 'cognitive revolution' that swept psychology in the past fifteen years. A more cognitive approach might seem advisable and the 'promise of progress' of such an approach should be assessed. Such an approach is provided with the advanced Fuzzy Cognitive Maps. This topic is addressed next.

\section{Modelling Using Fuzzy Cognitive Maps}

Fuzzy Cognitive Maps came as a combination of the methods of fuzzy logic and neural networks was first introduced by Kosko [16] only 30 years ago. A detailed presentation of FCM is provided in [18]. 
They constitute a computational method that is able to examine situations during which the human thinking process involves fuzzy or uncertain descriptions. An FCM presents a graphical representation used to describe the cause and effect relations between nodes, thus giving us the opportunity to describe the behavior of a system in a simple and symbolic way. In order to ensure the operation of the system.

FCMs embody the accumulated knowledge and experience from experts who know how the system behaves in different circumstances. This knowledge is extracted using linguistic variables which then are transformed to numeric values using a defuzzification method. In other words, they recommend a modeling process consisting of an array of interconnected and interdependent nodes $C_{i}$ (variables), as well as the relationships between them $\mathrm{W}$ (weights). Concepts take values in the interval $[0,1]$ and weights belong in the interval $[-1,1]$. Fig.2 shows a representative diagram of a FCM.

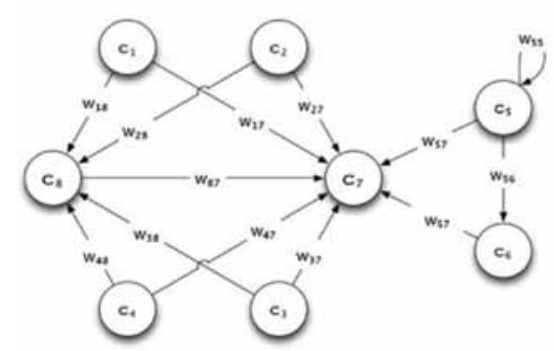

Figure 2: Fuzzy Cognitive Map.

The full procedure of the development of a FCM follows the below steps:

- $\quad$ Step 1: Experts select the number and the kind of concepts $C_{i}$ that constitute the Fuzzy Cognitive Map

- Step 2: Each expert defines the relationship between the concepts

- Step 3: They define the kind and the value of the relationship between the two nodes

- Step 4: Experts describe the existing relationship firstly as 'negative' or 'positive' and secondly, as a degree of influence using a linguistic variable, such as 'low', 'medium', 'high' etc.

The sign of each weight represents the type of influence between concepts. There are three types of interconnections between two concepts $C_{i}$ and $C_{j}$ :

- $\quad w_{i j}>0$, an increase or decrease in $C_{i}$ causes the same result in concept $C_{j}$.
- $\quad w_{i j}<0$, an increase or decrease in $C_{i}$ causes the opposite result in $C_{j}$.

- $w_{i j}=0$, there is no interaction between concepts $C_{i}$ and $C_{j}$.

The degree of influence between the two concepts is indicated by the absolute value of $w_{i j}$. During the simulation the value of each concept is calculated using the following rule:

$$
A_{i}(t)=f\left(k_{1} A_{i}(t-1)+k_{2} \sum_{\substack{j=1 \\ j \neq i}}^{n} A_{j}(t-1) w_{j i}\right)
$$

Where $\mathrm{t}$ represents time, $\mathrm{n}$ is the number of concepts and $\mathrm{f}$ is the sigmoid function given by the following equation:

$$
f=\frac{1}{1+e^{-\lambda x}}
$$

Where $\lambda>0$ determines the steepness of function $f$. The FCM's concepts are given some initial values which are then changed depending on the weights; the way the concepts affect each other. The calculations stop when a steady state is achieved, the concepts' values become stable. A more comprehensive mathematical presentation of FCMs with application to real problems with very useful results is provided in [17],[19]-[29].

There have been many efforts for the evolution of FCM over the last few years, but Simulation software tools are still missing.

\section{Drawbacks of Fuzzy Cognitive Maps}

In the previous sections trying to answer all challenging problems and questions in modelling and simulating CDS, FCMs were proposed as a new alternative and innovative approach to deal with these fundamental issues. The mathematical presentation along with the two examples from real problems give the academic and scientific communities hopes for overcoming some of the problems been encountered in modelling and simulating CDS using the classical approaches

However, with the current modeling of FCMs and their extensive use in solving many real problems and applications, various interesting and challenging problems and drawbacks have emerged. The early hope and enthusiasm that FCM would be a strong and effective approach to be able to solve the difficult problems of the Complex Dynamic Systems. However, FCMs theories are around only less than 30 years. 
We should not give up now. We must re-address the basic fundamentals that drove the scientific and academic community to develop FCMs, while at the same time keeping the core of the 'initial philosophy and methodology' intact. Artificial Intelligence (AI) has been around more than 70 years and despite the skepticism of some well known scientists AI still continues strong on funding and research efforts.

Prof Stephen Hawking, one of Britain's pre-eminent scientists, has said that efforts to create thinking machines pose a threat to our very existence. He told in an interview the BBC: 'The development of full artificial intelligence could spell the end of the human race.' His warning came in response to a question about a revamp of the technology he uses to communicate, which involves a basic form of AI. The theoretical physicist, who has the motor neurone disease amyotrophic lateral sclerosis (ALS), is using a new system developed by Intel to speak using AI. Prof Hawking is not alone in fearing for the future. In the short term, there are concerns that clever machines capable of undertaking tasks done by humans until now will swiftly destroy millions of jobs.

In the longer term, the technology entrepreneur Elon Musk has warned that AI is "our biggest existential threat". Many believe that AI is our biggest scientific mistake especially if it is left to scientists and politicians that do not have the human beeing as the center of our activities and/or they do not respect human values.

Therefore re-addressing the FCMs we should be careful and wise. Let us restate what is a FCM and why we claim that is a promising and innovative method for studying CDS

A Fuzzy Cognitive Map (FCM) provided a way to identify the most important structural elements in modeling and controlling Complex Dynamic Systems (CDS). Complete, efficient and practical mechanisms to analyze and predict the evolution of data been fuzzy, incomplete, and vague in CDS were not available for years due to several reasons. Numerical data for years have been considered as crisp and exact values.

However today most data may be fuzzy, uncertain or hard to come by, and the formulation of a precise mathematical model may be difficult, costly or even impossible. Then efforts to introduce knowledge on these systems should rely on natural language arguments and the human intervention in the absence of formal models.

However, although very efficient and simple to use, FCM are causal maps (a subset of cognitive maps that only allow basic symmetric and monotonic causal relations between concepts), and, in most applications, avoiding the need to use extensive and time consuming differential equation models, while obtaining very interesting and encouraging results. By using true qualitative modelling techniques, FCM obtained results that look more realistic (plausible) than those obtained using quantitative approaches - where results almost never show the short term uncertainties that are so characteristic of qualitative real-world dynamic systems. In the end, the results of the FCM model and all related applications, that were developed more than 25 years ago, are surprisingly realistic and could have been used to predict and avoid the current world economic crisis, even if one considers its necessary incompleteness.

An FCM is a qualitative mathematical tool rather than a quantitative tool. It provides a simple, flexible and straightforward approach to model the dynamic behavior of a complex system, which is composed of various components or subsystems. An FCM can always describe any CDS using a mathematical model with the following six (6) characteristics or attributes:

1) Defined causality indicating positive or negative relationship between all components

2) The causal links are always dynamic and never static

3) Past knowledge of the CDS dynamic behavior is available and reliable

4) Human-like reasoning and

5) Always availability of experts knowing the dynamic behavior of the CDS.

Given that the above hold and the FCM methodologies, so far been developed, we can model any given CDS. Solutions to this drawback were also provided there. There also other drawbacks of FCM that need to be addressed and been solved. These solutions are going to overcome some of these limitations and offer more accurate results and a better view and knowledge of the CDS.

One major drawback that has been raised by the author of this article is that concepts of an FCM include everything: states, inputs, outputs, constraints and all other parameters which are going to be examined regardless their nature [29]. However this is not mathematically correct and logical in any scientific approach. Why, for example in classic FCM theories the fact that some concepts are not being affected by others thus they have to stay static through the whole iteration process. 
However due to the current approach, Eq.1 and Eq.2 their value changes after the first iteration which is not correct. In addition having all variables in one 'concept vector' the iteration step $\mathrm{k}$ in Eq.1, is the same for all concepts which is also not true in real problems and mathematically not correct. Why must the inputs and outputs of the CDS change at every iteration step $\mathrm{k}$ ?

For example, on a health treatment of a patient, why must the inputs (concepts) (e.g. the medication dose of a drug that is given every morning) and the outputs (e.g. the blood test results (concepts) that are monitored every two or three days) be changed every time the 'health conditions' (concepts) of the patient is monitored every second or every hour? However, this is the case using classic FCM theories especially using equations 1 and 2.

Even the calculation method of the values of the concepts, (Eq.1) has a serious drawback. The calculation equation takes into consideration the change that each concept cause separately instead of the total change which is caused to the concept $C_{i}$. This results in a large increase to the value of the concept $C_{i}$ that goes far beyond the interval $[0,1]$. This is the reason why the sigmoid function (Eq.2) is needed; to suppress the result to the interval $[0,1]$.

However due to the shape of the sigmoid curve any concept value beyond 3 leads the sigmoid function to correspond it to the value 1 which is greatly problematic as the final output is corresponded to the linguistic variable 'high' even if this is not always the expected or correct result. However continuing on the subject of the sigmoid function, there is another drawback that leads to high output values. This is the fact that the center of the sigmoid curve instead of being on the $(0,0)$ point on the $x y$ axis it is on the $(0.5,0)$ point. This means that each concept's lowest value can be the 0.5 . This problem combined with the first one makes it difficult to interpret the obtained results.

Continuing with the NHL learning method, while running several simulations we have observed that due to the way weights are being calculated if the number of iterations of the algorithm is increased, in order to reach a steady state, the causality reverses and all or some of the $w_{i j}$ become positive. This is a very serious drawback as it changes the causality between concepts and in several occasions instead of having a lower we are going to have a larger result which can cause serious problems not only in the interpretation of the obtained results but also on stability issues to a number of systems.
There are a number of other drawbacks that need to be addressed but this should be the work of future papers. However for the above raised drawbacks some solutions and explanations must be given. The research team of the Laboratory for Automation and Robotics under the supervision of the author of this paper have provided some interesting and valuable solutions.

As it was mentioned, above in this section, in the classic FCM representation ALL the concepts are ALL the parameters which are going to be examined regardless their nature. However, in a CDS, even when it is described in a fuzzy way through an FCM the main concept is the same.

Each system has its states, inputs, outputs and other parameters and constraints. However, since an FCM is a representation of such a system, this fundamental characteristic should be taken into consideration. For this reason, as in the classic control theory methods [2]-[4], [7][8], the concepts of a Fuzzy Cognitive Maps are separated into the following three categories:

A) Fuzzy State Concepts: The concepts describing the dynamic operation of the system, $\mathrm{x}$.

B) Fuzzy Input Concepts: The inputs of the system, $u$ and

C) Fuzzy Output Concepts: The concepts describing the outputs of the system, $y$

In this way a better knowledge of the dynamic behavior of the CDS is gained. The proposed separation facilitates not only the understanding of the system's operation but also the calculation of the concepts' values in their physical nature as the states, inputs and outputs of the real system.

Authors have proposed a new calculation rule. The two equations extracted from the classic FCM are the followings:

$$
\begin{aligned}
x_{k+1} & =f\left[A x_{k}+B u_{k}\right] \\
y_{k} & =f\left[C x_{k}+D u_{k}\right]
\end{aligned}
$$

where $x_{k} \in \mathrm{R}^{\mathrm{n}}$ is a state vector, $u_{k} \in \mathrm{R}^{\mathrm{r}}$ is an exogenous known input vector, $y_{k} \in \mathrm{R}^{\mathrm{m}}$ is the output vector and $f$ is an activation function. The new model was implemented for first time in diagnosing meniscus injury in the IFAC World Congress 2017 with very encouraging results [29]. 


\section{Conclusions and Future Research}

In this overview paper one of the most difficult and challenging problems in modelling, analyzing and simulating Complex Dynamic Systems (CDS) has been seriously addressed. The analysis and efficient control of CDS are impossible without a formal model of the system. However, today's technologies for building such models for CDS are not sufficient. Then the necessary simulations cannot describe the dynamic behavior of the system under study. Qualitative description of most of the parameters of Complex Dynamic Systems results inevitably in fuzziness, complexity and uncertainty.

Thus the human cognition and presence is absent from the modelling band simulation approaches. New approaches are needed. Fuzzy Cognitive Maps seem to provide a possible solution. However, they do have quite a few drawbacks that must be resolved.

\section{Challenging future research directions? Just take} each question been raised in this paper and you have a big number of research challenges:

- new models of FCMs for CDS using learning methods;

- develop new simulation models and software tools using intelligent systems and advanced neural network theories;

- develop mathematical models using new advance FCMs for different applications and using a number of experts;

- develop new software tools for various CDS and perform extensive simulations, and

- develop a new Intelligent and Cognitive Sytems Control theory using the human as the center of such an effort [30].

\section{References}

[1] Luenberger D G. Introduction to dynamic systems: theory, models, and applications. Wiley, 1979.

[2] Beltrami E J. Mathematics for Dynamic Modeling, Vol 2. Academic Press, 1998

[3] Sandquist G M.Introduction to System Science.Prentice Hall, Inc. Englewood. Cliffs, N.J. 1985.

[4] Bar-Yam Y. Dynamics of Complex Systems. Reading, MA: Addison-Wesley, 1997.
[5] Mesarovic M, Macko D, Takahara Y. Theory of hierarchical multilevel systems. New York: Academic Press, 1970.

[6] Levin S A. Complex adaptive systems: Exploring the known, the unknown and the unknowable. Bulletin of the American Mathematical Society. 2003; 40:3-19.

[7] Nelson R J. Structure of Complex Systems. Philosophy of Science Association. 1976; 2:523-542.

[8] Ottino J M. Engineering complex systems. Nature, 2004; 427: 399-399.

[9] Banks J (editor). Handbook of Simulation: Principles, Methodology, Advances, Applications, and Practice. (1st Edition), Engineering and Management Press (EMP), 2001

[10] Pooch U D, Wall J A. Discrete Event Simulation: A Practical Approach. Cre Press Computer Engineering Series, Mishawaka, IN, U.S.A, 1993.

[11] Hannon R E. Systems simulation: the art and science. Prentice-Hall, 1975.

[12] Widman L E, Loparo K A, Nielsen N R. Artificial Intelligence, Simulation and Modeling. Wilye Interscience, N.Y. 1989.

[13] Law A. Simulation Modeling and Analysis , McGraw-Hill Series in Industrial Engineering and Management. 5th Edition, 2013.

[14] Thesen A, Travis L. E. Simulation for Decision Making. West Group,1992.

[15] Ragazzini J R, et al. Analysis of problems in dynamics by electronic circuits. Proceedings of the IRE, vol. 35. p. 444, May 1947.

[16] Kosko B. Fuzzy cognitive maps. International Journal of man-machine studies. 1986; 24(1):65-75.

[17] Groumpos P P, Stylios C D. Modeling supervisory control systems using fuzzy cognitive maps. Chaos Solitons \& Fractals. 2000; 113: 329-336.

[18] Groumpos P P. Fuzzy Cognitive Maps: Basic Theories and their Application to Complex Systems. Glykas M. (ed) Fuzzy Cognitive Maps: Advances in Theory, Methodologies, Tools and Applications. Springer-Verlag Berlin, Heidelberg, 2010; 247: 1-22.

[19] Papageorgiou E I, Stylios C D, Groumpos P P. Active Hebbian learning algorithm to train fuzzy cognitive maps. International Journal of Approximate Reasoning, 2004;37: 219-249.

[20] Groumpos P P, Anninou A P. A theoretical mathematical modeling of Parkinson's disease using Fuzzy Cognitive Maps. 12th International Conference on Bioinformatics and Bioengineering (BIBE 2012), pp 677 - 682.

[21] Taber W R. Knowledge processing with fuzzy cognitive maps. Expert Systems with Applications, 1991; 2(1): 83-87. 
[22] Lee S, Ahn H. Fuzzy cognitive map based on structural equation modeling for the design of controls in businessto-consumer e-commerce web-based systems. Expert Systems with Applications. 2009; 36(7): 10447-10460

[23] Stylios C D, Groumpos P P. Modeling complex systems using fuzzy cognitive maps. IEEE Transactions on Systems, Man and Cybernetics, Part A: Systems and Humans. 2004; 34(1):155-162.

[24] Carmona M A, Barbancho J, Larios D F, León C. Applying case based reasoning for prioritizing areas of business management. Expert Systems with Applications. 2013; 40(9):3450-3458.

[25] Carmona M A, Barbancho J, Larios D F, León C. Applying case based reasoning for prioritizing areas of business management. II, Expert Systems with Applications. 2013; 40(9):3450-3458.

[26] Anninou A P, Groumpos P P, Polychronopoulos P. Modeling Health Diseases Using Competitive Fuzzy Cognitive Maps. Artificial Intelligence Applications and Innovations. Springer Berlin Heidelberg, 2013, pp.88-95
[27] Bourgani E, Stylios C D, Manis G, Georgopoulos V C. Time dependent fuzzy cognitive maps for medical diagnosis. In Hellenic Conf. on AI, 544-554, Springer, 2014.

[28] Carvalho J P, Tome J. Issues on the stability of fuzzy cognitive maps and rule-based fuzzy cognitive maps. In Proceedings of the 21st International Conference of the North American Fuzzy Information Processing Society, NAFIPS2002, New Orleans, 2002.

[29] Anninou A. P, Groumpos P P, Poulios P, Gkliatis I. A New Approach of Dynamic Fuzzy Cognitive Knowledge Networks in Modelling Diagnosing Process of Meniscus Injury”. IFAC-PapesOnLine, 2017, 50(1), 5861-5866.

[30] Groumpos P P. Intelligent Control and Cognitive Control: Issues and Challenges. In Brain Function Assessment in Learning, First Intern. Conf. BFAL, Proc. by Springer pp. 1-3, Patras, Greece, September 2017. 


\section{ASIM - Buchreihen / ASIM Book Series}
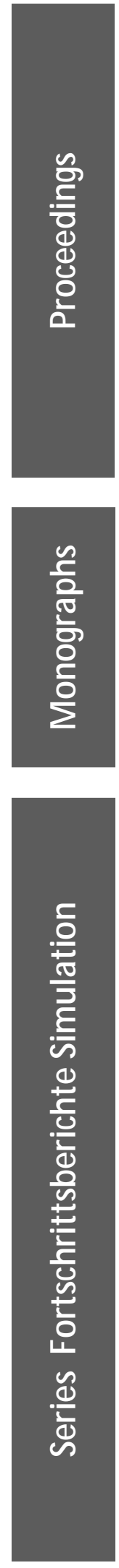

Simulation in Production and Logistics 2017 - 17. ASIM Fachtagung Simulation in Produktion und Logistik Sigrid Wenzel, Tim Peter (Hrsg.); kassel university press GmbH, Kassel, 2017; ISBN 978-3-7376-0192-4 (print), ISBN 978-3-7376-0193-1 (online); ASIM M itteilung AM 164.

Simulation in Production und Logistics 2015 - 16. ASIM-Fachtagung Simulation in Produktion und Logistik M. Raabe, U. Clausen (Hrsg.); ISBN 978-3-8396-0936-1, Stuttgart: Fraunhofer Verlag, 2015.

Simulation in Produktion und Logistik 2013: Entscheidungsunterstützung von der Planung bis zur Steuerung W. Dangelmaier, C. Laroque, A. Klaas (Hrsg.); ISBN 978-3-942647-35-9, HNI-Verlagsschriftenreihe, Heinz Nixdorf Institut, Paderborn, 2013.

Modellierung, Regelung und Simulation in Automotive und Prozessautomation - Proc. 5. ASIM-Workshop Wismar 2011. C. Deatcu, P. Dünow, T. Pawletta, S. Pawletta (eds.), ISBN 978-3-901608-36-0, ASIM /ARGESIM, Wien, 2011.

Simulation in Produktion und Logistik 2010: Integrationsaspekte der Simulation - Technik, Organisation und Personal. G. Zülch, P. Stock, (Hrsg.), ISBN 978-3-86644-558-1, KIT Scientific Publ. Karlsruhe, 2010

Simulation und Optimierung in Produktion und Logistik - Praxisorientierter Leitfaden mit Fallbeispielen. L. März, W. Krug, O. Rose, G. Weigert , G. (Hrsg.); ISBN 978-3-642-14535-3, Springer, 2011.

Verifikation und Validierung für die Simulation in Produktion und Logistik - Vorgehensmodelle und Techniken. M. Rabe, S. Spieckermann, S. Wenzel (eds.); ISBN: 978-3-540-35281-5, Springer, Berlin, 2008.

Qualitätskriterien für die Simulation in Produktion und Logistik - Planung und Durchführung von Simulationsstudien. S. Wenzel, M. Weiß, S. Collisi - Böhmer, H. Pitsch, O. Rose (Hrsg.); ISBN: 978-3-540-35281-5, Springer, Berlin, 2008.

Patrick Einzinger: A Comparative Analysis of System Dynamics and Agent-Based Modelling for Health Care Reimbursement Systems. FBS 24; ISBN 978-3-901608-75-9 (online), ASIM / ARGESIM Vienna, 2014; www.tuverlag.at - print-on-demand.

M artin Bruckner: Agentenbasierte Simulation von Personenströmen mit unterschiedlichen Charakteristiken. FBS 23; ISBN 978-3-901608-74-2 (online), ASIM/ARGESIM Vienna, 2014; www.tuverlag.at - print-on-demand

Stefan Emrich: Deployment of Mathematical Simulation Models for Space Management. FBS 22; ISBN 978-3-901608-73-5 (online), ASIM/ARGESIM Vienna, 2013; www.tuverlag.at - print-on-demand

Xenia Descovich: Lattice Boltzmann Modeling and Simulation of Incompressible Flows in Distensible Tubes for Applications in Hemodynamics. FBS 21; ISBN 978-3-901608-71-1 (online), ASIM /ARGESIM Vienna, 2012; www.tuverlag.at - print-on-demand

Florian M iksch: Mathematical Modeling for New Insights into Epidemics by Herd Immunity and Serotype Shift. FBS 20, ISBN 978-3-901608-70-4 (online), ASIM / ARGESIM Vienna, 2012; www.tuverlag.at - print-on-demand

Shabnam Tauböck: Integration of Agent Based M odelling in DEVS for Utilisation Analysis: The M oreSpace Project at TU Vienna. FBS 19; ISBN 978-3-901608-69-8 (online), ASIM/ARGESIM Vienna, 2012; www.tuverlag.at - print-on-demand

Christian Steinbrecher: Ein Beitrag zur prädiktiven Regelung verbrennungsmotorischer Prozesse. FBS 18; ISBN 978-3-901608-68-1 (print), 978-3-901608-72-8 (online), ASIM / ARGESIM Vienna, 2010.

Olaf Hagendorf: Simulation-based Parameter and Structure Optimisation of Discrete Event Systems FBS 17; ISBN 978-3-901608-67-4 (print), 978-3-901608-99-5 (online), ASIM / ARGESIM Vienna, 2010. 


\section{SNE Simulation News \\ EUROSIM Data and Quick Info}
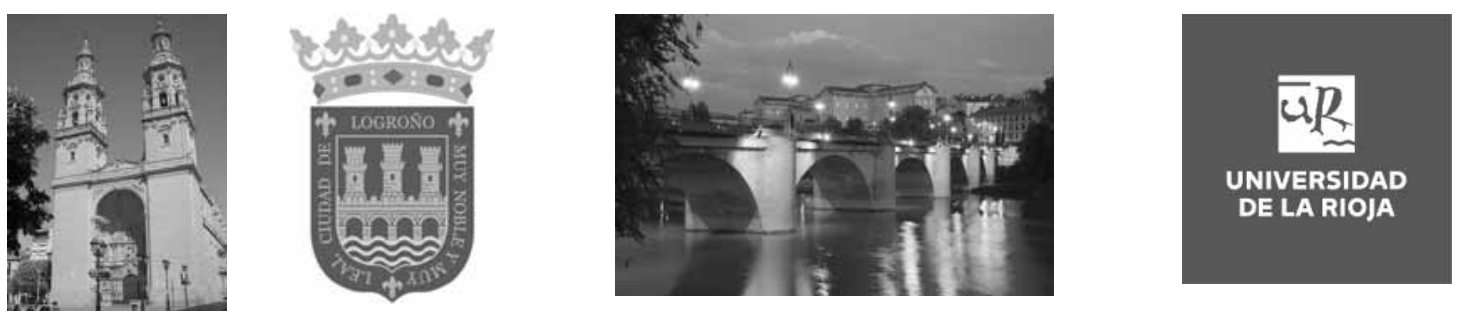

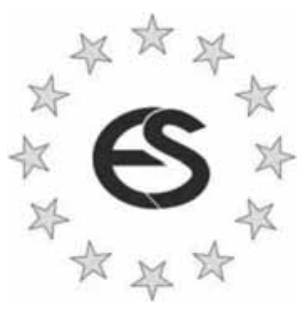

Contents

Short Info EUROSIM N2

Short Info ASIM, CEA-SM SG ..N3

Short Info CROSSIM , CSSS, DBSS, FRANCOSIM ...........N4

Short Info HSS, ISCS, LIOPHANT, LSS ............................N5

Short Info KA-SIM , PSCS, SIM S .................................... 6

Short Info SLOSIM , RNSS, UKSIM ................................N7

Short Info ROM SIM , M IM OS, Albanian Soc. ...............N8

NewS EUROSIM - Conferences .................................N9

News EUROSIM - New EUROSIM Website ................N10

News EUROSIM - New SNE Website ........................N12

News ASIM / CEA-SM SG Conferences .....................N13

Simulation Notes Europe SNE is the official membership journal of EUROSIM and distributed / available to members of the EUROSIM Societies as part of the membership benefits.

If you have any information, announcement, etc. you want to see published, please contact a member of the editorial board in your country or the editorial office. For scientific publications, please contact the EiC.

This EUROSIM Data \& Quick Info compiles data from EUROSIM societies and groups: addresses, weblinks, and officers of societies with function and email, to be published regularly in SNE issues. This information is also published at EUROSIM's website www.eurosim.info.

\section{SNE Reports Editorial Board}

EUROSIM Emilio Jiménez, emilio.jimenez@unirioja.es Andreas Körner, andreas.koerner@tuwien.ac.at Miguel Mujica Mota, m.mujica.mota@hva.nl

ASIM A. Körner, andreas.koerner@tuwien.ac.at CEA-SMSG Emilio Jiménez, emilio.jimenez@unirioja.es CROSSIM Vesna Dušak,vdusak@foi.hr CSSS Mikuláš Alexík, alexik@frtk.utc.sk DBSS M. Mujica Mota, m.mujica.mota@hva.nl FRANCOSIM Karim Djouani, djouani@u-pec.fr HSS András Jávor, javor@eik.bme.hu ISCS M. Savastano, mario.savastano@unina.it LIOPHANT F. Longo, f.longo@unical.it LSS Yuri Merkuryev,merkur@itl.rtu.lv PSCS Zenon Sosnowski, zenon@ii.pb.bialystok.pl RNSS Y. Senichenkov, senyb@dcn.icc.spbstu.ru SIMS Esko Juuso, esko.juuso@oulu.fi SLOSIMVito Logar,vito.logar@fe.uni-lj.si UKSIM A. Orsoni, A.Orsoni@kingston.ac.uk KA-SIM Edmond Hajrizi, info@ka-sim.com MIM OS Paolo Proietti, roma@mimos.it ROMSIM Marius Radulescu, mradulescu@ici.ro Albanian Society Kozeta Sevrani, kozeta.sevrani@unitir.edu.al SNE Editorial Office / ARGESIM

$\rightarrow$ www.sne-journal.org, www.eurosim.info 䤵office@sne-journal.org, Andreas Körner, (info, news) 棒eic@sne-journal.org, Felix Breitenecker (publications) $\triangle$ SNE Editorial Office, Andreas Körner c/ o ARGESIM / M athematical M odelling $\&$ Simulation Group, TU Wien /101, Wiedner Hauptstrasse 8-10, 1040 Vienna , Austria 


\section{EUROSIM}

\section{$\boldsymbol{S}$ * Federation of European Simulation Societies}

General Information. EUROSIM, the Federation of European Simulation Societies, was set up in 1989. The purpose of EUROSIM is to provide a European forum for simulation societies and groups to promote advancement of modelling and simulation in industry, research, and development. $\rightarrow$ www.eurosim.info

Member Societies. EUROSIM members may be national simulation societies and regional or international societies and groups dealing with modelling and simulation. At present EUROSIM has 16 Full Members and 2 Observer Members, and one member candidate.

\begin{tabular}{ll}
\hline ASIM & $\begin{array}{l}\text { Arbeitsgemeinschaft Simulation } \\
\text { Austria, Germany, Switzerland }\end{array}$ \\
\hline CEA-SM SG & $\begin{array}{l}\text { Spanish M odelling and Simulation Group } \\
\text { Spain }\end{array}$ \\
\hline CROSSIM & $\begin{array}{l}\text { Croatian Society for Simulation M odeling } \\
\text { Croatia }\end{array}$ \\
\hline CSSS & $\begin{array}{l}\text { Czech and Slovak Simulation Society } \\
\text { Czech Republic, Slovak Republic }\end{array}$ \\
\hline DBSS & $\begin{array}{l}\text { Dutch Benelux Simulation Society } \\
\text { Belgium, Netherlands }\end{array}$ \\
\hline FrANCOSIM & $\begin{array}{l}\text { Société Francophone de Simulation } \\
\text { Belgium, France }\end{array}$ \\
\hline HSS & Hungarian Simulation Society; Hungary \\
\hline ISCS & $\begin{array}{l}\text { Italian Society for Computer Simulation } \\
\text { Italy }\end{array}$ \\
\hline KA-SIM & Kosovo Simulation Society, Kosovo \\
\hline LOPHANT & $\begin{array}{l}\text { LIOPHANT Simulation Club } \\
\text { Italy \& International }\end{array}$ \\
\hline LSS & Latvian Simulation Society; Latvia \\
\hline PSCS & $\begin{array}{l}\text { Polish Society for Computer Simulation } \\
\text { Poland }\end{array}$ \\
\hline MIM OS & $\begin{array}{l}\text { Italian M odelling and Simulation } \\
\text { Association, Italy, Observer M ember }\end{array}$ \\
\hline RNSS & $\begin{array}{l}\text { Russian National Simulation Society } \\
\text { Russian Federation }\end{array}$ \\
\hline ROM SIM & $\begin{array}{l}\text { Romanian Society for Modelling and Sim- } \\
\text { ulation, Romania, Observer M ember }\end{array}$ \\
\hline SIMS & $\begin{array}{l}\text { Simulation Society of Scandinavia } \\
\text { Denmark, Finland, Norway, Sweden }\end{array}$ \\
\hline SLOSIM & $\begin{array}{l}\text { Slovenian Simulation Society } \\
\text { Slovenia }\end{array}$ \\
\hline UKSIM & $\begin{array}{l}\text { United Kingdom Simulation Society } \\
\text { UK, Ireland }\end{array}$ \\
\hline
\end{tabular}

EUROSIM Board / Officers. EUROSIM is governed by a board consisting of one representative of each member society, president and past president, and representatives for SNE Simulation Notes Europe. The President is nominated by the society organising the next EUROSIM Congress. Secretary, Secretary to the Board, and Treasurer are elected out of members of the board.

\begin{tabular}{ll}
\hline President & $\begin{array}{l}\text { Emilio Jiménez (CAE-SM SG), } \\
\text { emilio.jimenez@ unirioja.es }\end{array}$ \\
\hline Past President & $\begin{array}{l}\text { Esko Juuso (SIM S) } \\
\text { esko.juuso@ oulu.fi }\end{array}$ \\
\hline Secretary & $\begin{array}{l}\text { M. Mujica M ota (DBSS), } \\
\text { m.mujica.mota@ hva.nl }\end{array}$ \\
\hline Treasurer & $\begin{array}{l}\text { Felix Breitenecker (ASIM) } \\
\text { felix.breitenecker@ tuwien.ac.at }\end{array}$ \\
\hline $\begin{array}{c}\text { Secretary to the } \\
\text { Board }\end{array}$ & $\begin{array}{l}\text { Andreas Körner } \\
\text { andreas.koerner@tuwien.ac.at }\end{array}$ \\
\hline SNE & Felix Breitenecker \\
Representative & felix.breitenecker@tuwien.ac.at \\
\hline
\end{tabular}

SNE - Simulation Notes Europe. SNE is a scientific journal with reviewed contributions as well as a membership newsletter for EUROSIM with information from the societies in the News Section. EUROSIM societies are offered to distribute to their members the journal SNE as official membership journal. SNE Publishers are EUROSIM, ARGESIM and ASIM.

\begin{tabular}{|c|c|}
\hline $\begin{array}{l}\text { SNE } \\
\text { Editor-in-Chief }\end{array}$ & $\begin{array}{l}\text { Felix Breitenecker } \\
\text { felix.breitenecker@tuwien.ac.at }\end{array}$ \\
\hline
\end{tabular}

EUROSIM Congress. EUROSIM is running the triennial conference series EUROSIM Congress. The congress is organised by one of the EUROSIM societies.

EUROSIM 2019, the $10^{\text {th }}$ EUROSIM Congress, will be organised by CAE-SMSG, the Spanish simulation society, in La Rioja, Logroño, Spain, in July 2019.

\begin{tabular}{l}
\hline Chairs / Team EUROSIM 2019 \\
\hline $\begin{array}{c}\text { Emilio Jiménez, EUROSIM President, } \\
\text { emilio.jimenez@unirioja.es }\end{array}$ \\
$\begin{array}{c}\text { Juan Ignacio Latorre, juanignacio.latorre@ unavarra.es } \\
\rightarrow \text { www.eurosim.info }\end{array}$
\end{tabular}




\section{EUROSIM Member Societies}

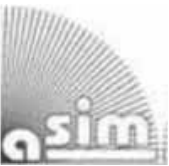

\section{ASIM \\ German Simulation Society \\ Arbeitsgemeinschaft Simulation}

ASIM (Arbeitsgemeinschaft Simulation) is the association for simulation in the German speaking area, servicing mainly Germany, Switzerland and Austria. ASIM was founded in 1981 and has now about 600 individual members, and 90 institutional or industrial members.

$\rightarrow$ www.asim-gi.org with members' area

埄info@asim-gi.org,admin@asim-gi.org

$\bowtie$ ASIM - Inst. f. Analysis and Scientific Computing

Vienna University of Technology (TU Wien)

Wiedner Hauptstraße 8-10, 1040 Vienna, Austria

\begin{tabular}{|c|c|}
\hline \multicolumn{2}{|l|}{ ASIM Officers } \\
\hline President & $\begin{array}{l}\text { Felix Breitenecker } \\
\text { felix.breitenecker@tuwien.ac.at }\end{array}$ \\
\hline \multirow[t]{2}{*}{ Vice presidents } & Sigrid Wenzel, s.wenzel@uni-kassel.de \\
\hline & $\begin{array}{l}\text { T. Pawletta, } \\
\text { thorsten.pawletta@hs-wismar.de }\end{array}$ \\
\hline Secretary & $\begin{array}{l}\text { Ch. Deatcu, christina.deatcu@hs-wismar.de } \\
\text { A. Körner, andreas.koerner@tuwien.ac.at }\end{array}$ \\
\hline Treasurer & Anna M athe, anna.mathe@tuwien.ac.at \\
\hline \multirow{3}{*}{$\begin{array}{l}\text { Membership } \\
\text { Affairs }\end{array}$} & S. Wenzel, s.wenzel@uni-kassel.de \\
\hline & Ch. Deatcu, christina.deatcu@ hs-wismar.de \\
\hline & F. Breitenecker, felix.breitenecker@tuwien.ac.at \\
\hline \multirow[t]{2}{*}{ Repr. EURoSIm } & F. Breitenecker, felix.breitenecker@tuwien.ac.at \\
\hline & A. Körner, andreas.koerner@tuwien.ac.at \\
\hline \multirow{2}{*}{$\begin{array}{l}\text { Int. Affairs - } \\
\text { GI Contact }\end{array}$} & N. Popper, niki.popper@drahtwarenhandlung.at \\
\hline & O. Rose, Oliver.Rose@tu-dresden.de \\
\hline \multirow[t]{2}{*}{$\begin{array}{l}\text { Editorial Board } \\
\text { SNE }\end{array}$} & $\begin{array}{l}\text { T. Pawletta, } \\
\text { thorsten.pawletta@hs-wismar.de }\end{array}$ \\
\hline & Ch. Deatcu, christina.deatcu@ @s-wismar.de \\
\hline Web EuRoSIM & A. Körner, andreas.koerner@tuwien.ac.at \\
\hline
\end{tabular}

ASIM is organising / co-organising the following international conferences:

- ASIM Int. Conference 'Simulation in Production and Logistics' - bi-annual

- ASIM 'Symposium Simulation Technique'

- biannual

- MATHMOD Int. Vienna Conference on Mathmatical Modelling - triennial

Furthermore, ASIM is co-sponsor of WSC - Winter Simulation Conference

\begin{tabular}{cl}
\hline ASIM Working Committee \\
\hline GM MS & $\begin{array}{l}\text { Methods in M odelling and Simulation } \\
\text { Th. Pawletta, thorsten.pawletta@hs-wismar.de }\end{array}$ \\
\hline SUG & $\begin{array}{l}\text { Simulation in Environmental Systems } \\
\text { J.Wittmann, wittmann@ informatik.uni-ham- } \\
\text { burg.de }\end{array}$ \\
\hline STS & $\begin{array}{l}\text { Simulation of Technical Systems } \\
\text { Walter Comerell, Commerell@ hs-ulm.de }\end{array}$ \\
\hline SPL & $\begin{array}{l}\text { Simulation in Production and Logistics } \\
\text { Sigrid Wenzel, s.wenzel@ uni-kassel.de }\end{array}$ \\
\hline EDU & $\begin{array}{l}\text { Simulation in Education/Education in Simulation } \\
\text { A. Körner, andreas.koerner@tuwien.ac.at }\end{array}$ \\
\hline BIG DATA & $\begin{array}{l}\text { Working Group Data-driven Simulation in Life } \\
\text { Sciences; niki.popper@ dwh.at }\end{array}$ \\
\hline & $\begin{array}{l}\text { Working Groups for Simulation in Business } \\
\text { Administration, in Traffic Systems, for Standardi- } \\
\text { sation, etc. }\end{array}$ \\
\hline
\end{tabular}

\section{CEA-SM SG - Spanish Modelling and Simulation Group}

CEA is the Spanish Society on Automation and Control and it is the national member of IFAC (International Federation of Automatic Control) in Spain. Since 1968 CEAIFAC looks after the development of the Automation in Spain, in its different issues: automatic control, robotics, SIMULATION, etc. The association is divided into national thematic groups, one of which is centered on Modeling, Simulation and Optimization, constituting the CEA Spanish Modeling and Simulation Group (CEA-SMSG). It looks after the development of the Modelling and Simulation (M\&S) in Spain, working basically on all the issues concerning the use of $M \& S$ techniques as essential engineering tools for decision-making and optimization.

$\rightarrow$ http://www.ceautomatica.es/grupos/

$\rightarrow$ emilio.jimenez@unirioja.es

simulacion@cea-ifac.es

$\triangle$ CEA-SMSG / Emilio Jiménez, Department of Electrical Engineering, University of La Rioja, San José de Calasanz 31, 26004 Logroño (La Rioja), SPAIN

\begin{tabular}{ll}
\hline \multicolumn{2}{l}{ CEA - SM SG Officers } \\
\hline President & $\begin{array}{l}\text { Emilio Jiménez, } \\
\text { emilio.jimenez@unirioja.es }\end{array}$ \\
\hline Vice president & $\begin{array}{l}\text { Juan Ignacio Latorre, } \\
\text { juanignacio.latorre@ unavarra.es }\end{array}$ \\
\hline Repr. EUROSIM & Emilio Jiménez, emilio.jimenez@ unirioja.es \\
\hline Edit. Board SNE & Emilio Jiménez, emilio.jimenez@ unirioja.es \\
\hline Web EuroSIM & Mercedes Perez mercedes.perez@ unirioja.es \\
\hline
\end{tabular}




\section{CROSSIM - Croatian Society for Simulation Modelling}

CROSSIM-Croatian Society for Simulation Modelling was founded in 1992 as a non-profit society with the goal to promote knowledge and use of simulation methods and techniques and development of education. CROSSIM is a full member of EUROSIM since 1997.

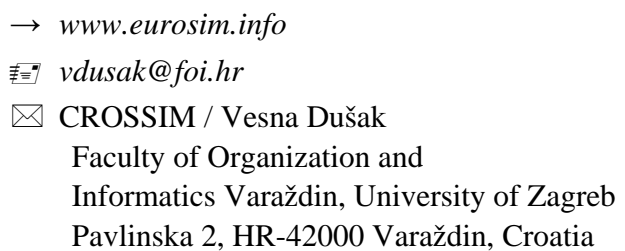

\begin{tabular}{ll}
\multicolumn{2}{l}{ CROSSIM Officers } \\
\hline President & Vesna Dušak, vdusak@foi.hr \\
\hline Vice president & Jadranka Božikov, jbozikov@ snz.hr \\
\hline Secretary & Vesna Bosilj-Vukšić, vbosilj@ efzg.hr \\
\hline Executive board & Vlatko Čerić, vceric@ efzg.hr \\
\cline { 2 - 2 } members & Tarzan Legović, legovic@ irb.hr \\
\hline Repr. EuRoSım & Jadranka Božikov, jbozikov@ snz.hr \\
\hline Edit. Board SNE & Vesna Dušak, vdusak@ foi.hr \\
\hline Web EuRoSım & Jadranka Bozikov, jbozikov@ snz.hr \\
\hline
\end{tabular}

\section{CSSS - Czech and Slovak CSSS Simulation Society}

CSSS -The Czech and Slovak Simulation Society has about 150 members working in Czech and Slovak national scientific and technical societies (Czech Society for Applied Cybernetics and Informatics, Slovak Society for Applied Cybernetics and Informatics). The main objectives of the society are: development of education and training in the field of modelling and simulation, organising professional workshops and conferences, disseminating information about modelling and simulation activities in Europe. Since 1992, CSSS is full member of EUROSIM.

$\rightarrow$ www.fit.vutbr.cz/CSSS

奉三” snorek@fel.cvut.cz

$\triangle$ CSSS / Miroslav Šnorek, CTU Prague

FEE, Dept. Computer Science and Engineering,

Karlovo nam. 13, 12135 Praha 2, Czech Republic

\begin{tabular}{ll}
\hline President & Miroslav Šnorek, snorek@ fel.cvut.cz \\
\hline Vice president & Mikuláš Alexík, alexik@ frtk.fri.utc.sk \\
\hline Scientific Secr. & A. Kavička, Antonin.Kavicka@upce.cz \\
\hline Repr. EuroSIM & Miroslav Šnorek, snorek@fel.cvut.cz \\
\hline Edit. Board SNE & Mikuláš Alexík, alexik@ frtk.fri.utc.sk \\
\hline Web EUROSIM & Petr Peringer, peringer@fit.vutbr.cz \\
\hline
\end{tabular}

\section{DBSS - Dutch Benelux Simulation Society}

The Dutch Benelux Simulation Society (DBSS) was founded in July 1986 in order to create an organisation of simulation professionals within the Dutch language area. DBSS has actively promoted creation of similar organisations in other language areas. DBSS is a member of EUROSIM and works in close cooperation with its members and with affiliated societies.

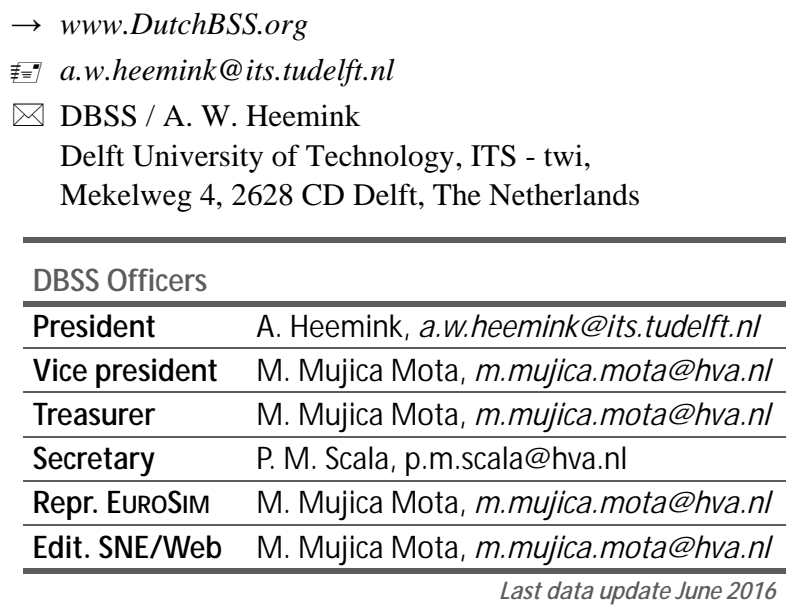

\section{FrANCOSIM - Société Francophone de Simulation}

FRANCOSIM was founded in 1991 and aims to the promotion of simulation and research, in industry and academic fields.

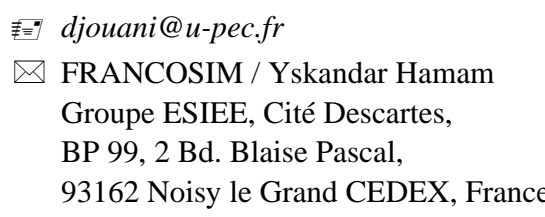

FRANCOSIM Officers

\begin{tabular}{ll}
\hline President & Karim Djouani, djouani@u-pec.fr \\
\hline Treasurer & François Rocaries, f.rocaries@esiee.fr \\
\hline Repr. EuRoSIM & Karim Djouani, djouani@u-pec.fr \\
\hline Edit. Board SNE & Karim Djouani, djouani@u-pec.fr \\
\hline
\end{tabular}




\section{HSS - Hungarian Simulation Society}

The Hungarian Member Society of EUROSIM was established in 1981 as an association promoting the exchange of information within the community of people involved in research, development, application and education of simulation in Hungary and also contributing to the enhancement of exchanging information between the Hungarian simulation community and the simulation communities abroad. HSS deals with the organization of lectures, exhibitions, demonstrations, and conferences.

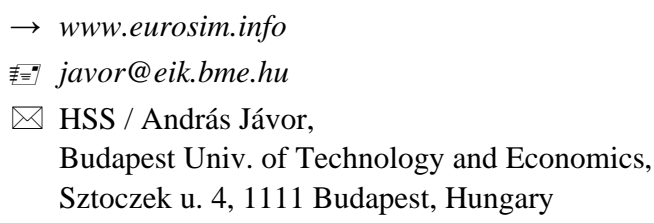

\begin{tabular}{ll} 
HSS Officers & \\
\hline President & András Jávor, javor@ eik.bme.hu \\
\hline Vice president & Gábor Szúcs, szucs@itm.bme.hu \\
\hline Secretary & Ágnes Vigh, vigh@itm.bme.hu \\
\hline Repr. EuRoSım & András Jávor, javor@ eik.bme.hu \\
\hline Deputy & Gábor Szúcs, szucs@itm.bme.hu \\
\hline Edit. Board SNE & András Jávor, javor@ eik.bme.hu \\
\hline Web EuRoSım & Gábor Szúcs, szucs@itm.bme.hu \\
\hline
\end{tabular}

\section{ISCS - Italian Society for Computer} Simulation

The Italian Society for Computer Simulation (ISCS) is a scientific non-profit association of members from industry, university, education and several public and research institutions with common interest in all fields of computer simulation.

$\rightarrow$ www.eurosim.info

邫Mario.savastano@uniina.it

$\triangle$ ISCS / Mario Savastano,

c/o CNR - IRSIP,

Via Claudio 21, 80125 Napoli, Italy

\begin{tabular}{ll}
\hline ISCS Officers & \\
\hline President & M. Savastano, mario.savastano@ unina.it \\
\hline Vice president & F. M aceri, Franco.M aceri@ uniroma2.it \\
\hline Repr. EuRoSIM & F. M aceri, Franco.M aceri@ uniroma2.it \\
\hline Secretary & $\begin{array}{l}\text { Paola Provenzano, } \\
\text { paola.provenzano@ uniroma2.it }\end{array}$ \\
\hline Edit. Board SNE & M. Savastano, mario.savastano@ unina.it \\
\hline
\end{tabular}

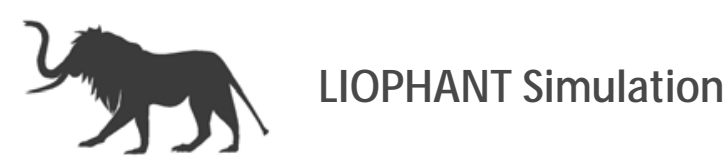

Liophant Simulation is a non-profit association born in order to be a trait-d'union among simulation developers and users; Liophant is devoted to promote and diffuse the simulation techniques and methodologies; the Association promotes exchange of students, sabbatical years, organization of International Conferences, courses and internships focused on M\&S applications.

$\rightarrow$ www.liophant.org

麦”;info@liophant.org

$\triangle$ LIOPHANT Simulation, c/o Agostino G. Bruzzone, DIME, University of Genoa, Savona Campus via Molinero 1, 17100 Savona (SV), Italy

\begin{tabular}{ll}
\hline \multicolumn{2}{l}{ LIOPHANT Officers } \\
\hline President & A.G. Bruzzone, agostino@ itim.unige.it \\
\hline Director & E. Bocca, enrico.bocca@liophant.org \\
\hline Secretary & A. Devoti, devoti.a@ @iveco.com \\
\hline Treasurer & M arina M asseimassei@ itim.unige.it \\
\hline Repr. EuRoSIm & A.G. Bruzzone, agostino@ itim.unige.it \\
\hline Deputy & F. Longo, f.longo@ unical.it \\
\hline Edit. Board SNE & F. Longo, f.longo@ unical.it \\
\hline Web EuRoSim & F. Longo, f.longo@ unical.it \\
\hline
\end{tabular}

\section{LSS - Latvian Simulation Society}

The Latvian Simulation Society (LSS) has been founded in 1990 as the first professional simulation organisation in the field of Modelling and simulation in the post-Soviet area. Its members represent the main simulation centres in Latvia, including both academic and industrial sectors.

\section{$\rightarrow$ briedis.itl.rtu.lv/imb/}

莑”= merkur@itl.rtu.lv

$\triangle$ LSS / Yuri Merkuryev, Dept. of Modelling and Simulation Riga Technical University Kalku street 1, Riga, LV-1658, LATVIA

\begin{tabular}{ll}
\hline LSS Officers & \\
\hline President & Yuri M erkuryev, merkur@itl.rtu.Iv \\
\hline Secretary & Artis Teilans, Artis.Teilans@ exigenservices.com \\
\hline Repr. EuROSIm & Yuri M erkuryev, merkur@ itl.rtu.Iv \\
\hline Deputy & Artis Teilans, Artis.Teilans@ @xigenservices.com \\
\hline Edit. Board SNE & Yuri M erkuryev, merkur@itl.rtu.Iv \\
\hline Web EuROSIM & Vitaly Bolshakov, vitalijs.bolsakovs@ rtu.Iv \\
\hline
\end{tabular}




\section{KA-SIM Kosovo Simulation Society}

Kosova Association for Modeling and Simulation (KASIM, founded in 2009), is part of Kosova Association of Control, Automation and Systems Engineering (KACASE). KA-CASE was registered in 2006 as non Profit Organization and since 2009 is National Member of IFAC - International Federation of Automatic Control. KA-SIM joined EUROSIM as Observer Member in 2011. In 2016, KA-SIM became full member.

KA-SIM has about 50 members, and is organizing the international conference series International Conference in Business, Technology and Innovation, in November, in Durrhes, Albania, and IFAC Simulation Workshops in Pristina.

$\rightarrow$ www.ubt-uni.net/ka-case

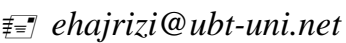

$\triangle$ MOD\&SIM KA-CASE; Att. Dr. Edmond Hajrizi Univ. for Business and Technology (UBT)

Lagjja Kalabria p.n., 10000 Prishtina, Kosovo

\begin{tabular}{ll}
\hline KA-SIM Officers \\
\hline President & Edmond Hajrizi, ehajrizi@ ubt-uni.net \\
\hline Vice president & M uzafer Shala, info@ ka-sim.com \\
\hline Secretary & Lulzim Beqiri, info@ ka-sim.com \\
\hline Treasurer & Selman Berisha, info@ ka-sim.com \\
\hline Repr. EuRoSim & Edmond Hajrizi, ehajrizi@ ubt-uni.net \\
\hline Deputy & M uzafer Shala, info@ ka-sim.com \\
\hline Edit. Board SNE & Edmond Hajrizi, ehajrizi@ ubt-uni.net \\
\hline Web EuRoSIm & Betim Gashi, info@ ka-sim.com \\
\hline
\end{tabular}

\section{PSCS - Polish Society for Computer Simulation}

PSCS was founded in 1993 in Warsaw. PSCS is a scientific, non-profit association of members from universities, research institutes and industry in Poland with common interests in variety of methods of computer simulations and its applications. At present PSCS counts 257 members.

$\rightarrow$ www.eurosim.info (www.ptsk.man.bialystok.pl)

奉”lleon@ibib.waw.pl

$\triangle$ PSCS / Leon Bobrowski, c/o IBIB PAN,

ul. Trojdena 4 (p.416), 02-109 Warszawa, Poland

\begin{tabular}{ll}
\hline PSCS Officers & \\
\hline President & Leon Bobrowski, leon@ibib.waw.pl \\
\hline Vice president & Tadeusz Nowicki, \\
& Tadeusz.Nowicki@wat.edu.pl \\
\hline Treasurer & Z. Sosnowski, zenon@ii.pb.bialystok.pl \\
\hline Secretary & Zdzislaw Galkowski, \\
& Zdzislaw.Galkowski@simr.pw.edu.pl \\
\hline Repr. EuRoSIm & Leon Bobrowski, leon@ibib.waw.pl \\
\hline Deputy & Tadeusz Nowicki, tadeusz.nowicki@wat.edu.pl \\
\hline Edit. Board SNE & Zenon Sosnowski, z.sosnowski@pb.ed.pl \\
\hline Web EuRoSIM & Magdalena Topczewska \\
& m.topczewska@ pb.edu.pl \\
\hline
\end{tabular}

\section{SIMS - Scandinavian Simulation Society}

SIMS is the Scandinavian Simulation Society with members from the four Nordic countries Denmark, Finland, Norway and Sweden. The SIMS history goes back to 1959. SIMS practical matters are taken care of by the SIMS board consisting of two representatives from each Nordic country (Iceland one board member).

SIMS Structure. SIMS is organised as federation of regional societies. There are FinSim (Finnish Simulation Forum), DKSIM (Dansk Simuleringsforening) and NFA (Norsk Forening for Automatisering).

$\rightarrow$ www.scansims.org

莑- esko.juuso@oulu.fi

$\triangle$ SIMS / Erik Dahlquist, School of Business, Society and Engineering, Department of Energy, Building and Environment, Mälardalen University, P.O.Box 883, 72123 Västerås, Sweden

\begin{tabular}{ll}
\hline SIM S Officers & \\
\hline President & Erik Dahlquist, erik.dahlquist@mdh.se \\
\hline Vice president & Bernd Lie, lie@ hit.noe \\
\hline Treasurer & $\begin{array}{l}\text { Vadim Engelson, } \\
\text { vadim.engelson@ mathcore.com }\end{array}$ \\
\hline Repr. EuRoSIM & Erik Dahlquist, erik.dahlquist@ mdh.se \\
\hline Edit. Board SNE & Esko Juuso, esko.juuso@oulu.fi \\
\hline Web EuRoSIm & Vadim Engelson, \\
& vadim.engelson@ mathcore.com
\end{tabular}




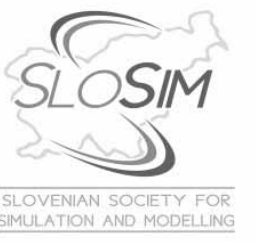

\section{SLOSIM - Slovenian Society for Simulation and Modelling}

SLOSIM - Slovenian Society for Simulation and Modelling was established in 1994 and became the full member of EUROSIM in 1996. Currently it has 90 members from both Slovenian universities, institutes, and industry. It promotes modelling and simulation approaches to problem solving in industrial as well as in academic environments by establishing communication and cooperation among corresponding teams.

$\rightarrow$ www.slosim.si

莑”slosim@fe.uni-lj.si

$\triangle$ SLOSIM / Vito Logar, Faculty of Electrical

Engineering, University of Ljubljana,

Tržaška 25, 1000 Ljubljana, Slovenia

\begin{tabular}{ll}
\hline \multicolumn{2}{l}{ SLOSIM Officers } \\
\hline President & Vito Logar, vito.logar@fe.uni-lj.si \\
\hline Vice president & Božidar Šarler, bozidar.sarler@ ung.si \\
\hline Secretary & Aleš Belič, ales.belic@ sandoz.com \\
\hline Treasurer & Milan Simčič, milan.simcic@fe.uni-lj.si \\
\hline Repr. EuRoSıM & B. Zupančič, borut.zupancic@fe.uni-lj.si \\
\hline Deputy & Vito Logar, vito.logar@fe.uni-lj.si \\
\hline Edit. Board SNE & B. Zupančič, borut.zupancic@fe.uni-lj.si \\
& Vito Logar, vito.logar@fe.uni-lj.si \\
& Blaž Rodič, blaz.rodic@fis.unm.si \\
\hline Web EuRoSım & Vito Logar, vito.logar@fe.uni-lj.si \\
\hline
\end{tabular}

\section{UKSIM - United Kingdom Simulation Society}

The UK Simulation Society is very active in organizing conferences, meetings and workshops. UKSim holds its annual conference in the March-April period. In recent years the conference has always been held at Emmanuel College, Cambridge. The Asia Modelling and Simulation Section (AMSS) of UKSim holds 4-5 conferences per year including the EMS (European Modelling Symposium), an event mainly aimed at young researchers, organized each year by UKSim in different European cities. Membership of the UK Simulation Society is free to participants of any of our conferences and their co-authors.

$\rightarrow$ www.uksim.org.uk

奉”david.al-dabass@ntu.ac.uk
UKSIM / Prof. David Al-Dabass

Computing \& Informatics,

Nottingham Trent University

Clifton lane, Nottingham, NG11 8NS

United Kingdom

\begin{tabular}{ll}
\hline UKSIM Officers & \\
\hline President & $\begin{array}{l}\text { David Al-Dabass, } \\
\text { david.al-dabass@ ntu.ac.uk }\end{array}$ \\
\hline Secretary & A. Orsoni, A.Orsoni@ @ingston.ac.uk \\
\hline Treasurer & A. Orsoni, A.Orsoni@kingston.ac.uk \\
\hline $\begin{array}{l}\text { Membership } \\
\text { chair }\end{array}$ & G. Jenkins, glenn.l.jenkins@ smu.ac.uk \\
\hline $\begin{array}{l}\text { Local/Venue } \\
\text { chair }\end{array}$ & Richard Cant, richard.cant@ ntu.ac.uk \\
\hline Repr. EuRoSIM & A. Orsoni, A.Orsoni@ kingston.ac.uk \\
\hline Deputy & G. Jenkins, glenn.l.jenkins@ smu.ac.uk \\
\hline Edit. Board SNE & A. Orsoni, A.Orsoni@ kingston.ac.uk \\
\hline
\end{tabular}

Last data update March 2016

\section{RNSS - Russian Simulation Society}

NSS - The Russian National Simulation Society (Национальное Общество Имитационного Моделирования - НОИМ) was officially registered in Russian Federation on February 11, 2011. In February 2012 NSS has been accepted as an observer member of EUROSIM, and in 2015 RNSS has become full member.

$\rightarrow$ www.simulation.su
麦” yusupov@iias.spb.su
RNSS / R.M. Yusupov

St. Petersburg Institute of Informatics and Automation RAS, 199178, St. Petersburg, 14th lin. V.O, 39

\begin{tabular}{ll}
\hline RNSS Officers & \\
\hline President & R. M. Yusupov, yusupov@iias.spb.su \\
\hline Chair Man. Board & A. Plotnikov, plotnikov@sstc.spb.ru \\
\hline Secretary & M. Dolmatov, dolmatov@ simulation.su \\
\hline Repr. EuRoSim & $\begin{array}{l}\text { R.M. Yusupov, yusupov@iias.spb.su } \\
\text { Y. Senichenkov, } \\
\text { senyb@dcn.icc.spbstu.ru }\end{array}$ \\
\hline Deputy & B. Sokolov, sokol@ iias.spb.su \\
\hline Edit. Board SNE & $\begin{array}{c}\text { Y. Senichenkov, } \\
\text { senyb@ dcn.icc.spbstu.ru }\end{array}$ \\
\hline
\end{tabular}
Last data update June 2016 


\section{EUROSIM OBSERVER M EMBERS}

\section{ROM SIM - Romanian Modelling and Simulation Society}

ROMSIM has been founded in 1990 as a non-profit society, devoted to theoretical and applied aspects of modelling and simulation of systems. ROMSIM currently has about 100 members from Romania and Moldavia.

$\rightarrow$ www.eurosim.info (www.ici.ro/romsim)

拝三sflorin@ici.ro

$\triangle$ ROMSIM / Florin Hartescu,

National Institute for Research in Informatics, Averescu Av. 8 - 10, 71316 Bucharest, Romania

\begin{tabular}{lr}
\hline ROMSIM Officers \\
\hline $\begin{array}{l}\text { President } \\
\text { Vice president }\end{array}$ & $\begin{array}{r}\text { Florin Hartescu, flory@ici.ro } \\
\text { Marius Radulescu, } \\
\text { mradulescu.csmro@yahoo.com }\end{array}$ \\
\hline Repr. EUROSIm & $\begin{array}{r}\text { Marius Radulescu, } \\
\text { mradulescu.csmro@yahoo.com }\end{array}$ \\
\hline Deputy & Florin Hartescu, flory@ici.ro \\
\hline Edit. Board SNE & Constanta Zoe Radulescu, zoe@ ici.ro \\
\hline Web EuRoSIM & Florin Hartescu, flory@ici.ro \\
\hline
\end{tabular}

\section{IMOS - Italian Modelling and Simulation Association}

MIMOS (Movimento Italiano Modellazione e Simulazione - Italian Modelling and Simulation Association) is the Italian association grouping companies, professionals, universities, and research institutions working in the field of modelling, simulation, virtual reality and 3D, with the aim of enhancing the culture of 'virtuality' in Italy, in every application area.

M IM OS became EUROSIM Observer Member in 2016 and is preparing application for full membership.

$\rightarrow w w w . m i m o s . i t$

麦”roma@mimos.it-info@mimos.it

$\triangle$ MIMOS - Movimento Italiano Modellazione e Simulazione; via Ugo Foscolo 4, 10126 Torino - via Laurentina 760, 00143 Roma

\begin{tabular}{ll}
\hline MIMOS Officers & \\
\hline President & Paolo Proietti, roma@mimos.it \\
\hline Secretary & Davide Borra, segreteria@ mimos.it \\
\hline Treasurer & Davide Borra, segreteria@mimos.it \\
\hline Repr. EuroSIM & Paolo Proietti, roma@ mimos.it \\
\hline Deputy & $\begin{array}{l}\text { Agostino Bruzzone, ago- } \\
\text { stino @ itim.unige.it }\end{array}$ \\
\hline Edit. Board SNE & Paolo Proietti, roma@ mimos.it \\
\hline & \multicolumn{1}{c}{ Last data update December 2016 }
\end{tabular}

\section{CANDIDATES}

\section{Albanian Simulation Society}

At the Department of Statistics and Applied Informatics, Faculty of Economy, University of Tirana, Prof. Dr. Kozeta Sevrani at present is setting up an Albanian Simulation Society. Kozeta Sevrani, professor of Computer Science and Management Information Systems, and head of the Department of Mathematics, Statistics and Applied Informatic, has attended a EUROSIM board meeting in Vienna and has presented simulation activities in Albania and the new simulation society.

The society - constitution and bylaws are being worked out - will be involved in different international and local simulation projects, and will be engaged in the organisation of the conference series ISTI - Information Systems and Technology. The society intends to become a EUROSIM Observer Member.

毒”kozeta.sevrani@unitir.edu.al

$\triangle$ Albanian Simulation Goup, attn. Kozeta Sevrani University of Tirana, Faculty of Economy rr. Elbasanit, Tirana 355 Albania

\begin{tabular}{lc}
$\begin{array}{l}\text { Albanian Simulation Society- Officers (Planned) } \\
\text { President }\end{array}$ & $\begin{array}{c}\text { Kozeta Sevrani, } \\
\text { kozeta.sevrani@unitir.edu.al }\end{array}$ \\
\hline Secretary & \\
\hline Treasurer & Kozeta Sevrani, \\
Repr. EuroSim & kozeta.sevrani@unitir.edu.al \\
\hline Edit. Board SNE & Albana Gorishti, \\
& albana.gorishti@unitir.edu.al \\
& Majlinda Godolja, \\
majlinda.godolja@fshn.edu.al \\
Last data update December 2016
\end{tabular}


EUROSIM

\section{Federation of European Simulation Societies}

General Information. EUROSIM, the Federation of European Simulation Societies, was set up in 1989. The purpose of EUROSIM is to provide a European forum for simulation societies and groups to promote advancement of modelling and simulation in industry, research, and development.

Member Societies. EUROSIM members may be national simulation societies and regional or international societies and groups dealing with modelling and simulation. At present EUROSIM has sixteen Full Members and two Observer Members, and one member candidate.

$\rightarrow$ www.eurosim.info

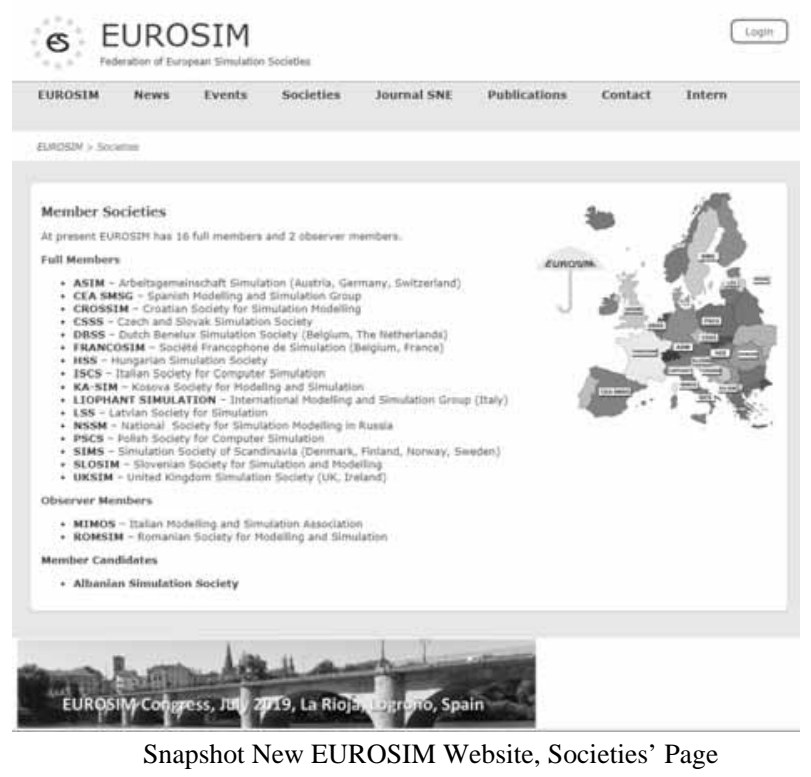

\section{EUROSIM Events}

EUROSIM is supporting, organising and co-sponsoring the following event types:

- EUROSIM Congress - triennial, organized by a EUROSIM member society

- EUROSIM Conference - international conference by a EUROSIM member society with EUROSIM advertising support

- EUROSIM Event - international conference or workshop organized by EUROSIM member society

- EUROSIM Co-Sponsorship - int. conferences of other organisations with mutual benefit contract
The EUROSIM Congress can be seen as constant within these developments - each three years simulationists from all over the world gather in one European country to exchange information on development in modelling and simulation. The $10^{\text {th }}$ EUROSIM congress will take place in July 2019 in La Rioja, Logroño, Spain.

EUROSIM 2019
10
th EUROSIM Congress on M odelling and Simulation
July 2019, La Rioja, Logroño, Spain
www.eurosim.info/events/eurosim-congress/

\section{EUROSIM Conferences}

EUROSIM member societies are entitled to organise a EUROSIM Conference - an international conference on any subject on modelling and simulation. An international conference organised under the trademark EUROSIM Conference is advertised via EUROSIM's mailing data base, and by publication space in SNE - Simulation Notes Europe.

Reduced fees are offered to all members of the EUROSIM member societies. EUROSIM expects a small percentage of the income.

At present the following conferences are organised under the trademark EUROSIM Conference:

- MATHMOD Conference Series, Vienna, Austria (organised by ASIM, the German simulation society), see www.mathmod.org

- $\quad$ ICBTI - UBT Conference Series, Durres, Albania (organized by KA-SIM, the simulation Society of Kosovo), see conferences.ubt-uni.net

\section{MATHMOD 2018}

$9^{\text {th }}$ Vienna Int. Conference on M athematical Modelling February 21 - 23, 2018, TU Wien, Vienna, Austria www.mathmod.at

The MATHMOD 2018 continues the conference series MATHMOD Vienna in February 2018. It provides a forum for professionals, researchers, and experts in the field of theoretic and applied aspects of mathematical modelling for systems of dynamic nature.

The scope of MATHMOD 2018 conference covers theoretic and applied aspects of the various types of mathematical modelling (equations of various types, automata, 
Petri nets, bond graphs, qualitative and fuzzy models, etc.) for systems of dynamic nature (deterministic, stochastic, continuous, discrete or hybrid with respect to time, etc.). The topics to be discussed include e.g.

- modelling theory; first-principles, identification, optimization, order reduction and validation

- $\quad$ automation of modelling and software tools

- computer modelling, modelling for/by simulation, cosimulation, modelling standards

- $\quad$ qualitative, modular, interdisciplinary modelling

- comparison of methods for modelling, alternative modelling methods (CAS, fuzzy, NN, QSS, etc.)

- model analysis and calibration, effects of modelling errors on overall performance of an engineering system

- $\quad$ applications in the field of engineering systems and in natural sciences

- applications in environmental systems, biotechnology, etc.

- applications in operation research, logistics and planning

- $\quad$ applications in medicine, physiology, health care and health technology assessment

- education in/for/with modelling

- modelling aspects in scientific computing

- modelling for control and real-time applications

\section{EUROSIM Conference Co-operation}

A special co-operation exists with the I3M Conference Series, organised by LIOPHANT and co-spomsoerd by MIMOS (EUROSIM societies). Every year, the I3M M ulticonference renovates the opportunity to bring together researchers, scientists and practitioners, from the Mediterranean Area, Latin \& North Americas, Europe, Asia, Africa and Australia, who are concerned with Modeling and Simulation in Industry and Academia. I3M part are:

- MAS - Conf. on Modelling \& Applied Simulation

- EMSS - European Modelling \& Simulation Symposium

- NSP - New Simulation Project Workshop

- HMS - Conference on Harbour, Maritime \& Multimodal Logistics M\&S - HMS

- IMAACA - Modelling \& Simulation Network Workshop

- DHSS - Int. WS Defense and Homeland Security Sim.

- IWISH - Int. WS on Innovative Simulation for Health Care

- SESDE - Int. WS on Simulation for Energy, Sustainable Development \& Environment

- FoodOPS- Int. WS on Food Operations \& Processing Sim.

\section{I3M 2018}

INTERNATIONAL M ULTIDISCIPLINARY M ODEUNG \& SIMULATION MULTICONFERENCE

September 2018, 2018, Budapest, Hungary www.liophant.org/i3m/

\section{New EUROSIM Website}

The EUROSIM website www.eurosim.info has been completely redesigned and installed on state-of-the-art technology. Responsive design adapts the layout to a variety of devices. Most essential and recent content has been transferred to the new site. We apologize for the problems with the previous website in begin of 2017 , caused by severe hacking attacks.

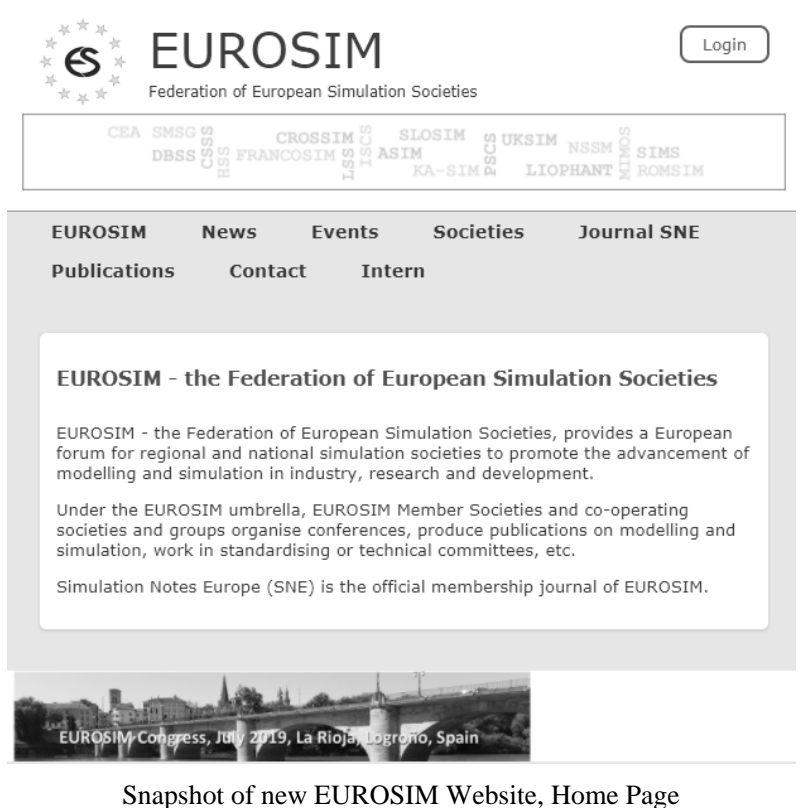

A short guide through the sections should explain the website's features (snapshot of homepage see before).

The News section and the Events section are maintained by the EUROSIM webmaster(s) compiling classical news lists and events lists. Please send an e-mail to webmaster@eurosim.info if you have an announcement to be posted (general news, general news about a society, conference announcements).

The Societies section - see snapshot of societies' webpage before - provides information on all EUROSIM societies - the content is managed by the societies themselves, usually structured with first page, and with sections on news, events, reports, and publications.

The first webpage of each society gives a summary on the society, and lists officers of the society. We ask all societies to update this list regularly, with actual president and other administrative officers and with officers responsible for society representation in EUROSIM, for SNE news editorial board, and for the society's content maintenance at EUROSIM website -snapshot next page. 


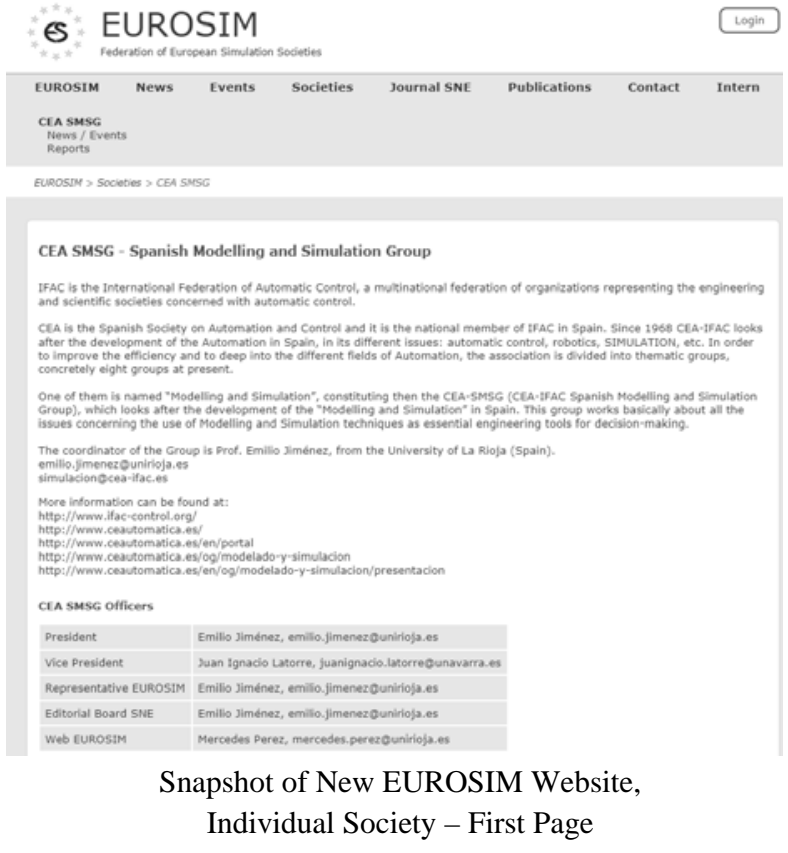

The Journal SNE section shortly introduces SNE - Simulation Notes Europe as official membership journal of EUROSIM and links to the SNE website. With Online SNE (Online ISSN 2306-0271) the publisher ARGESIM follows the Open Access strategy, allowing download of published contributions for free.

Access for members of EUROSIM Societies. High-resolution Online SNE, full SNE Archive, and sources of benchmarks or other additional documents are available for members of EUROSIM societies. Login data are distributed by the EUROSIM societies to their members directly, with yearly change of password:

- (Sample) Login: asim-member

- Password: $\mathbf{x x x x x \mathbf { x }}$

This login works as well at the SNE website (for SNE download; snapshots next pages), as well as at the EUROSIM website (for publication download, see below) please use Login button, or enter Login section resp.

There is also available a print version of SNE. The publisher ARGESIM itself produces sample copies for promotional purposes (samples for authors and societies, copies for conferences, etc.). And generally, TU-Verlag (TU Wien Publisher - www.tuverlag.at) provides Printon-Demand service for SNE issues, whereby each Print SNE issue is identified by an individual ISBN number from TU Verlag - starting with SNE Volume 27. Printed copies can simply be ordered in the webstore at www.tuverlag.at.
The Publications section is intended to provide Proceedings from EUROSIM congresses and from EUROSIM conferences (e.g. MATHMOD, ICBTI). Members of EUROSIM societies can download full documents using the Login described before - starting in October 2017.

And last but not least the Contact section provides main contact emails for EUROSIM and for all member societies - sample see below (update taken from the individual society pages).

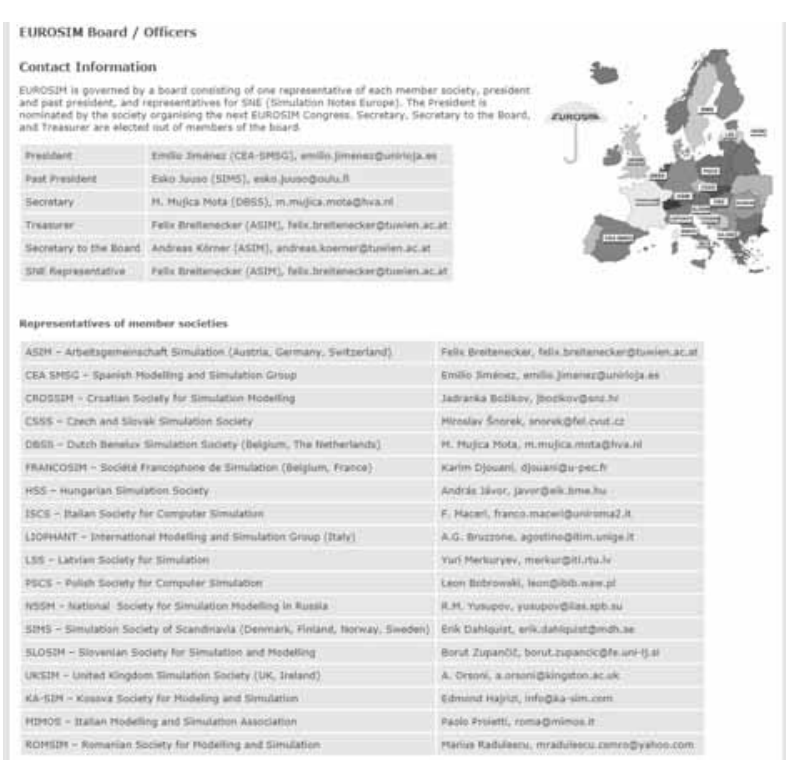

Snapshot of New EUROSIM Website, Contact Info Page

Access for Board members. The EUROSIM Board consists of represenatives from all member societies (one per member society). Each representative has frontend access to the section Internal with documents of board meetings, and EUROSIM bylaws, and other internal documents.

Furthermore, each society representative has personal editing access to the backend of the EUROSIM website in order to maintain information on the specific content of the Societies section.

If you have any questions or comments please send an e-mail towebmaster@eurosim.info

or towebmaster@sne-journal.info 


\section{New SNE Website}

Thew relaunch of the website for the journal SNE Simulation Notes Europe is completed. Members of EUROSIM societies can download high-resolution SNE issues and SNE single contributions, and further publications. Login data are distributed by the EUROSIM societies to their members directly, with yearly change of password:

- (Sample) Login: asim-member

- Password: xxxxxx

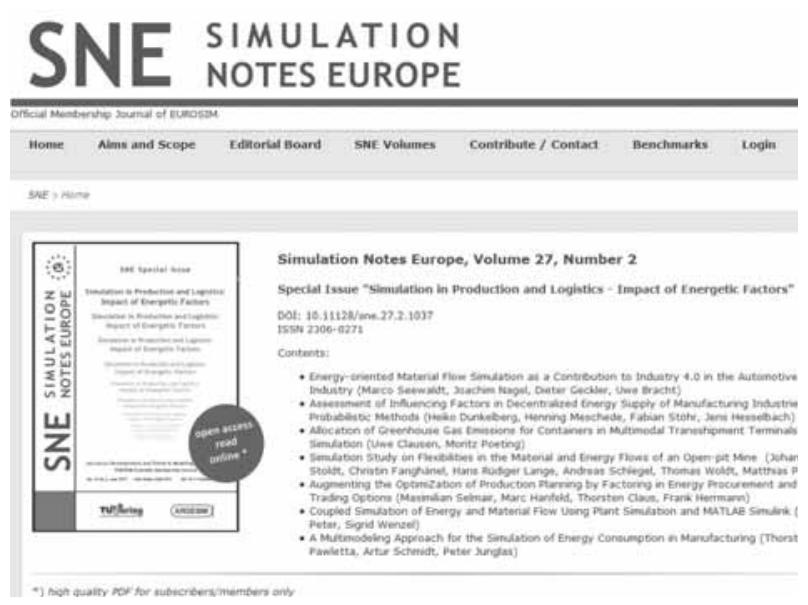

New SNE Website, Homepage

Volume 26
SNE 26(1) Articles
SNE 26(2) Articles
SNE 26(3) Articles
SNE 26(4) Articles
SNE $>$ SNE Volumes $>$ Volume 26

Simulation Notes Europe, Volume 26, 2016

SNE 26(1), March 2016

ARGESIM / EUROSIM

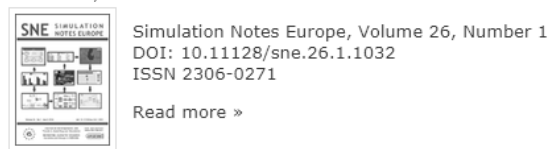

SNE 26(2), June 2016

ARGESIM / EUROSIM

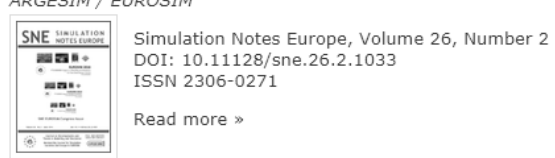

SNE 26(3), Special Issue "System Dynamics", September 2016 ARGESIM / EUROSIM

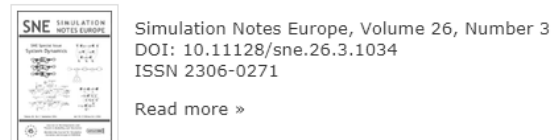

New SNE Website, SNE Volumes Page
The entry page of SNE's new website (snapshot left, above) offers a direct quick access to the newest issue, and the menu with sections first provides information on aims and scope, and on the editorial board.

The sections SNE Volumes allows selection and display of the issues of the specific volume, see snapshot left, below. There, the Read more link in the issue display opens information and download possibilities for the specific issue. Alternatively, the SNE nn(n) Articles link at top of the volumes page switches to the page with list of articles in this issue - snapshot below.

SIMULATION
NOTES EUROPE
Official Membership Journal of EUROSIM
Home Aims and Scope Editorial Board SNE Volumes
Contribute / Contact Login
Volume 26 Articles
SNE 26(1) Artes
SNE 26(2) Articles
SNE 26(3) Articles
SNE 26(4) Articles

$$
\text { SNE }>\text { SNE Volumes > Volume } 26>\text { SNE 26(1) Articles }
$$

Simulation Notes Europe, Volume 26(1), March 2016

Modeling Garage Parking

Oliver Ullich | Charaf Azzouzi | Benjamin Brandt | Daniel Lückerath | Naphtali Rishe

Simulation Notes Europe SNE 26(1), 2016, 1-8 DOI: $10.11128 /$ sne.26.tn. 10321

Read more »

Human Activity Pattern Recognition based on Continuous Data from a Body Worn Sensor placed on the Hand Wrist using Hidden Markov Models

Sarah Fallmann | Johannes Kropf

Simulation Notes Europe SNE 26(1), 2016, 9-16 DOI: $10.11128 /$ sne.26.tn. 10322

Read more »

Automatic IO Device Selection for Ambient Environments Brigitte Kupka | Jonathan Steinhart | Miroslav Sili | Matthias Gira | Christopher Mayer

SNE Simulation Notes Europe SNE 26(1), 2016, 17-22 DOI: $10.11128 /$ sne $26.5 W .10323$

Read more »

New SNE Website, Articles List Page

And there finally, the Read more link in the articles list page provides bibliographical information, abstract, and download possibilities for the specific article - see next snapshot. Here, at bottom, download files for the article are offered. The pdf-files with ending OA are open access, download of files with ending RA (restricted access) requires Login with the society's member login. 
In case of benchmark articles here also files with sources are offered for download with restricted access, or other specific documents related with the article

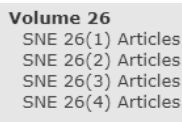

Simulation Notes Europe, Volume 26(1), March 2016

Modeling Garage Parking

Oliver Ullrich | Charaf Azzouzi | Benjamin Brandt | Daniel Lückerath |

Simulation Notes Europe SNE 26(1), 2016, 1-8 DOI: $10.11128 /$ sne.26.tn.1032

Abstract

Described is a simulation model of cruising for garage parking, intended both for the calibration and evaluation of real-time parking recommendation methods, and as a base for predictive guidance to available parking. The model combines the event-based and agent-based simulation approaches to represent the parking garage and the driver behavior. It is validated by simulating a real-world parking garage and comparing the model's output with garage's state over the course of an operational day, even though specific results are not yet precise enough for the intended use.

After an introduction to scope and aims, the paper shares some background on garage parking and related work, followed by a description of the simulation model, and its validation based on a representation of a realworld parking garage.

Related Files

- sne.26.1.10321.tn.OA.pdf $205 \mathrm{~KB}$

- sne.26.1.10321.tn.RA.pdf 5 MB

New SNE Webpage, SNE Article Page

The sections Contribute / Contact provide information for authors, and submission templates in doc format and tex format can be downloaded, as well as information on the reviewing process.

And last but not least, the section Benchmarks links to definition and solutions of the ARGESIM Benchmarks for Modelling Approaches and Simulation Implementations. Definitions and solution are published in SNE as Benchmark Solution (2 p. SNE), Benchmark Report (4 to 6 p. SNE), or Benchmark Study (6 to 10 p. SNE)

Since 2012, Online SNE Issues and Online SNE Articles are identified by a DOI (Digital Object Identifier) and registered at Crossref immediately after publication. DOI prefix is 10.11128 , followed by issue or article identification (this issue DOI 101128/sne.27.4.1039). The relaunch of the SNE webserver required modification of DOI registration at Crossref, but search and access via DOI is working again since October 2017.

If you have any questions or comments please send an e-mail towebmaster@eurosim.info or towebmaster@sne-journal.info

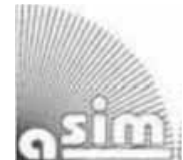

\section{ASIM \\ German Simulation Society Arbeitsgemeinschaft Simulation}

ASIM (Arbeitsgemeinschaft Simulation) is the association for simulation in the German speaking area, servicing mainly Germany, Switzerland and Austria. ASIM was founded in 1981 and has now about 600 individual members, and 90 institutional or industrial members.

$\rightarrow$ www.asim-gi.org with members' area

莑”info@asim-gi.org,admin@asim-gi.org

$\triangle$ ASIM - Inst. f. Analysis and Scientific Computing TU Wien - Technical Univ. Vienna Wiedner Hauptstrasse 8-10, 1040 Vienna, Austria

ASIM is organising / co-organising the following international conferences:

- ASIM Int. Conference 'Simulation in Production and Logistics' - biannual - ASIM SPL Conference

- ASIM 'Symposium Simulation Techniques' - biannual - ASIM SST Conference

- MATHMOD Int. Vienna Conference on Mathematical Modelling - triennial

Furthermore, ASIM is co-sponsor of WSC - Winter Simulation Conference, anf of I3M.

ASIM is structured in ASIM Sections, dealing with various methods and applications. Within the sections or across sections, working groups discuss and document new developments

\section{Coming Events}

Every two years the ASIM Symposium Simulation Technique' ASIM SST is organized.

ASIM SST 2018
SYM POSIUM SIMULATION TECHNIQUES
October 4 - 5, 2018, HCU Hamburg, Germany
www.asim2018.de

Aims and Scope. The conference covers methods and applications in modelling and simulation. The topics are:

- Mathematical methods

- Methods from Computer science and computer engineering

- Applications in various areas

Please contact the website for further details: www. asim2018.de 


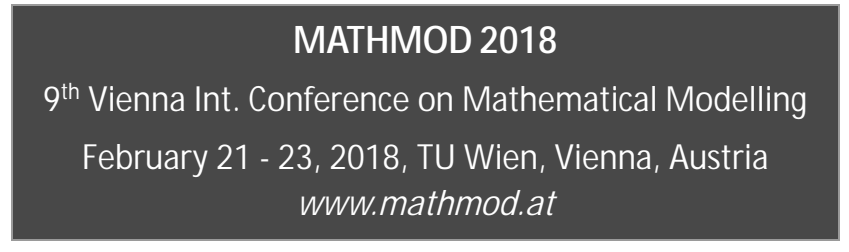

M ATHM OD 2018 provides a forum for professionals, researchers, and experts in the field of theoretic and applied aspects of mathematical modelling for systems of dynamic nature. ASIM is the main co-organiser of the MATHMOD conference series (EUROSIM Conference; see also news page $\mathrm{N} 9$ or www.mathmod.at.

SNE Promotion with Printed Issue. MATHMOD 2018 conference promotes SNE by providing for each participant a print copy of SNE 27(4), with a broad variety of contributions to modelling and simulation.

\section{New ASIM Website}

The ASIM website www.asim-gi.org has been completely redesigned and installed on state-of-the-art technology (snapshot of homepage see below). Responsive design adapts the layout to a variety of devices.

The webpage offers open access for basic versions of publications (proceedings, reports). ASIM members can download high-resolution publication, and proceedings, books, and reports with restricted access using their Personal ASIM Member Login; this login works also for SNE download at the SNE website and publication download at EUROSIM website.

ASIM, A. Körner, andreas.koerner@tuwien.ac.at

\section{CEA-SM SG - Spanish Modelling and Simulation Group}

CEA is the Spanish Society on Automation and Control and it is the national member of IFAC (International Federation of Automatic Control) in Spain. Since 1968 CEAIFAC looks after the development of the Automation in Spain, in its different issues: automatic control, robotics, SIMULATION, etc. The association is divided into national thematic groups, one of which is centered on Modeling, Simulation and Optimization, constituting the CEA Spanish Modeling and Simulation Group (CEA-SMSG). It looks after the development of the Modelling and Simulation (M\&S) in Spain, working basically on all the issues concerning the use of $M \& S$ techniques as essential engineering tools for decision-making and optimization. $\rightarrow$ http://www.ceautomatica.es/grupos/

$\rightarrow$ emilio.jimenez@unirioja.es

simulacion@cea-ifac.es

$\triangle$ CEA-SMSG / Emilio Jiménez, Department of Electrical Engineering, University of La Rioja, San José de Calasanz 31, 26004 Logroño (La Rioja), SPAIN

CEA SMSG Activities. The main usual activities of the group can be summarized as an annual meeting about modelling and simulation, inside CEA meeting on automation, a biannual symposium on Modeling and Simulation since 2012, specialized courses, a distribution list, a periodic elec-tronic report, technical books, a journal ('Revista Iberoamericana de Automática e Informática Industrial', translated as 'Latin American Journal of Automation and Industrial Computing' https://polipapers.upv.es/index.php/RIAI, indexed in the Journal Citation Reports JCR of Thomson Reuters), a trade agreement with Pearson Inc. for a collection of books, an award for the scientific contribution in automation and a specific award for modelling and simulation, sponsorship of events, etc.

In 2019 CEA SMSG will organize the international EUROSIM Congress in July 2019 in La Rioja, Logroño, Spain.

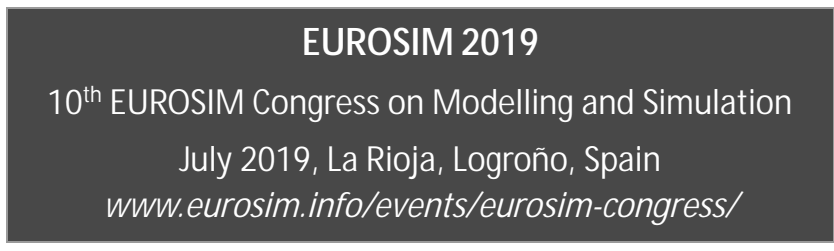

CEA SM SG Recent Events. As most interesting recent activities could be selected:

- I3M2017 - 12th International Mediterranean and Latin American Modelling Multiconference, 2017 http://www.msc-les.org/conf/i3m2017/, in Barcelona (Spain) September 2017, a multiconference.

- 38th annual meeting, inside the National Automat-ic Workshop (XXXV Jornadas de Automática) by the CEA-IFAC. September 2017, Gijón (Spain), which included the annual meeting of the CEA-SMSG (CEA-IFAC Spanish Modeling and Simula-tion Group), http://ja2017.es/

- IV Symposium CEA of Modeling Simulation and Optimization http://albergueweb1.uva.es/smso2018 organized by the CEA-SMSG in Valladolid (Spain) in January 2017.

Emilio Jiménez, emilio.jimenez@unirioja.es 


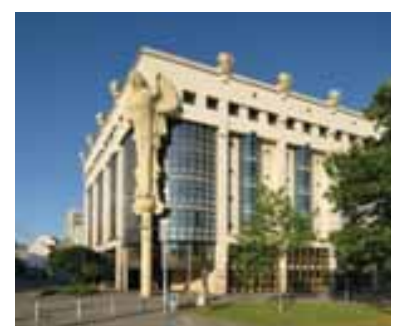

\section{T- MATHMOD 2018 Vienna \\ 9th Vienna International Conference on Mathematical Modelling February $21-23,2018$ at TU Wien}

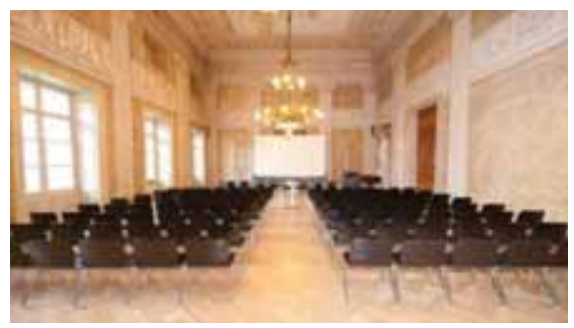

\section{SCOPE}

The scope of MATHMOD 2018 conference covers theoretic and applied aspects of various types of mathematical modelling (equations of various types, automata, Petri nets, bond graphs, qualitative and fuzzy models, etc.) for systems of dynamic nature (deterministic, stochastic, continuous, discrete or hybrid with respect to time, etc.).

The topics to be discussed include e.g.

- modelling theory; first-principles, identification, optimization, order reduction and validation

- automation of modelling and software tools

- computer modelling, modelling for/by simulation, co-simulation, modelling standards

- qualitative, modular, interdisciplinary modelling

- comparison of methods for modelling, alternative modelling methods (CAS, fuzzy, NN, QSS, etc.)

- model analysis and calibration, effects of modelling errors on overall performance of engineering systems

- applications in the field of engineering systems and in natural sciences

- applications in environmental systems, biotechnology, etc.

- applications in operation research, logistics and planning

- applications in medicine, physiology, health care and health systems

- education in/for/with modelling

- modelling aspects in scientific computing

- modelling for control and real-time applications

- machine learning, data analytics, big data

\section{SCIENTIFIC PROGRAMME}

- Sessions with Invited Plenary Contributions

- Thematic session with Full Contributions (oral presentations)

- Minisymposia with Full Contributions and Discussion Contributions (oral presentation)

- Short Presentation Sessions and Poster Display with Discussion Contributions and Student Contributions

- Tutorials / Exhibition for Methods, Tools and Software for Modelling and Simulation

\section{SOCIAL PROGRAMM}

The Social Programme includes Welcome Party, Cocktail Reception, Heurigen Evening, Art Exhibition, Café Simulation and a Programme for Accompanying Persons includes all social events and guided tours in Vienna.

\section{CONFERENCE CONTRIBUTIONS}

MATHMOD 2018 invites to submit contributions of following types:

- MATHMOD Full Contribution - full paper review; publication in IFAC-PapersOnLine and in Preprint Volume; oral presentation in thematic sessions or minisymposium

- MATHMOD Discussion Contribution - abstract review; publication in MATHMOD Extended Abstract Volume and in Preprint Volume; poster display with short oral presentation or oral presentation in minisymposium

- MATHMOD Student Contribution - abstract review; publication in MATHMOD Extended Abstract Volume and in Preprint Volume; poster display with short oral presentation

\section{Deadlines Full Contributions}

Submission Full Contribution Notification of Acceptance

Full Paper \& Preprint Abstract
Oct. 9, 2017

Jan. 12, 2018

Jan. 26, 2018

\section{Deadlines Discussion / Student Contributions}

$\begin{array}{ll}\text { Submission Abstract } & \text { Nov. 5, 2017 } \\ \text { Notification of Acceptance } & \text { Jan. 12, 2018 } \\ \text { Preprint Abstract } & \text { Jan. 26, 2018 }\end{array}$

\section{CONFERENCE PUBLICATIONS}

The MATHMOD Conference Proceedings are published at IFAC-PapersOnLine (Full Contributions and Invited Contributions with single DOIs). Discussion Contributions and Student Contributions (2-page estended abstract) are published in MATHMOD 2015 Extended Abstract Volume (Online) with ISBN and with Volume DOI. A MATHMOD 2015 Preprint Volument Volume with all contribution types will be available at the conference for participants (electronic version).

TU Wien / Inst. 376 Email: info@mathmod.at

further Information: 1040 Vienna, AustriaPhone/Fax: +43158801-37615/910111 www.mathmod.at 

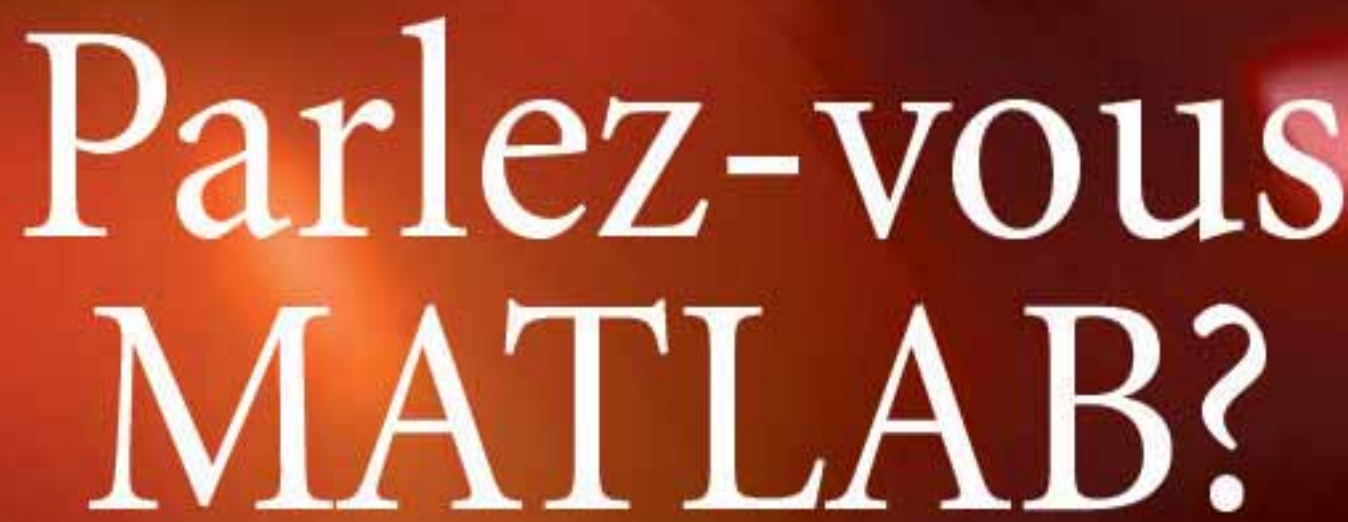

Ober eine Million Menschen wel twe it sprechen MATLAB. Ingenieure und Wissenschaftler in allen Bereichen - von der Luft- und Raumfahit tuber die Halbleiterindustrie bis zur Biotechnologie, Finanzdienstleistungen und Geo- und Mereswissenschuften - mutzen MATLAB, um ihre Ideen auszudrüdken. Sprechen Sie MATLAB?

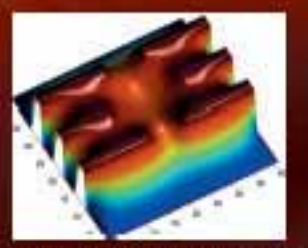

Modelliernug cines eloktrischen Potentials in cinem Qunumam Dot.

Dieses Reispiel finden Sie untern mwe mathworks dellic

The language of technical computing

w w w t tuverlag. at

ISBN 978-3-903024-62-5

||||||||||||||||||||||||||||||||||||||||| 